Göttinger Studien zur Entwicklungsökonomik Göttingen Studies in Development Economics

Herausgegeben von/Edited by Hermann Sautter und/and Stephan Klasen

Bd./Vol. 26
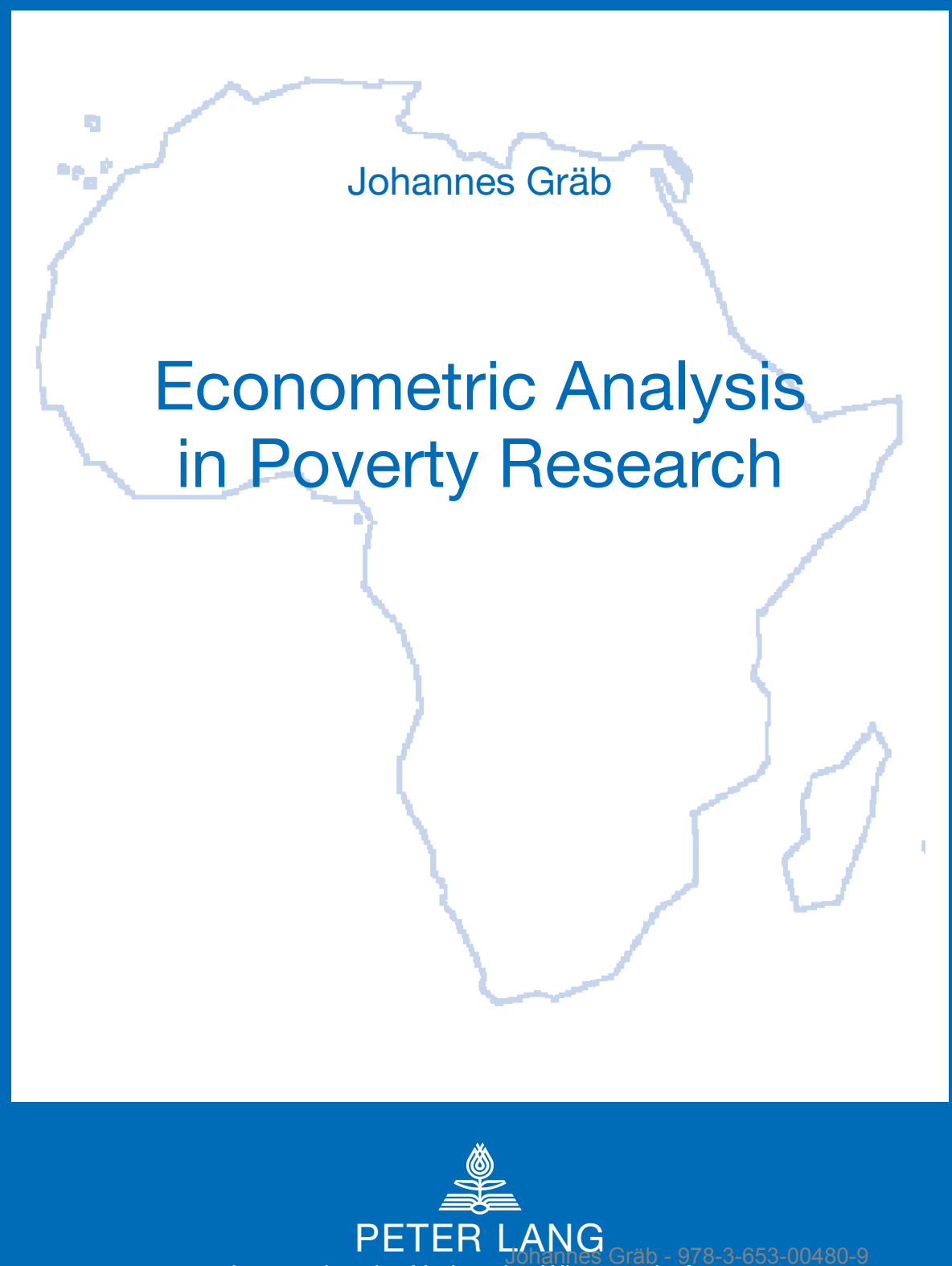

Internationaler Verlag der Wissenschaften 
Poverty and inequality persist in many regions of the developing world. This may be due mainly to an ineffective targeting of policies to address the root causes of poverty. Sustainable policy interventions are in need of reliable concepts of poverty and of a thorough understanding of the underlying mechanism that lead to such deprivation. The three essays of this book add to the debate concerning appropriate statistical tools in empirical development economics. The work proposes specific methodologies to analyze the extent of poverty and its underlying factors based on recent household surveys. The first chapter deals with a concept of poverty comparisons when panel data is at hand. The second chapter studies the determinants of spatial inequality using multilevel modelling. The third chapter analyzes the relation between a child's nutritional status and its survival probability.

Johannes Gräb, born in Göttingen in 1980, studied economics at the University of Mannheim. He was a Ph.D. Candidate as well as a research and teaching associate at the Department of Economics and the Center for Statistics at the University of Göttingen. He has also worked as a consultant for several international development agencies in various countries in Africa. 
Econometric Analysis in Poverty Research

Johannes Gräb - 978-3-653-00480-9

Downloaded from PubFactory at 01/11/2019 02:31:01AM

via free access 


\section{Göttinger Studien zur Entwicklungsökonomik Göttingen Studies in Development Economics}

Herausgegeben von/Edited by Hermann Sautter und/and Stephan Klasen

Bd./Vol. 26

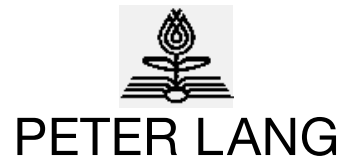

Frankfurt am Main · Berlin · Bern · Bruxelles · New York · Oxford · Wien 
Johannes Gräb

\title{
Econometric Analysis in Poverty Research
}

\author{
With Case Studies \\ from Developing Countries
}

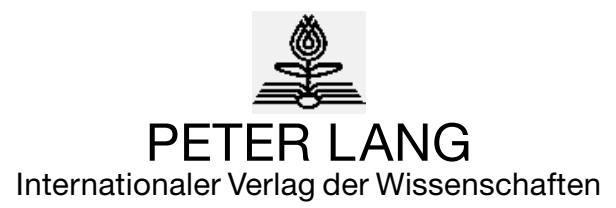

Johannes Gräb - 978-3-653-00480-9 


\title{
Bibliografische Information der Deutschen Nationalbibliothek
}

The Deutsche Nationalbibliothek lists this publication in the Deutsche Nationalbibliografie; detailed bibliographic data is available in the internet at http://dnb.d-nb.de.

Open Access: Die Online-Version dieser Publikation ist unter der internationalen Creative Commons Lizenz CC-BY 4.0 auf www.peterlang.com und www.econstor.eu veröffentlicht. Erfahren Sie mehr dazu, wie Sie dieses Werk nutzen können: http://creativecommons.org/licenses/by/4.0.

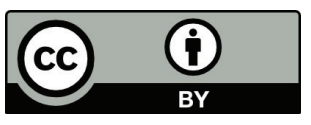

Das Werk enthält möglicherweise Inhalte, die von Drittanbietern lizensiert sind. Bei einer Wiederverwendung dieser Inhalte muss die Genehmigung des jeweiligen Drittanbieters eingeholt werden.

Dieses Buch ist Open Access verfügbar aufgrund der freundlichen Unterstützung des ZBW - Leibniz-Informationszentrum Wirtschaft.

\author{
D 7 \\ ISSN 1439-3395 \\ Isbn 978-3-653-00480-9 \\ (C) Peter Lang GmbH \\ Internationaler Verlag der Wissenschaften \\ Frankfurt am Main 2009 \\ www.peterlang.de
}




\section{Meinen Eltern}


Johannes Gräb - 978-3-653-00480-9

Downloaded from PubFactory at 01/11/2019 02:31:01AM

via free access 


\section{Editor's Preface}

With the declaration of the Millennium Development Goals in 2000, the global fight against poverty has become one of the central concerns in global policy debates. Poverty alleviation policies aim at reducing extreme poverty, hunger, poor health and education outcomes. Despite considerable efforts, many regions and countries in the world are still performing very badly concerning these dimensions of poverty. This may be due mainly to an ineffective targeting of policies to address the root causes of poverty. Sustainable policy interventions are in need of reliable concepts of poverty and of a thorough understanding of the underlying mechanism that lead to such deprivation.

This dissertation contributes to analyzing unresolved important issues in the fight against poverty by proposing and applying specific statistical methodologies to analyze the extent of poverty and its underlying factors based on recent household surveys in developing countries. In the first essay, Johannes Gräb is concerned with poverty measurement. In particular, the author elaborates a concept for poverty comparisons when the indicator of well being is observed over several points in time. Gräb shows that comparisons based upon the stochastic dominance methodology are not only robust to any specific poverty index and to any arbitrary setting of the poverty line but additionally to any aggregation procedures of individual incomes over time. He illustrates his approach by performing multiperiod poverty comparisons for Indonesia and Peru. The results show considerable dependence of poverty orderings on the aggregation procedures of income over time, emphasizing the relevance of the approach.

In the second essay Gräb takes a closer look at the importance of geographic factors in explaining observed spatial variation in household income. The author suggests a novel methodology to addresses this issue. He builds a multilevel random coefficient model able to decompose the variance in living standards across different spatial levels. Such an approach is particularly interesting from a political point of view since it allows effective targeting of spatial units. In the empirical part, Gräb decomposes the sources of spatial disparities in incomes among households in Burkina Faso showing that spatial disparities are not only driven by 
the spatial concentration of households with particular endowments but to a large extent also by disparities in community endowments.

The third essay takes into account the multidimensionality of poverty. Households in developing countries do not only suffer from low levels of income but also from other dimensions of well-being, such as health or education. It is therefore important to analyze the underling mechanism that lead to observed outcomes in non-monetary indicators of poverty to deduce effective poverty reduction policies. Gräb investigates the role of cultural, geographic, and political factors on the relationship of anthropometric outcomes of children and Under-5 mortality rates. He focuses on the unique situation of the territory around Lake Victoria which shows a pattern of low levels of malnutrition together with dramatically high rates of mortality found in no other region in Sub-Saharan Africa. Applying linear and nonlinear multilevel regression analysis the author finds a unique interplay of cultural, geographic and political factors in the Lake Victoria region to be responsible for causing the described paradox.

Johannes Gräb thus addresses a number of highly topical issues discussed in the ongoing literature on poverty and inequality in developing countries. He also provides very important new insights for our understanding of poverty in its many dimensions. Beyond, Gräb narrows the gap between the comprehensive statistical toolbox and its still limited application in development economic research. With his analysis, Gräb provides a valuable contribution to the economics literature on the empirical analysis of poverty in general, and on poverty comparisons, spatial income inequality, child mortality and undernutrition in particular.

Prof. Stephan Klasen, Ph.D.

Göttingen, June 2009 


\section{Author's Preface}

The submission of thesis dissertation marks the end of a long, eventful and challenging journey. I would like to acknowledge the many people who accompanied me along the way. Their great support made this dissertation possible and it is because of them that my graduate experience has been one that I will cherish forever.

First and foremost, I would like to express my sincere gratitude to my advisor Professor Michael Grimm. He provided me with many ideas and motivated me throughout the last years, and he gave me the freedom to pursue my academic work independently. His guidance, his patience and his willingness to help provided an excellent basis for this thesis. I could not have imagined having a better advisor and mentor for my Ph.D study.

I am also very grateful to Professor Stephan Klasen, who has been abundantly helpful, and has assisted me in numerous ways throughout this work. His detailed and constructive comments have been of great value for me.

I also wish to express my sincere thanks to Professor Stefan Sperlich for his detailed review and his excellent guidance in statistical analysis.

During this work I have collaborated with many colleagues at the University of Göttingen, and I wish to extend my warmest thanks to all those who have helped me with my work. In particular, I would like to thank my coauthor Jan Priebe for the great time we had studying the mysteries of Lake Victoria.

My deepest gratitude goes to my family for their unflagging support throughout my life. None of this would have been possible without the encouragement and understanding of my parents.

Finally, I am deeply thankful to my life partner, Johanna Bätzing, who has been a constant source of love, support and strength throughout this entire journey. Without her I would have struggled to find the inspiration and motivation needed to complete this dissertation.

Johannes Gräb

Bad Homburg, June 2009 
Johannes Gräb - 978-3-653-00480-9

Downloaded from PubFactory at 01/11/2019 02:31:01AM

via free access 


\section{Contents}

List of Tables $\quad$ xiv

List of Figures $\quad$ Xv

$\begin{array}{ll}\text { List of Abbreviations } & \text { xvii }\end{array}$

$\begin{array}{ll}\text { Introduction and Overview } & 1\end{array}$

1 Robust Multiperiod Poverty Comparisons 9

1.1 Introduction . . . . . . . . . . . . . . . . 10

1.2 Methodology ..................... 11

1.2.1 Stochastic dominance in a one-period welfare measure . . 11

1.2.2 Stochastic dominance in a two-period welfare measure . . 13

1.2.3 Stochastic dominance in a $n$-period welfare measure . . . 17

1.2.4 Relative poverty comparison . . . . . . . . . . 18

1.2.5 Estimation and inference . . . . . . . . . . . . 18

1.2.6 Bounds to multidimensional dominance . . . . . . . . . 19

1.3 Empirical illustration . . . . . . . . . . . . . . . . . . 19

1.3.1 Data . . . . . . . . . . . . . . . 19

1.3.2 Robust multiperiod poverty comparisons for the two- period case . . . . . . . . . . . . . 20

1.3.3 Robust multiperiod relative poverty comparisons for the two-period case within and across countries . . . . . . . 27

1.3.4 Robust multiperiod poverty comparisons for the $n$-period case .................... 30

1.4 Discussion . . . . . . . . . . . . . . . . . 31

2 Spatial inequalities explained - Evidence from Burkina Faso 33

2.1 Introduction . . . . . . . . . . . . . . . . . 34

2.2 Regional Growth and Inequality in Burkina Faso . . . . . . . . 36

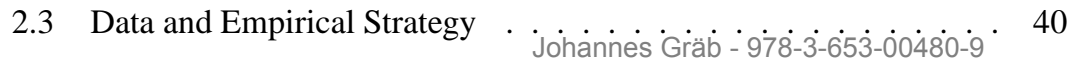


2.3.1 Data . . . . . . . . . . . . . . . 40

2.3.2 Empirical Strategy . . . . . . . . . . . . . . . . . . . 44

2.4 Results: Sources of Spatial Inequality . . . . . . . . . . . . . . 49

2.4.1 Model M0: The null model . . . . . . . . . . . . . . . . . 49

2.4.2 Model M1: The role of household characteristics . . . . 55

2.4.3 Model M2: The role of community characteristics . . . . 57

2.4.4 Model M3: The role of provincial and regional characteristics . . . . . . . . . . . . . . . . . . 59

2.4.5 Model M4: Variations in household level effects across communities . . . . . . . . . . . . 61

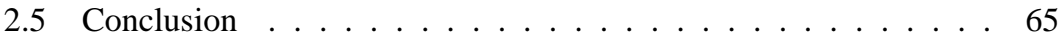

3 Low Malnutrition but High Mortality: Explaining the Paradox of the $\begin{array}{ll}\text { Lake Victoria Region } & 67\end{array}$

3.1 Introduction . . . . . . . . . . . . . . . . 68

3.2 The Paradox . . . . . . . . . . . . . . . . . 71

3.3 Theoretical Framework . . . . . . . . . . . . . . . 75

3.4 Geography vs. Ethnicity: The Kenyan Context . . . . . . . . . . 78

3.4.1 Nutritional Environment . . . . . . . . . . . . . . 78

3.4 .2 Epidemiological Factors . . . . . . . . . . . . . 81

3.4 .3 Cultural Factors . . . . . . . . . . . . . . . . . . 83

3.5 Empirical Findings . . . . . . . . . . . . . . . . . . 85

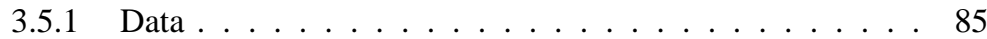

3.5.2 Descriptive Statistics . . . . . . . . . . . . . . . 90

3.5.3 Method .................... . 95

3.5.4 Regression Results . . . . . . . . . . . . . . . . . . . 98

3.6 Conclusion . . . . . . . . . . . . . . . . 111 


\section{List of Tables}

1.1 Poverty in Peru - Difference in Dominance Surfaces (1998/99 1999/2000) . . . . . . . . . . . . . . . . 22

1.2 Poverty in Peru - Difference in Dominance Surfaces (1998/99 2000/01) . . . . . . . . . . . . . . . . . . 24

1.3 Poverty in Peru - Difference in Dominance Surfaces (1998/99 2000/02) . . . . . . . . . . . . . . . 26

1.4 Poverty in Indonesia - Differences in dominance surfaces (Selfemployed - Private sector) _ . . . . . . . . . . . . 27

1.5 Relative Poverty in Indonesia - Difference in Dominance Surfaces (1993/97 - 1997/2000) . . . . . . . . . . . . . . . 29

1.6 Relative Poverty in Peru and Indonesia - Difference in Dominance Surfaces Peru (1997/2000) - Indonesia (1997/2000) . . . . 30

1.7 Poverty in Peru - Difference in Dominance Surfaces for Several Construction Modes of Time Spans . . . . . . . . . . . . . . . . 32

2.1 Descriptive Regional Growth and Poverty Statistics . . . . . . . 37

2.2 Determinants of spatial inequality . . . . . . . . . . . . 42

2.3 Models - 1994 - Fixed effects . . . . . . . . . . . . . . . . . . 50

2.4 Models - 1998 - Fixed effects . . . . . . . . . . . . . . . . 51

2.5 Models - 2003 - Fixed effects . . . . . . . . . . . . . . . 54

2.6 Intraclass correlation coefficients (ICC) . . . . . . . . . . 55

2.7 Proportional change of variance components . . . . . . . . . . . 56

2.8 Contribution of observed and unobserved characteristics on the variation on each level . . . . . . . . . . . . . . . . 60

2.9 Models - 1994 - Random effects . . . . . . . . . . . . . . . . . . 62

2.10 Models - 2003 - Random effects . . . . . . . . . . . . . . . . 62

2.11 Models - 1998 - Random effects . . . . . . . . . . . . . . 63

3.1 Mortality and undernutrition rates in the SSA context . . . . . . 73

3.2 Classification of Variables Influencing Health and Mortality Out-

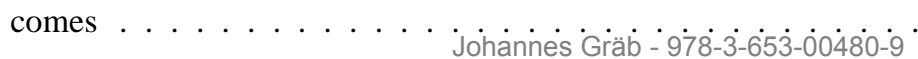


3.3 Description on variables of interest . . . . . . . . . . . . 87

3.4 Descriptive statistics on variables of interest . . . . . . . . . . 91

3.5 Determinants of stunting . . . . . . . . . . . . . 100

3.6 Determinants of wasting . . . . . . . . . . . . 101

3.7 Determinants of under5-mortality . . . . . . . . . . . . . 104

3.8 Variance of family random effect . . . . . . . . . . . . . 110

A.9 Regional Codes . . . . . . . . . . . . . . . . . 113 


\section{List of Figures}

1.1 Test Domain for Dynamic Poverty Comparisons . . . . . . . . . . 15

1.2 Poverty in Peru: Dominance Surface of the Time Span 1998/99 . . 21

1.3 Poverty in Peru: Difference in Dominance Surfaces (1998/99 1999/2000) . . . . . . . . . . . . . . . . 22

1.4 Poverty in Peru: Difference in Dominance Surfaces (1998/99 2000/01) . . . . . . . . . . . . . . . . . 24

1.5 Poverty in Peru: Difference in Dominance Surfaces (1998/99 2000/02) . . . . . . . . . . . . . . . . 26

1.6 Relative poverty in Indonesia: Difference in Dominance Surfaces (1993/97-1997/2000) . . . . . . . . . . . . . 29

2.1 Growth and Poverty Incidence on Provincial Level _ . . . . . . 38

2.2 Convergence in Burkina Faso, initial per capita income and growth on the department level (135 observations), 1994-2003 . . . . . . 39

3.1 Stunting rates in SSA . . . . . . . . . . . . 74

3.2 Wasting rates in SSA . . . . . . . . . . . . . 74

3.3 Theoretical Framework . . . . . . . . . . . . . . . . 76

3.4 Water Requirement Satisfaction Index (WRSI) . . . . . . . . 80

3.5 Normalized Difference Vegetation Index - Kenya . . . . . . . . . 81

3.6 Climate Suitability for Endemic Malaria . . . . . . . . . . . . . 82

A.7 Map of Burkina Faso . . . . . . . . . . . . . . . . . . 113

A.8 BLUP of HH Size - $1994 \ldots \ldots \ldots \ldots$

A.9 BLUP of HH Size $-1998 \ldots \ldots \ldots \ldots \ldots$

A.10 BLUP of HH Size $-2003 \ldots \ldots \ldots \ldots$

A.11 Youth per Adult $-1994 \ldots \ldots$. . . . . . . . . . . 114

A.12 Children per Adult - 1998 . . . . . . . . . . . . . . . . 114

A.13 Youth per Adult -2003 . . . . . . . . . . . . . . . . 114

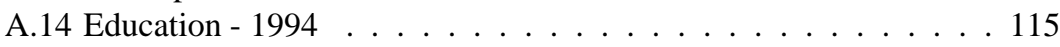

A.15 Education $-1998 \ldots \ldots \ldots \ldots \ldots$

A.16 Education $-2003 \ldots \ldots \ldots \ldots \ldots$ 
Johannes Gräb - 978-3-653-00480-9

Downloaded from PubFactory at 01/11/2019 02:31:01AM

via free access 


\section{List of Abbreviations}

$\begin{array}{ll}\text { AIC } & \text { Akaike Information Criterion } \\ \text { AIDS } & \text { Acquired Immunodeficiency Syndrome } \\ \text { AVG } & \text { Average } \\ \text { BLUP } & \text { Best linear Unbiased Estimator } \\ \text { BMI } & \text { Body Mass Index } \\ \text { Cons } & \text { Constant } \\ \text { CV } & \text { Coefficient of Variation } \\ \text { Cov } & \text { Covariance } \\ \text { CPI } & \text { Consumer Price Index } \\ \text { DHS } & \text { Demographic and Health Survey } \\ \text { DM } & \text { Direction De La Meteorologie Burkina Faso } \\ \text { MI } & \text { Ministere Des Infrastructures } \\ \text { EA } & \text { Enquete Agricole } \\ \text { EC } & \text { Enquete Communitaire } \\ \text { ENAHO } & \text { Peruvian Encuesta Nacional de Hogares } \\ \text { EP } & \text { Enquete Prioritaire } \\ \text { EXP } & \text { Expenditure } \\ \text { Est } & \text { Estimate } \\ \text { FEWS } & \text { Famine Early Warning System } \\ \text { FGT } & \text { Foster Greer Thorbecke } \\ \text { FSD } & \text { First Order Stochastic Dominance } \\ \text { GIS } & \text { Geographic Information System } \\ \text { GPS } & \text { Geographic Positioning System } \\ \text { HH } & \text { Household } \\ \text { HIV } & \text { Human Immunodeficiency Virus } \\ \text { ICC } & \text { Intraclass Correlation Coefficient } \\ \text { IFLS } & \text { Indonesian Family Life Survey } \\ \text { KDHS } & \text { Kenyan Demographic and Health Survey } \\ \text { Lit } & \text { Literate } \\ \text { LR } & \text { Likelihood Ratio } \\ \text { LSMS } & \text { Living Standard Measurement Survey } \\ & \end{array}$


MARA Mapping Malaria risk in Africa

NASA National Aeronautics and Space Administration

No Number

NDVI Normalized Difference Vegetation Index

Obs Observations

OLS Ordinary Least Squares

P0 Poverty Headcount

PC Per Capita

POP Population

PPP Purchasing Power Parity

RC Random Coefficient

SSA Sub-Saharan Africa

Std dev Standard Deviation

SE Standard Error

Tempmax Maximum Temperature

PCA Principal Component Analysis

PWT Penn World Tables

SSD Second Order Stochastic Dominance

U5M Under 5 Mortality

UN United Nations

UNDP United Nations Development Programme

UNICEF United Nations Children's Fund

USD United States Dollar

Var Variance

WHO World Health Organization

WRSI Water Requirement Satisfaction Index

WTO World Trade Organization

ZD Zone Denombrement 


\section{Introduction and Overview}

Following the proclamation of the Millennium Development Goals, the public perception of the miserable living conditions of the developing countries' poor people increased considerably. More than a billion people declared to be poor cautioned society, political authorities and economic researchers to deal more intensively with the extreme harm of the poor. Poverty developed to one of the main topics of present economic policy debates. Recent World Economic Summits of Heiligendamm and Toyako dealt specifically with the economic development of Africa and Asia to reduce global poverty and with the political action necessary to provide food safety all over the world. Privately organized events like 'Live 8 ' or 'Stand Against Poverty' encouraged, globally, thousands of people to take action against poverty by jointly pressuring political leaders to increase financial aid. Public and private efforts aimed at implementing poverty alleviation policies to decrease the amount of people suffering under poor housing conditions, inadequate nutritional intake or insufficient education.

Implementing successful poverty alleviation policies requires essential targeting of poverty causing factors. Therefore policy makers are in need of (1) a concept of poverty and its classification, (2) a thorough understanding of the underlying mechanism that lead to poverty and (3) empirical methods to analyze (1) and (2). This dissertation claims to contribute to the latter by proposing and applying specific statistical methodologies to analyze the extent of poverty and its underlying factors based on recent household surveys in developing countries.

\section{Empirical development economics}

The measurement of poverty and the analysis of poverty-causing factors are largely based on the application of statistical methods. As in economics generally, research on poverty focusses particularly on issues, where empirical data is available, by formulating theoretical hypotheses - most often on causal relationships which will then be tested by estimation. There is perhaps no other area of science, where the application of quantitative methods on statistical data to test theoretical assumptions is as prominent as in economics. The prevalent application of math- 
ematical statistics in empirical economics lead Ragnar Frisch and Joseph Schumpeter as early as the 1930's to constitute the term econometrics and to establish the Econometric society. In the editorial of the first issue of Econometrica Frisch (1933) noted: "Experience has shown that each of those three viewpoints, that of statistics, economic theory, and mathematics, is a necessary, but not by itself a sufficient, condition for a real understanding of the quantitative relations in modern economic life. It is the unification of all three that is powerful. And it is this unification that constitutes econometrics."

While much of early development economics was entirely theoretical (see e.g. Rosenstein-Rodan (1943); Leibenstein (1957); Sen (1973)), there has been a clear shift in the last two decades towards mainstream empirical, i.e. econometrical, economics (Ray, 2007). As Mookherjee (2005) notes: "Development economics is increasingly becoming an empirical discipline today." This phenomenon can be ascribed to two main trends of recent decades. On the one hand, technical progress and the implementation of user friendly statistical software greatly facilitated the application of complex and computationally extensive statistical methods. More importantly, however, data availability expanded extensively. Since the beginning of the 1990s, household survey data, providing requisite information at the individual level, has become available for most developing countries. Application of miscellaneous methodologies has since enabled researchers to measure and compare the extent of poverty, to identify and quantify its driving factors or to evaluate competing poverty reduction strategies.

The present prominence of empirics has recently brought a discussion into development economics that has already had a longstanding tradition in mainstream economics. The discussion is about the actual value added of empirical research. There are basically two main concerns. (i) The econometric validity of empirical results is often, especially in the case of causal regression analysis, disputable. The key problem in regression analysis is to infer causality from simple correlation. Seven decades ago, Keynes (1939) already expressed his concern about the usefulness of causal inference based on regression analysis by commenting on the "slippery problem of passing from statistical description to inductive generalization in the case of simple correlation". (ii) The first concern directly provokes the second. To circumvent possible econometric biases empirical papers focus nowadays on combating the econometric problems. Therefore, authors concentrate on specific phenomenon that may be analyzed, given the data, in a solid way. This comes at a cost: generalization of the often microscopic results is seldom reasonable. ${ }^{1}$

${ }^{1}$ For a recent debate on theory versus empirics in development economics see Mookherjee (2005) whose article is followed by comments from Bardhan (2005), Basu (2005), Banerjee (2005), and Kanbur (2005). For a general critique on the application of econometrics see Hendry (1980). 
These concerns have clearly to be taken into account for further (development) economic research. There is, however, no doubt that much progress has already been made to circumvent some of the cumbersome problems of econometrics and that empirical findings have, in Ragnar Frisch's sense, indeed provided notable contributions for a better understanding of the quantitative relations in modern economic life.

From a development economics perspective, empirical methods have proved to be particularly useful in poverty analysis. Research on poverty measurement facilitated the comprehension of the extent of poverty and its evolution over time and space. In a recent contribution, Ravallion et al. (2008) used regression analysis to revise the international " $\$ 1$ a day" poverty line. Based on new empirical data, the authors propose an absolute international poverty line of $\$ 1.25$. Empirical findings like these contribute to poverty reduction by enabling decision makers to target specifically those groups of the world population who are in particular need of poverty alleviation programmes.

Such as poverty measurement, causal poverty analysis may contribute significantly to poverty reduction by identifying driving factors of poverty. To circumvent the problem of endogeneity described above, recent contributions have focussed on the application of instrumental variables in regression analysis and the use of randomized controlled trials. Randomized controlled trails (RCT) involve the random allocation of different interventions (treatments or conditions) to subjects to create exogenous benchmark groups. If RCT are not available, econometric methods may help to create artificial experiments which may then serve as benchmarks. Using a RCT, Miguel and Kremer (2004) found that children going to schools, where de-worming medicine was distributed, came to school more regularly. ${ }^{2}$ In a similar paper Chattopadhyay and Duflo (2004) conclude that panchayats ${ }^{3}$ headed by a woman are performing significantly better, e.g. in the provisioning of water. Identified driving factors of poverty, like women as political leaders or de-worming of children, serve as starting point to deduce effective policy interventions.

The papers of Ravallion et al. (2008), Miguel and Kremer (2004) and Chattopadhyay and Duflo (2004) show two things: (i) the relevance of empirical development economics for effective poverty reduction policies and (ii) the impressive progress of empirical analysis since the times of Keynes (1939) or Hendry (1980).

The enormous potential of statistical analysis in poverty research has, however, still not been sufficiently exploited. Quite the contrary, the scope for analytical poverty research widens continuously: constant appearance of new compre-

\footnotetext{
${ }^{2}$ In this case, the data was based on a RCT since de-worming medicine was distributed to children of randomly assigned schools in Western Kenya.

${ }^{3}$ Panchayats are local government bodies at the village level in India. 
hensive data sets, such as the release of household panel data in more and more developing countries, broadens the area of application while rapid progress in natural science entails steady development of new methodologies.

This dissertation is a collection of three independent essays that deal with the application of econometric methods, based on household survey data, in the context of poverty research. All studies consider well-known statistical concepts that have yet not been applied to the research questions under consideration. By this means, the papers contribute to research in development economics in two ways. The application of recent methodologies allows gaining better insight and deriving new findings on the measurement of poverty and its underlying factors. Simultaneously, the application is conducive to narrow the gap between the comprehensive statistical toolbox and its still limited application in development economic research.

The first chapter deals with the concept of poverty comparisons when the wellbeing indicator, income, is observed over consecutive periods. The second chapter studies the determinants of spatial inequality in household income in Burkina Faso, by decomposing overall inequality in inequality within and between nested spatial levels. The third chapter analyzes the relation between a child's nutritional status, derived from its stunting and wasting z-score, and its survival probability, to resolve the paradox of high mortality but low malnutrition rates in the Lake Victoria region of Kenya.

\section{Three essays in empirical development economics}

\section{A concept for multiperiod poverty comparisons}

Research on poverty measurement is closely connected to the seminal work of Sen (1976). Sen distinguishes between two fundamental issues: identifying the poor within the population by setting a poverty line and constructing a poverty index to measure the extent of deprivation. Based on an axiomatic approach Sen constructs a poverty measure capable of performing ordinal welfare comparisons.

Following Sen, research on poverty measurement evolved into two strands of literature: (i) the construction of poverty indices to measure poverty and (ii) the generation of poverty orderings to compare poverty. The first strand, poverty measurement, deals with the attempt to construct summary poverty indices that capture several concepts of poverty and satisfy various poverty axioms (see e.g. Foster et al. (1984); Atkinson (1987); Zheng (1997)). Beyond the poverty head count, to measure the fraction of people below the poverty line, the concepts include, among others, the poverty gap to capture the average extent of individual poverty and the squared poverty gap to measure the inequality among the poor. Among the numerous proposed poverty axioms, researchers agreed on a core set 
of axioms each poverty measure should satisfy: focussed, continuous, monotonic and distribution sensitive. ${ }^{4}$

The various proposed measures impose implicitly the need for the second strand of the literature. Any choice of poverty measure is arbitrary and may lead to different outcomes. The second strand, poverty orderings, approaches exactly this arbitrariness by proposing methodologies that yield rankings of poverty which are robust to alternative poverty measures. Another arbitrariness in poverty measurement results from the setting of the poverty line. Poverty line construction is usually based on minimum nutritional intake. Since there is no exact level of food intake requirements different reasonable poverty lines are supposable (Atkinson, 1983). The literature on poverty orderings examines the rankings of distributions of one or more indicators of well-being to yield poverty comparisons which are robust to a wide range of poverty measures and poverty lines (see e.g. Atkinson and Bourguignon (1982); Atkinson (1987); Foster and Shorrocks (1988a,b,c); Duclos et al. (2006b)).

Essay 1 follows the second strand of literature by elaborating a concept for multiperiod poverty comparisons. Above the choice of a suitable poverty measure and poverty line, the paper deals with the question how poverty can be measured and compared when the indicator of well being is observed over several points in time. Specifically, Essay 1 proposes a concept to compare an individual's well-being over consecutive periods as well as to compare well-being of two different individuals observed at two concurrent periods. The proposed methodology allows for multiperiod poverty comparisons that are robust to any specific poverty index, to any arbitrary setting of the poverty line and to any aggregation procedures of individual incomes over time. The elaborated concept, which is, following Atkinson (1987) and Duclos et al. (2006b), based upon the stochastic dominance methodology, is illustrated by performing multiperiod poverty comparisons for Indonesia and Peru. Showing considerable dependence of poverty orderings on the aggregation procedures of income over time, the results emphasize the relevance of the approach.

\section{Econometric analysis of spatial inequality}

Causal poverty analysis, based on regression models, allows identifying driving factors of households' living standards. A peculiar discussion has taken place in development economics about the importance of geographic factors in explaining observed spatial variation in household income (see e.g. Ravallion and Wodon (1999); Jalan and Ravallion (2002); De Vreyer et al. (2009); Grimm and Klasen

\footnotetext{
${ }^{4}$ For a discussion of relevant axioms see Zheng (2000). 
(2008)). ${ }^{5}$ Several developing economies show areas that are persistently poor. Two divergent views exist in explaining why some regions perform better than other regions within the same country. Areas could be poor due to a spatial concentration of households with similar, poor, characteristics. According to this view, geographic endowments do not play a role in determining households' income. On the other hand, geographic capital might be correlated with living standards of different regions. Differences in area-specific factors, like climate or altitude in terms of pure geographic factors, or infrastructure in terms of area endowments, may directly have a causal role in determining households' welfare.

Using different regression techniques Ravallion and Wodon (1999), Jalan and Ravallion (2002) and Benson et al. (2005) analyze if differences in households' living standards across spatial entities within a country are entirely accountable to a spatial segregation of people with similar endowments, or to geography per se. All studies conclude that it is not solely a spatial correlation of differences in mobile non geographic characteristics that makes areas poor. Specific factors of a households area of residence matter by restraining households income growth and by altering returns to private endowments.

While all these studies suggest that poverty reduction efforts have to be targeted at the sub-national level, they do not provide a decomposition of the variance in living standards observed within and between nested spatial units. Consequently, the studies cannot weight the influence of the different spatial units on the variance in income levels. Essay 2 suggests a novel methodology to address this issue by building a multilevel random coefficient model able to decompose the variance in living standards across four spatial levels; households, communities, provinces and (agro-climatic) regions. Knowledge of the relevance of each spatial level for household income generation is particularly important from a political point of view: Since there may be constraints on the ability to target household characteristics, targeting spatial units effectively seems crucial.

Based on the proposed multilevel modeling approach, Essay 2 decomposes the sources of spatial disparities in incomes among households in Burkina Faso. The results show that spatial disparities are not only driven by the spatial concentration of households with particular endowments but to a large extent also by disparities in community endowments. Climatic differences across regions do also matter, but to a much smaller extent.

\footnotetext{
${ }^{5}$ For a discussion on the importance of geographic factors in a cross country setting see e.g. Acemoglu et al. (2001); Hall and Jones (1999); Gallup et al. (1998). 


\section{Empirical analysis of child mortality and undernutrition}

Essay 1 and Essay 2 focus on monetary poverty, i.e. low levels of income and consumption, respectively. Households in developing countries also suffer from other dimensions of poverty. While poverty has initially been regarded as a monetary phenomenon, its multidimensionality is now widely accepted (Sen, 1987; Strauss and Thomas, 1998). Most of the concepts for the measurement and analysis of poverty have therefore been developed in a way that they are also applicable to non-monetary indicators of well-being, such as the health, nutritional or educational status of an individual (see e.g. Bourguignon and Chakravarty (2003); Duclos et al. (2006b). Deprivation in the multiple dimensions of poverty should hence be taken into account when measuring poverty, and the underling mechanism that lead to observed outcomes should be analyzed to deduce effective poverty reduction policies.

Two of the still most challenging problems in the fight against poverty are the prevalent high rates of undernutrition and child mortality. One of the major causes of child mortality is thought to be undernutrition itself. Pelletier et al. (1995) claim that undernutrition is the underlying cause of more than $50 \%$ of all child deaths in the world. The close relationship between a child's nutritional status and its survival probability is challenged when nutrition and mortality outcomes are analyzed in the Lake Victoria Region of Kenya. Essay 3 shows that there is no other region in Sub-Saharan Africa where the pattern of low levels of malnutrition together with dramatically high rates of mortality is as pronounced as around Lake Victoria.

Essay 3 investigates the role of cultural, geographic, and political factors on the relationship of anthropometric outcomes of children and Under-5 mortality rates in Kenya with an explicit focus on the unique situation of the territory around Lake Victoria. Based on linear and nonlinear multilevel regression analysis to control for unobserved household and community characteristics the driving factors of mortality, stunting, and wasting are analyzed jointly.

The findings point to a unique interplay of cultural, geographic and political factors in the Lake Victoria region which are responsible for causing the described paradox. The results do not only demonstrate the relevance of considering and understanding the country specific context when analyzing child health outcomes but also that the common practice to make inferences about health status based on anthropometric outcomes has to be done with strong caution and can easily lead to erroneous results. 
Johannes Gräb - 978-3-653-00480-9

Downloaded from PubFactory at 01/11/2019 02:31:01AM

via free access 


\title{
Essay 1
}

\section{Robust Multiperiod Poverty Comparisons}

\begin{abstract}
We propose a methodology for comparing poverty over multiple periods across time and space that does not arbitrarily aggregate income over various years or rely on arbitrarily specified poverty lines or poverty indices. We use multivariate stochastic dominance tests to create dominance surfaces for different time spans. We elaborate the method first for the bidimensional case, using as dimensions income observed over two periods: one at the beginning and one at the end of a time span. Subsequently, we extend it to the case where incomes are observed over $n$-periods. We illustrate our approach by performing poverty comparisons using data for Indonesia and Peru.
\end{abstract}

based on joint work with Michael Grimm. 


\subsection{Introduction}

Today it is well established that poverty is a dynamic phenomenon. But if poverty does fluctuate and evolve over time, this raises the question of how best to measure it over multiple periods. Cross-sectional poverty measures can provide abundant information on the extent of poverty at a given point, but almost none on the rate at which people escape from or fall into poverty over time.

Recognizing this, authors such as Grootaert and Kanbur (1995) have suggested focusing on households' changes in poverty status. Others have developed concepts to aggregate incomes over multiple periods (i.e., trajectories of income over time) using an evaluation function that explicitly captures, for example, the risk aversion of households (see e.g., Cruces (2005)). While such an approach has the advantage of accounting for the negative effects of income variability on the wellbeing of households, it requires arbitrary assumptions about how exactly 'riskadjusted mean income' is best computed.

Likewise, considering the standard spells and component approaches proposed for measuring and conceptualizing chronic and transient poverty, one can safely state that the results and consequently the policy implications depend heavily on how the two forms of poverty are measured: how incomes are aggregated over time, how the poverty line is set, and what poverty index is chosen (see, e.g., Hulme and McKay (2005); Jalan and Ravallion (1998); Duclos et al. (2006a)). Both the spells and component approach usually rely on one specific poverty line and one specific poverty function. Moreover, approaches based on the components approach are usually based on some calculation of average income over time and thus abstract from the exact pattern of the income trajectory. In other words, three consecutive years of high income followed by three consecutive years of low income are treated as six years over which a year of high income follows a year of low income and so on.

To circumvent these problems, we suggest another approach for multiperiod poverty measurement based on stochastic dominance tests. This enables us to establish poverty orderings that are valid for a wide range of aggregation rules of incomes observed over time, a wide range of poverty indices, and a wide range of poverty lines. Our approach relies on the literature on multi-dimensional poverty orderings Duclos et al. (2006b), in which dimensions refer to various indicators of individual well-being such as income, education and health. ${ }^{1}$ Our dimensions are incomes observed at different points in time. Defining dimensions in this way raises some further challenges, which we discuss below. We develop our approach first for the case where incomes are observed over two periods and then extend it

\footnotetext{
${ }^{1}$ See also Duclos et al. (2006c) and the seminal papers by Bourguignon and Chakravarty (2002, 2003) 
to the case where incomes are observed over $n$-periods. We illustrate this approach using longitudinal data for Indonesia and Peru. Note that we do not address the issue of income uncertainty and disutility due to income volatility.

Among the papers dealing with multiperiod poverty, probably Hoy and Zheng (2007), Foster (2007) and Bossert et al. (2008) are the closest related to ours.

Hoy and Zheng (2007) suggested a lifetime poverty measure derived from an axiomatic approach. If we computed the poverty measure we suggest for a lifetime period instead for sub-periods of total lifetime, we would be able to derive similar results. However, we do not explore the implications of various axioms one may wish to impose on such a lifetime poverty measure. Moreover, whereas Hoy and Zheng's approach is designed to compare lifetime poverty across different groups of individuals, our approach is intended to do both, either compare multiperiod poverty across different sub-periods of total lifetime for a given group of individuals or to compare multiperiod poverty for a given sub-period across different groups of individuals.

In the spirit of Hoy and Zheng (2007), Bossert et al. (2008) have also constructed a lifetime poverty measure. Their approach differs in the properties that are deemed relevant. Bossert et al. (2008) model explicitly the persistence of poverty over time by relaxing the notion of path independence considered by Hoy and Zheng (2007). Their index regards the negative effects of being in poverty as cumulative, in the sense that a two-period poverty spell is worse than two oneperiod spells interrupted by a period out of poverty.

Foster (2007) suggested a new family of chronic poverty measures based on the well-known Foster-Greer-Thorbecke measures (1984). Foster (2007) identifies the chronically poor using two cutoffs: a standard poverty line, which identifies the time periods during which a person is poor, and a duration cutoff, which is the minimum percentage of time a person must be in poverty in order to be chronically poor.

The remainder of our paper is organized as follows. In Section 2 we present our methodology. In Section 3 we implement our methodology empirically and analyze multiperiod poverty in Indonesia and Peru. In Section 4 we discuss our results and conclude.

\subsection{Methodology}

\subsubsection{Stochastic dominance in a one-period welfare measure}

We assume that individual well-being, $\lambda$, is a function of $y$, a well-being indicator, for instance income received in period $t$. Let $y$ be defined over the interval $[0, \infty]$, where the set of distributions of well-being indicators is $\Psi:=F:[0, \infty] \rightarrow[0,1]$. 
We assume a non-decreasing well-being function without imposing anything concerning the exact contribution of $y$ to well-being:

$$
\lambda(y), \text { where } \frac{\partial \lambda(y)}{\partial(y)} \geq 0 .
$$

An individual is assumed to be poor if well-being $\lambda(y)$ is below a poverty frontier, $\lambda(z)$, where $z$ is the poverty line belonging to the well-being indicator. The poverty set can then be defined as:

$$
\Lambda(\lambda)=\{y \mid \lambda(y) \leq \lambda(z)\},
$$

with $\lambda(z)=0$.

In what follows we consider, following Atkinson (1987), all additively separable poverty measures $P$ that are non decreasing in $\lambda(y)$ and anonymous. We denote this set of poverty measures $\Xi_{1}$. Our poverty measure can be computed by:

$$
P(F ; \lambda)=\int_{\Lambda(\lambda(z))} p(\lambda(y), \lambda(z)) d F(y)
$$

If well-being is only measured along one dimension, e.g. the one period case, equation 1.3 can be rewritten as:

$$
P(F ; z)=\int_{0}^{z} p(y, z) d F(y) .
$$

Our set of poverty measures, $\Xi_{1}$, includes, for instance, the Watts measure of poverty (Watts, 1968), where $p(y, z)=(\ln z-\ln y)$, and all poverty measures within the Foster-Greer-Thorbecke family, $P_{\alpha}$ (Foster et al., 1984) with $\alpha \geq 0$ (Foster and Shorrocks, 1988b,c), where $p(y, z)=(1-y / z)^{\alpha}{ }^{2}$

Tests of stochastic dominance are today widely used to establish poverty orderings $\mathbf{D}$ that are robust for a broad class of poverty measures, $P(F ; z)$, and a large range of poverty lines, $z \in[0, \infty]$.

Given two distributions $F \in \Psi$ and $G \in \Psi$, the first order stochastic dominance condition (FSD), $\mathbf{D}_{\mathbf{1}}$, states:

$$
F \mathbf{D}_{\mathbf{1}} G \forall P \in \Xi_{1}, z \in\left[0, z^{\max }\right] \Longleftrightarrow F(z)-G(z)<0 \forall z \in\left[0, z^{\max }\right],
$$

where $F \mathbf{D}_{1} G$ means that $F$ has unambiguously less poverty than $G$ with respect to all poverty indices belonging to the class $\Xi_{1}$ and all poverty lines within the range $\left[0, z^{\max }\right]$.

\footnotetext{
${ }^{2}$ The Foster-Greer-Thorbecke poverty measure has the formula $P_{\alpha}=1 / N \sum_{i=1}^{n}\left(1-\frac{y_{i}}{z}\right)^{\alpha}$, where $N$ is the total number of individuals $i=1, \ldots, N$. The parameter $\alpha>0$ is a poverty aversion parameter: $\alpha=0$ yields the poverty headcount index, $\alpha=1$ the poverty gap index, and $\alpha=2$ poverty severity index (Foster et al., 1984).
} 
For FSD orderings it is sufficient to compare the distribution function of the well-being indicator in period $1, F\left(y_{1}\right)$, with its analog in period 2, $G\left(y_{2}\right)$. The distribution function can also be called dominance curve. If first order stochastic dominance does not hold, higher-order stochastic dominance tests can be applied to generate robust poverty orderings. Higher-order dominance requires to add further assumptions on how the function $p(y, z)$ evolves with $y$. For instance, second order stochastic dominance (SSD) requires to specify $p(y, z)$ in a way that $P$ satisfies the Pigou-Dalton transfer principle (see e.g. Foster and Jin (1996)). The Pigou-Dalton transfer principle states that a transfer of income from a richer to a poorer person will not increase poverty as long as that transfer does not reverse the ranking of the two. In this case, the areas under the distribution functions can be compared to generate poverty orderings. If we denote the set of all Daltonian poverty measures, $\Xi_{2}$, then $\operatorname{SSD}, \mathbf{D}_{\mathbf{2}}$, states:

$$
F \mathbf{D}_{2} G \forall P \in \Xi_{2}, z \in\left[0, z^{\max }\right] \Longleftrightarrow \int_{0}^{z} F(y) d y-\int_{0}^{z} G(y) d y<0 \forall z \in\left[0, z^{\max }\right]
$$

where $F \mathbf{D}_{2} G$ means that $P(F)$ has unambiguously less poverty than $P(G)$ with respect to all poverty indices belonging to the class $\Xi_{2}$ and all poverty lines within the range $\left[0, z^{\max }\right]$. For instance, within the FGT poverty measure family the poverty gap $(\alpha=1)$ satisfies the Pigou-Dalton transfer principle, the poverty headcount $(\alpha=0)$ does not.

If second order dominance does also not hold, it is possible to integrate the distribution function again and to test for third order dominance. This would of course further limit the set of applicable poverty measures by imposing even more restrictive axioms. Therefore, in the theoretical part of our paper, we restrict our analysis to FSD and SSD. In the empirical part we consider only FSD.

Note also that we do not consider weak stochastic dominance, because statistically it is impossible to distinguish weak and strong stochastic dominance. ${ }^{3}$

It is widely acknowledged that the concept of poverty dominance is useful because it circumvents the problem of choosing one particular poverty measure and one specific poverty line. In the following, we extend the concept, first to two-period welfare measures and then to $n$-period welfare measures.

\subsubsection{Stochastic dominance in a two-period welfare measure}

To take into account the dynamic aspects of poverty, we now extend the one-period well-being function to a two-period well-being function, where the arguments are

\footnotetext{
${ }^{3}$ Weak stochastic dominance requires $F(z)-G(z) \leq 0$ for all poverty measures. Thus strict stochastic dominance, as defined in equation 1.5 , implies weak stochastic dominance. 
$\left(y_{1}, y_{2}\right)$, e.g. income received in periods 1 and 2 . The well-being function can then be written as:

$$
\lambda\left(y_{1}, y_{2}\right): \Re^{2} \rightarrow \Re \mid \frac{\partial \lambda\left(y_{1}, y_{2}\right)}{\partial\left(y_{1}\right)} \geq 0, \frac{\partial \lambda\left(y_{1}, y_{2}\right)}{\partial\left(y_{2}\right)} \geq 0 .
$$

Hence, we impose the condition that the well-being function, $\lambda$, is differentiable with respect to the welfare measure in $t=1$ and $t=2$ and that income in both periods contributes positively to individual well-being. Yet, as before, we impose nothing regarding the precise value of the contribution of each year to individual well-being.

We define an individual to be poor if his or her overall well-being $\lambda\left(y_{1}, y_{2}\right)$ is below the unknown poverty frontier. In the two-period case, the poverty frontier is not a single point, $z$, but a locus of points. We define this locus as $\lambda\left(y_{1}, y_{2}\right)=0$. The overall set of poor people is defined as:

$$
\Lambda(\lambda)=\left\{y_{1}, y_{2} \mid \lambda\left(y_{1}, y_{2}\right) \leq 0\right\} .
$$

Depending on the specific definition of the locus of the poverty frontier, multiperiod poverty comparisons can be performed according to the 'intersection' and the 'union' poverty definition (Duclos et al., 2006b). Intersection poverty means in our case that someone is considered poor if well-being is below the poverty threshold in both periods. The concept of 'intersection' multiperiod poverty is therefore closely related to the concept of chronic poverty (see e.g. Hulme and Shepherd (2003)). Intersection poverty is represented in figure 1.1 by the crossbred-shaded area under the function $\lambda_{1}\left(y_{1}, y_{2}\right)$ (dashed line). Union poverty means that someone is considered poor if well-being is below the poverty threshold in one of the two periods. This is represented in figure 1.1 by the entire shaded area under the function $\lambda_{2}\left(y_{1}, y_{2}\right)$ (dotted line). In the empirical part of our paper we emphasize the parallels with the concept of chronic poverty and thus focus on intersection poverty.

As in the one-period case, we consider all additively separable, non decreasing and anonymous poverty measures $P$. However, we add a further restriction. We require $y_{1}$ and $y_{2}$ to be substitutes in $\lambda\left(y_{1}, y_{2}\right) .{ }^{4}$ This assumption implies that an increase of the well-being indicator in one period increases well-being more the lower the well-being indicator in the other period. Hence, our concept of multiperiod poverty accounts for the correlation between individuals' outcomes across both periods. We denote this set of poverty measures $\Xi_{1,1}$. Transferring equation 1.4 to the two-period case, the poverty measure reads:

$$
P(F ; \lambda)=\iint_{\Lambda\left(\lambda\left(z_{1}, z_{2}\right)\right)} p\left(\lambda\left(y_{1}, y_{2}\right), \lambda\left(z_{1}, z_{2}\right)\right) d F\left(y_{1}, y_{2}\right) .
$$

${ }^{4}$ Specifically, we impose: $\frac{\partial \lambda^{2}\left(y_{1}, y_{2}\right)}{\partial y_{1} \partial y_{2}} \geq 0, \forall y_{1}, y_{2}$. 
Figure 1.1: Test Domain for Dynamic Poverty Comparisons

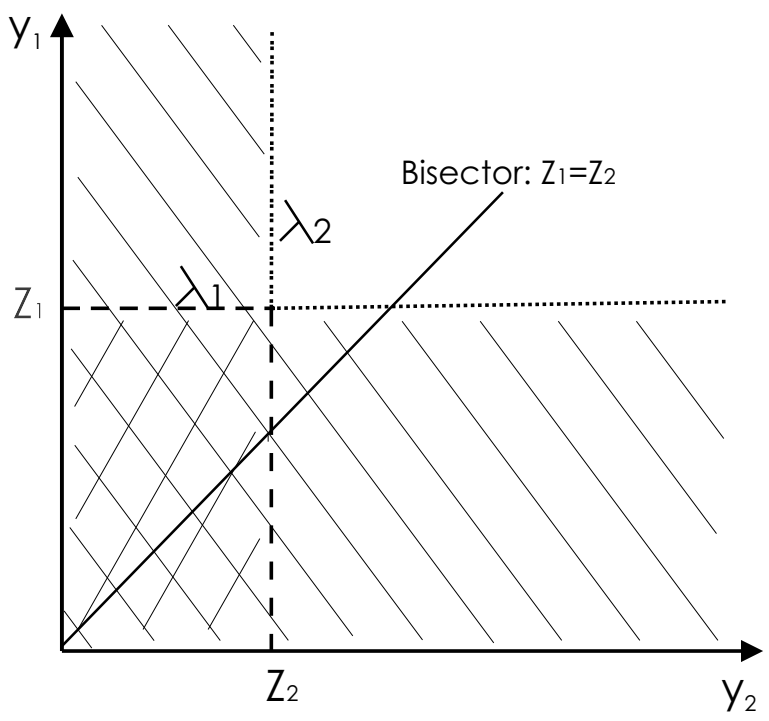

Equation 1.9 holds for multiperiod poverty comparisons according to the intersection as well as according to the union definition of poverty, depending on the locus of $\Lambda(\lambda)$. If we focus on intersection poverty, as we will do in the empirical part, equation 1.9 could be rewritten as:

$$
P\left(F ; z_{1}, z_{2}\right)=\int_{0}^{z_{1}} \int_{0}^{z_{2}} p\left(y_{1}, y_{2}, z_{1}, z_{2}\right) d F\left(y_{1}, y_{2}\right) .
$$

Obviously, as for usual period-by-period poverty orderings, it is desirable that poverty orderings over multiple time spans, $T_{j}$, are robust to a large set of poverty lines $z \in Z$. This can be ensured by simply transferring the concept of stochastic dominance for univariate welfare distributions to the case of bivariate welfare distributions. A comparison of two time spans is denoted in what follows as $T_{a}=\left[t_{1 a} ; t_{2 a}\right]$ vs. $T_{b}=\left[t_{1 b} ; t_{2 b}\right]$, where $t$ now has an index for the period within each time span, year 1 or year 2 , and an index for the time span, time span $a$ or time span $b$.

Furthermore, poverty orderings in the bivariate case, i.e. across time spans, should be robust to a broad range of procedures to aggregate the observed periodspecific well-being indicators over the two periods constituting a time span. Thus, the weight given to each single period should not matter, i.e. whether we weigh each period equally or whether we give to one period a higher weight than to the 
other. A reason for doing the latter might be to account for time preference, i.e. one weighs income today more than income tomorrow. Hence, we require that our ordering is robust to the magnitude and even the sign of the time discount rate. ${ }^{5}$

Given two distributions $F\left(y_{1 a}, y_{2 a}\right) \in \Psi$ and $G\left(y_{1 b}, y_{2 b}\right) \in \Psi$ the first order stochastic dominance condition, $\mathbf{D}_{\mathbf{1 , 1}}$, states:

$$
\begin{aligned}
& F \mathbf{D}_{1,1} G \forall P \in \Xi_{1,1}, z_{1} \in\left[0, z_{1}^{\max }\right], z_{2} \in\left[0, z_{2}^{\max }\right] \\
\Longleftrightarrow & F(z)-G(z)<0 \forall z_{1} \in\left[0, z_{1}^{\max }\right], z_{2} \in\left[0, z_{2}^{\max }\right],
\end{aligned}
$$

where $F \mathbf{D}_{1,1} G$ means that multiperiod poverty is lower over time span $T_{a}$ than over time span $T_{b}$ with respect to all poverty indices belonging to the class $\Xi_{1,1}$ and all poverty within the range $\left[0, z_{1}^{\max }\right]$ and $\left[0, z_{2}^{\max }\right]$.

As in the one-period case, tests of higher order dominance could be equally well established by imposing further assumptions regarding the effect of $y$ on $p\left(y_{1}, y_{2}, z_{1}, z_{2}\right)$. For instance, holding constant the distribution in period 2, we could impose that a transfer from a richer to a poorer person in period 1 reduces poverty. Symmetrically, we would then impose the same transfer sensitivity on period 2.

As mentioned above, we also require our concept to be robust to a broad range of procedures for aggregating the observed period-specific well-being indicators over the two periods constituting a time span. The simplest way to deviate from an aggregation where each period receives the same weight is to vary the poverty lines within time spans, since this varies the income necessary to be beyond the period-specific poverty frontier in each period. If we chose $z_{1} \neq z_{2}$ s.t. $z_{1 a}=z_{1 b}$ and $z_{2 a}=z_{2 b}$, i.e., to give a different weight to the first and second period each time span, the test domain for intersection poverty dominance represents a rectangle, where $y_{1}<z_{1}$ and $y_{2}<z_{2}$. This is illustrated the dashed line in Figure 1.1. In what follows, the aggregation procedure is incorporated through the definition of the poverty lines.

In our methodology, and in contrast to 'one-period-stochastic-dominance', $F\left(y_{1}, y_{2}\right)$ refers now to a bivariate distribution. Hence, the test of stochastic dominance does not imply comparing two curves, as with one-period well-being measures, but two surfaces, where each surface is characterized by its two periods the well-being measure in the first and second period - and the cumulative density at each point of that surface. Rewriting equation 1.10 shows that the dominance

\footnotetext{
${ }^{5}$ In a similar way one could account for uncertainty regarding the right way to deflate incomes from one period to the next.
} 
surface is the product of the two unidimensional curves plus the covariance in the poverty indices in the two dimensions (Duclos et al., 2006b):

$P\left(F ; z_{1}, z_{2}\right)=\int_{0}^{z_{1}} p\left(y_{1}, z_{1}\right) d F\left(y_{1}\right) \int_{0}^{z_{2}} p\left(y_{2}, z_{2}\right) d F\left(y_{2}\right)+\operatorname{cov}\left[\left(p\left(y_{1}, z_{1}\right)\right),\left(p\left(y_{2}, z_{2}\right)\right)\right]$.

The higher the correlation of individuals' incomes the 'higher' the dominance surface. Our multiperiod poverty index therefore implicitly judges a situation in which one individual is always poor and one always rich worse, ceteris paribus, than a situation where two individuals are poor in one period and rich in the other period. A further comment regarding the robustness to the aggregation procedure is in order. In fact, they way we deal with this problem implies that there is one special situation in which robustness to the aggregation procedure cannot be tested. This arises when the time spans under consideration overlap, i.e., when the second period of the first time span simultaneously represents the first period of the second time span. For instance, if poverty over the time span 1980-1990 has to be compared with poverty over the time span 1990-2000, i.e. $y_{2 a}=y_{1 b}$. In this case, the same weight has to be assigned to each.

In this special case the dominance criteria simplifies to:

$$
F \mathbf{D}_{\mathbf{1}, \mathbf{1}} G \forall P \in \Xi_{1,1}, z \in\left[0, z^{\max }\right] \Longleftrightarrow F(z, z)-G(z, z)<0 \forall z \in\left[0, z^{\max }\right]
$$

where $F \mathbf{D}_{\mathbf{1}, 1} G$ means that multiperiod poverty is lower time span $T_{a}$ than over time span $T_{b}$ with respect to all poverty indices belonging to the class $\Xi_{1,1}$ and all poverty lines within the range $\left[0, z^{\max }\right]$. Note that we now only test dominance between the two surfaces along an expansion path of $z$, where $y_{1}<z$ and $y_{2}<z$ (see the bisector line in figure 1.1).

The 'overlap' problem can obviously not occur with comparisons over space, say, if poverty over the timespan 1990-2000 in country a is compared to poverty over the timespan 1990-2000 in country b. In this case nothing prevents us to choose $z_{1} \neq z_{2}$ s.t. $z_{1 a}=z_{1 b}$ and $z_{2 a}=z_{2 b}$, i.e. to give a different weight to the first and second period within each time span in each country.

\subsubsection{Stochastic dominance in a $n$-period welfare measure}

Extending our methodology to the $n$-period case is straightforward. Our wellbeing measure becomes $\lambda\left(y_{1}, y_{2}, \ldots, y_{n}\right)$. The well-being measure is differentiable with respect to each single period income $y_{i}$, where $\partial \lambda\left(y_{1}, y_{2}, \ldots, y_{n}\right) / \partial y_{i} \geq 0$. The poverty locus becomes a $n$-dimensional space.

Given two distributions $F\left(y_{1 a}, y_{2 a}, \ldots, y_{n a}\right) \in \Psi$ and $G\left(y_{1 b}, y_{2 b}, \ldots, y_{n b}\right) \in \Psi$ the first order stochastic dominance condition, $\mathbf{D}_{\mathbf{1}, \mathbf{1}, \ldots, \mathbf{1}}$, states:

$$
F \mathbf{D}_{1,1, \ldots, 1} G \forall P \in \Xi_{1,1, \ldots, 1}, z_{1} \in\left[0, z_{1}^{\max }\right], z_{2} \in\left[0, z_{\text {Jax }}^{\max }\right], \ldots, z_{n} \in\left[0, z^{\max }\right]
$$




$$
\begin{gathered}
\Longleftrightarrow F\left(z_{1}, z_{2}, \ldots, z_{n}\right)-G\left(z_{1}, z_{2}, \ldots, z_{n}\right)<0 \\
\forall z_{1} \in\left[0, z_{1}^{\text {max }}\right], z_{2} \in\left[0, z_{2}^{\max }\right], \ldots, z_{n} \in\left[0, z_{n}^{\max }\right]
\end{gathered}
$$

where $F \mathbf{D}_{1,1, \ldots, 1} G$ means that multiperiod poverty is lower over time span $T_{a}$ than over time span $T_{b}$ with respect to all poverty indices belonging to the class $\Xi_{1,1, \ldots, 1}$ and all poverty lines within the range $z_{1} \in\left[0, z_{1}^{\max }\right], z_{2} \in\left[0, z_{2}^{\max }\right], \ldots, z_{n} \in\left[0, z_{n}^{\max }\right]$.

Of course the $n$-dimensional case allows us again to be robust with respect to the aggregation procedure by giving a different weight to the $n$ periods within each time span, i.e. by choosing $z_{1} \neq z_{2}, \ldots, z_{n-1} \neq z_{n}$ s.t. $z_{1 a}=z_{1 b}, z_{2 a}=z_{2 b}, \ldots, z_{n a}=$ $z_{n b} . F\left(y_{1}, y_{2}, \ldots, y_{n}\right)$ now refers to a $n$-variate distribution and, hence, the test of stochastic dominance now implies comparing two hypersurfaces, where each hypersurface is characterized by its $n$ dimensions - the welfare measure observed over the $n$ periods - and the cumulative density at each point of that hypersurface.

An additional issue that arises in the $n$ period case is how exactly the two time spans are compared. Theoretically, one can compare time spans built using different sets of periods as long as each time span has the same number of periods and as long as the beginning and the end of the first time span each precede the beginning and the end of the second time span respectively. One can then even test for dominance over all these comparisons. Below we illustrate such a case using time spans of a maximum length of four years.

\subsubsection{Relative poverty comparison}

So far we have proposed the methodology of multiperiod poverty comparison for the concept of absolute poverty. Absolute poverty measures deal with income mobility; they consider an absolute poverty frontier and keep track of people who either stay below or cross this fixed frontier. However, the methodology of multiperiod poverty comparisons is equally well applicable to the concept of relative poverty. Relative poverty measures take into account social mobility; while still keeping track of people who either stay below or cross the poverty line, this frontier becomes endogenous, for example, expressed as a ratio of the median income. Embedding our concept of multiperiod poverty in the concept of relative poverty has some common features with the concept of 'social exclusion' as formulated by Bossert et al. (2007).

\subsubsection{Estimation and inference}

To establish first order stochastic dominance empirically, it is sufficient - as shown by Duclos et al. (2006b) - to calculate the differences of $\hat{F}\left(y_{1 a}, y_{2 a}, \ldots, y_{n a}\right)$ and $\hat{G}\left(y_{1 b}, y_{2 b}, \ldots, y_{n b}\right)$ on a sufficiently narrow grid of test points and to test the statistical significance of these differences based on student $t$-tests (where ${ }^{\wedge}$ refers to Johannes Gräb - 978-3-653-00480-9 
estimated values). The relevant test domain changes based on the definition union or intersection - of poverty.

\subsubsection{Bounds to multidimensional dominance}

When applying the methodology presented above, one needs to define a maximum poverty set $\lambda^{*}\left(z_{1}, z_{2}, \ldots, z_{n} \in Z\right)$. Obviously, defining that frontier is always arbitrary. We again follow Duclos et al. (2006b) and estimate that frontier directly from our sample as the maximum $\lambda^{+}$for which multiperiod poverty dominance holds. Then we can locate within $\lambda^{+}$all possible poverty frontiers for which there is necessarily more poverty in time span $a$ than in time span $b$. We then can judge on a case-by-case basis whether these critical sets and frontiers are wide enough to justify the conclusion on poverty dominance.

\subsection{Empirical illustration}

\subsubsection{Data}

To illustrate the methodology presented above, we use longitudinal data for Indonesia and Peru.

For Indonesia, we use all three existing waves of the Indonesian Family Life Survey conducted by RAND, the University of California Los Angeles, the University of Indonesia's Demographic Institute and the Center for Population and Policy Studies of the University of Gadjah Mada in 1993 (IFLS1), 1997 (IFLS2) and 2000 (IFLS3). The IFLS is representative of $83 \%$ of the Indonesian population living in 13 of the (at that time) nation's 26 provinces. The IFLS is judged as having a very high quality, among other things, because individuals who have moved are tracked to their new location and, where possible, interviewed there (for details see Strauss et al. (2004)). Using the three waves, we built two panels one from 1993 to 1997 and one from 1997 to 2000, each comprising roughly 32,000 individuals living in 7,000 households. We use real household expenditure per capita as the welfare measure, but refer to it as income in the following. Expenditure is expressed in 1993 prices and adjusted by regional price deflators to the Jakarta price level.

For Peru we use six waves (1997-2002) of the yearly Peruvian Encuesta Nacional de Hogares conducted by the Instituto Nacional de Estadística e Informática. The ENAHO is representative for the three rural and four urban areas of Peru. The 'panel-households' are only a sub-sample of all households interviewed. Each year, some households drop out of the panel and others are added (rotating panel). We construct several year-to-year panels, each containing, with 
a few exceptions, more than 5,000 individuals living in more than 1,000 households. We use again real household expenditure per capita as the income measure. Expenditure is expressed in 2002 prices and adjusted by regional price deflators to the Lima price level.

To make income comparable between Indonesia and Peru we convert local currencies to international USD. Purchasing Power Parities (PPP) were taken from the Penn World Table 6.1 (see Heston et al. (2002)).

\subsubsection{Robust multiperiod poverty comparisons for the two- pe- riod case}

In the following we first show empirically how to test for robustness to poverty lines. In this case the arbitrary poverty line is assumed to be constant across the $n$ periods. We then show how to test for robustness to the aggregation procedure by using different poverty lines across periods. To keep the exposition simple and short the empirical illustration will primarily focus on first order stochastic dominance tests using the intersection definition of poverty.

\section{Robustness to poverty lines}

To analyze the robustness to the poverty line we use three waves of the Peruvian household panel data and consider the time spans 1998 to 1999 and 1999 to 2000. According to equation 1.13, for order stochastic poverty comparisons can be made by testing for significant differences between the dominance surface of 1998/99 and the dominance surface of 1999/2000. Testing robustness to the poverty line implies testing all points on the bisector between income in period 1 and income in period 2. Figure 1.2 shows the dominance surface of the first time span 19981999. The $x$ and $y$ axes measure income (or more precisely household expenditure per capita per day) at the beginning (1998) and the end (1999) of the time span. Expenditures are expressed in 2002 US\$ PPP equivalents. The third axis measures the cumulative share of individuals who are below the points defined in the $(x, y)$ domain.

Figure 1.3 shows the difference between the dominance surfaces of the time spans 1999/98 and 1999/2000. The relevant points can be found on the bisector of the graph, since we are testing only robustness to the poverty line (i.e., $z_{1}=z_{2}$ ). The figure shows that for very low incomes, multiperiod poverty was higher in the first than in the second time span for all poverty indices belonging to the class $\Xi_{1,1}$. However, as we increase the poverty line, we find that the cumulative share of people having had an income below that poverty line increases faster and that multiperiod poverty becomes higher for the second time span. This is a very 
Figure 1.2: Poverty in Peru: Dominance Surface of the Time Span 1998/99

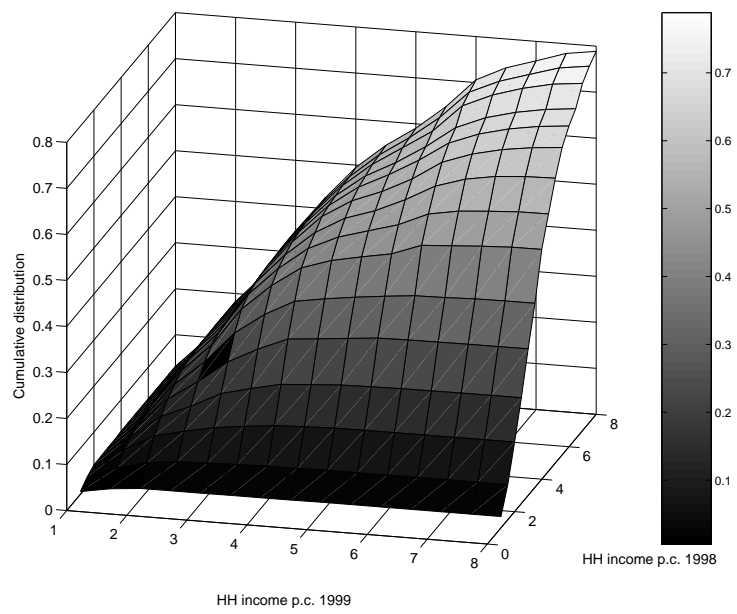

Income: Household income per capita per day in PPP US\$

Source: Authors' calculations based on ENAHO

interesting result because it highlights the importance of conducting dominance tests in this context. It can be seen even more clearly in Table 1.1.

The vertical axis in Table 1.1 shows income at the beginning of the time spans and the horizontal axis at the end of the time spans. The value ' 1 ' indicates a significant positive difference, i.e., 1999/2000 dominates 1998/99. '0' means an insignificant difference, while ' -1 ' indicates a significant negative difference, i.e., 1998/99 dominates 1999/2000. Actually, we should check for poverty dominance at every possible point on this bisector, i.e. at every possible poverty line (e.g., $\$ 1, \$ 1.01, \$ 1.02$, etc.). However, to keep the presentation simple and transparent, we abstained from such a detailed analysis and report results only at all poverty lines that are multiples of $\$ 0.5$. Again, the table demonstrates the relevance of our approach. Relying on the $\$ 1$ poverty line, one can conclude that 'chronic' poverty, i.e. individuals who are under the poverty line in both periods constituting a time span, would have fallen from the first to the second time span because there were more individuals with less than \$1 in 1998 and 1999 than in 1999 and 2000. However, if we rely on the $\$ 2$ poverty line, dominance does not hold anymore given the insignificant differences between the surfaces. Finally, if we rely on the $\$ 3$ poverty line, one can conclude that chronic poverty has risen from the first to the second time span. Thus, any conclusion about poverty orderings relies heavily on the poverty line chosen. In other words, to state that 'chronic' poverty Johannes Gräb - 978-3-653-00480-9 
Figure 1.3: Poverty in Peru: Difference in Dominance Surfaces (1998/99 1999/2000)

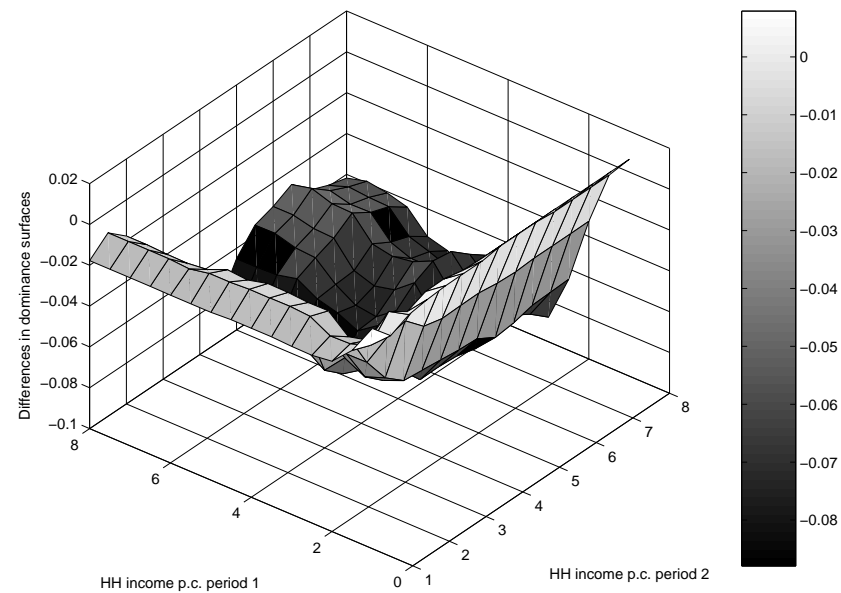

Income: Household income per capita per day in PPP US\$

Source: Authors' calculations based on ENAHO

Table 1.1: Poverty in Peru - Difference in Dominance Surfaces (1998/99 1999/2000)

\begin{tabular}{lccccccccc}
\hline \hline $\begin{array}{l}\text { Income } \\
\text { period 1 }\end{array}$ & & \multicolumn{7}{c}{ Income period 2 } \\
\hline & 1.0 & 1.5 & 2.0 & 2.5 & 3.0 & 3.5 & 4.0 & 4.5 & 5.0 \\
\hline 1.0 & 1 & & & & & & & & \\
1.5 & & 0 & & & & & & & \\
2.0 & & & 0 & & & & & & \\
2.5 & & & & 0 & & & & & \\
3.0 & & & & & -1 & & & & \\
3.5 & & & & & & -1 & & & \\
4.0 & & & & & & & -1 & & \\
4.5 & & & & & & & & -1 & \\
5.0 & & & & & & & & & -1 \\
\hline \hline
\end{tabular}

Income: Household income per capita per day in PPP US\$; 1 indicates that the $1998 / 99$ surface was significantly above the 1999/2000 surface, -1 indicates the opposite, 0 indicates no significant difference. Significance level: $5 \%$

Source: Authors' calculations based on ENAHO 
(as defined here) has changed significantly from one time span to another, one has first to define an appropriate maximum poverty line and then check whether poverty dominance holds at every possible poverty line up to this maximum.

\section{Robustness to Aggregation Procedures}

Robustness to the aggregation procedure seems to be equally important since the weights attributed to different periods are often arbitrary chosen. 'Time discounting', for instance, might appear to be the most appropriate weighting scheme for economists. However, it is empirically very difficult to obtain a reliable and precise value for consumers' discount rates. One therefore needs to be sure that the poverty ordering is robust against alternative weights in a reasonable range. Variations in the discount rate mean changes in the aggregation procedure across periods within a time span. Again, as mentioned above, we chose here a very simple way in attributing different weights to different periods. We simply apply different poverty lines to period 1 and period 2 within each time span. In other words, applying a higher poverty line in the second period than in the first period has the same effect than applying a discount rate to period 2 poverty. As will be demonstrated now (if the time spans under consideration do not overlap) our methodology simultaneously ensures robustness to poverty lines and aggregation procedures. Moreover, it ensures of course also robustness to a wide range of poverty measures.

We compare the time span 1998/1999 with the time span 2000/2001. In contrast to the procedure illustrated above, now one has not only to check for significant differences between the two surfaces at the bisector but at all points below and above the bisector up to a reasonable maximum poverty line. This becomes clear when looking at Figure 1.4 and Table 1.2. Figure 1.4 shows the difference between the two dominance surfaces. A robust poverty ordering would require that one surface is above the other surface at all points up to a reasonable maximum poverty line. This is obviously not the case here. Table 1.2 illustrates this further. Given the many ' 0 ' $s$ ' in the grid of test points, it is clear that poverty dominance cannot be established for any reasonable set of poverty lines in any aggregation procedure.

To underline the economic relevance of our approach, we now show the specific outcomes of weighting period 1 and period 2 differently. We consider poverty orderings $\mathbf{D}$ which are robust for a broad class of poverty measures, $P(F ; z ; r)$ and a large range of poverty lines, $z \in Z$ and discount rates, $r \in R$. Hence, we rely on a poverty index $P$ that assesses the degree of poverty, given a two-period distri- 
Figure 1.4: Poverty in Peru: Difference in Dominance Surfaces (1998/99 2000/01)

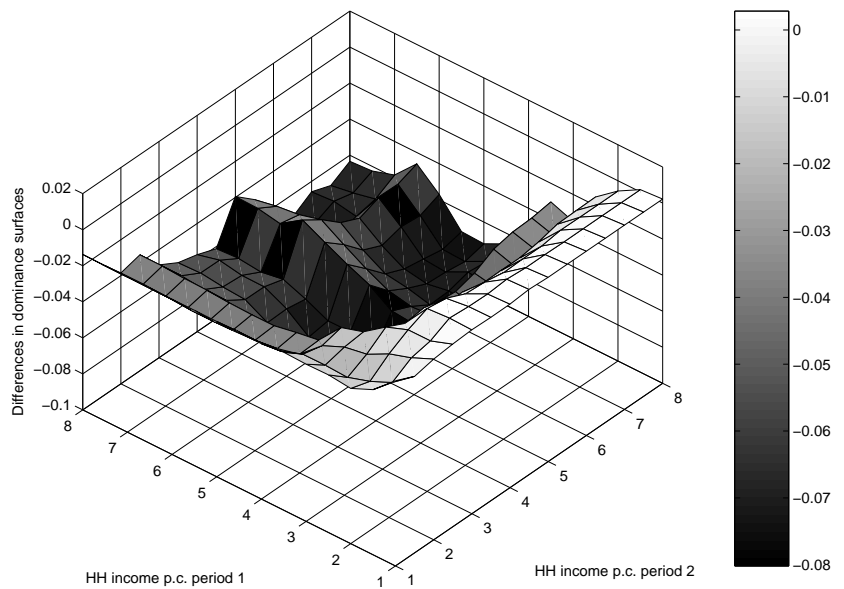

Income: Household income per capita per day in PPP US\$

Source: Authors' calculations based on ENAHO

Table 1.2: Poverty in Peru - Difference in Dominance Surfaces (1998/99 2000/01)

\begin{tabular}{lccccccccc}
\hline $\begin{array}{l}\text { Income } \\
\text { period 1 }\end{array}$ & \multicolumn{10}{c}{ Income period 2 } \\
\hline & 1.0 & 1.5 & 2.0 & 2.5 & 3.0 & 3.5 & 4.0 & 4.5 & 5.0 \\
\hline 1.0 & $\mathbf{0}$ & 0 & 0 & 0 & 0 & 0 & 0 & 0 & 0 \\
1.5 & 0 & $\mathbf{0}$ & 0 & 0 & 0 & 0 & 0 & 0 & 0 \\
2.0 & -1 & 0 & $\mathbf{0}$ & 0 & 0 & 0 & 0 & 0 & 0 \\
2.5 & 0 & 0 & 0 & $\mathbf{0}$ & 0 & 0 & 0 & 0 & 0 \\
3.0 & 0 & 0 & 0 & 0 & $\mathbf{0}$ & 0 & -1 & -1 & -1 \\
3.5 & 0 & 0 & 0 & 0 & 0 & $\mathbf{- 1}$ & -1 & -1 & 0 \\
4.0 & 0 & -1 & -1 & 0 & -1 & -1 & $\mathbf{- 1}$ & -1 & -1 \\
4.5 & 0 & -1 & -1 & 0 & -1 & -1 & -1 & $\mathbf{- 1}$ & -1 \\
5.0 & 0 & -1 & -1 & -1 & -1 & -1 & -1 & -1 & $\mathbf{- 1}$ \\
\hline \hline
\end{tabular}

Income: Household income per capita per day in PPP US\$ 1 indicates that the 1998/99 surface was significantly above the $2000 / 01$ surface, -1 indicates the opposite, 0 indicates no significant difference. Significance level: $5 \%$

Source: Authors' calculations based on ENAHO 
bution $F\left(y_{1}, y_{2}\right)$ when the poverty line is $z$ and the discount factor of subsequent periods to the first period of a given time span is $r$. Therefore, we state that:

$$
\begin{gathered}
F \mathbf{D}_{\mathbf{1}} G \forall P \in \Xi_{1,1}, z \in\left[0, z^{\max }\right], r \in\left[0, r^{\max }\right] \\
\Longleftrightarrow F(z, z(1+r))-G(z, z(1+r))<0 \forall z \in\left[0, z^{\max }\right], r \in\left[0, r^{\max }\right]
\end{gathered}
$$

where $F \mathbf{D}_{1} G$ means that multiperiod poverty is lower over time span $T_{a}$ than over time span $T_{b}$ with respect to all poverty indices belonging to the class $\Xi_{1,1}$, all (time-constant) poverty lines within the range $\left[0, z^{\max }\right]$ and any weighting factor in the range $R$ to discount incomes observed in later periods to the first period constituting a time span.

To illustrate this methodology, we consider the comparison of the time spans 1998/99 and 2000/2002. The two time spans are of different length, such that discounting to the present may be important. ${ }^{6}$ The results are shown in Figure 1.5 and Table 1.3. Table 1.3 has two dimensions. The first dimension corresponds to income, and the second corresponds to the discount rate used. That means that each cell corresponds to one point of the bisector between income in the first and second period of each time span, where income in the second period is discounted by the factor $(1+r)^{-n}$, where $n$ is the length of the respective time span measured in years. For instance, the ' 1 ' in the sixth column of the first row means that if incomes of period 2 in each time span are discounted by a factor 1.05 per year, multiperiod poverty was significantly higher in 1998/99 than in 2000/01. As before, we check at a grid of test points for significant differences of the bisectors for a large range of discount rates and poverty lines. Overall, Table 1.3 shows that in this comparison, $1998 / 99$ vs. $2000 / 2002$, poverty dominance does hold up to a poverty line of $\$ 1.2$ and a discount rate of $r=0.05$, but not beyond.

\section{Comparisons across socio-economic groups}

Another meaningful example for our proposed concept is to compare multiperiod poverty across groups, e.g. socioeconomic categories, within a country. Comparing poverty of employees in the formal private sector with poverty of selfemployed individuals in the informal sector based on multiperiod stochastic dominance could yield different findings than a simple comparison on cross-section comparisons or multi-period average based comparisons. As before, differing results may occur depending on the chosen poverty line and the time-discount rate.

This is now illustrated for Indonesia and the time span 1993/1997. We ask whether intersection poverty was more severe for self-employed than for private

\footnotetext{
${ }^{6}$ One might also argue that past poverty may be more important than present poverty. Hence, it could also be useful to consider negative instead of positive discount rates. 
Figure 1.5: Poverty in Peru: Difference in Dominance Surfaces (1998/99 2000/02)

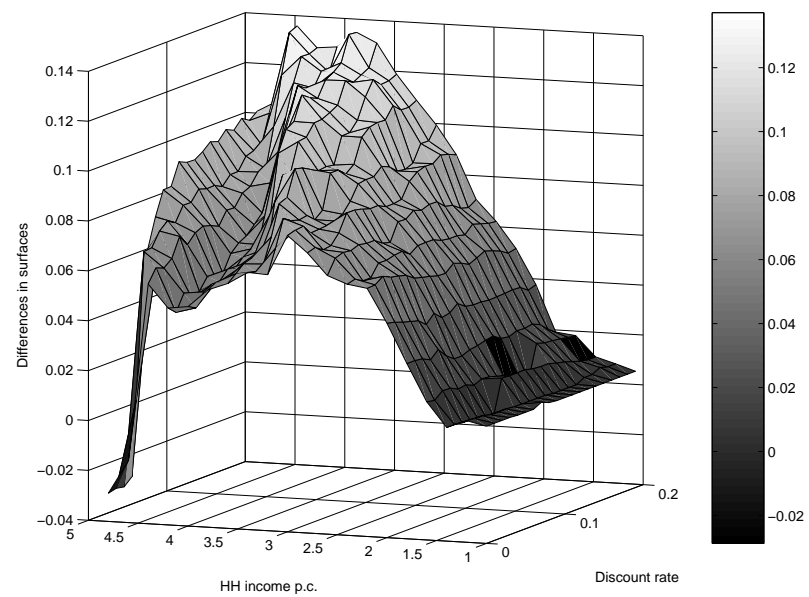

Income: Household income per capita per day in PPP US\$

Source: Authors' calculations based on ENAHO

Table 1.3: Poverty in Peru - Difference in Dominance Surfaces (1998/99 2000/02)

\begin{tabular}{cccccccccccccccc}
\hline \hline Inc. & \multicolumn{11}{c}{ Discount rate of period 2} \\
\hline & .0 & .01 & .02 & .03 & .04 & .05 & .06 & .07 & .08 & .09 & .1 & .11 & .12 & .13 & .14 \\
\hline 1.0 & 1 & 1 & 1 & 1 & 1 & 1 & 1 & 1 & 1 & 1 & 1 & 1 & 1 & 1 & 1 \\
1.2 & 1 & 1 & 1 & 1 & 1 & 1 & 0 & 0 & 0 & 0 & 0 & 0 & 0 & 0 & 0 \\
1.4 & 0 & 0 & 0 & 0 & 0 & 0 & 0 & 1 & 1 & 1 & 1 & 1 & 1 & 1 & 1 \\
1.6 & 0 & 0 & 0 & 0 & 0 & 0 & 0 & 0 & 0 & 0 & 0 & 0 & 0 & 0 & 1 \\
1.8 & 0 & 0 & 0 & 0 & 0 & 0 & 0 & 0 & 0 & 0 & 0 & 0 & 0 & 0 & 0 \\
2.0 & 1 & 1 & 1 & 1 & 1 & 1 & 1 & 1 & 1 & 1 & 1 & 1 & 1 & 1 & 1 \\
2.2 & 1 & 1 & 1 & 1 & 1 & 1 & 1 & 1 & 1 & 1 & 1 & 1 & 1 & 1 & 1 \\
2.4 & 1 & 1 & 1 & 1 & 1 & 1 & 1 & 1 & 1 & 1 & 1 & 1 & 1 & 1 & 1 \\
2.6 & 1 & 1 & 1 & 1 & 1 & 1 & 1 & 1 & 1 & 1 & 1 & 1 & 1 & 1 & 1 \\
2.8 & 1 & 1 & 1 & 1 & 1 & 1 & 1 & 1 & 1 & 1 & 1 & 1 & 1 & 1 & 1 \\
3.0 & 1 & 1 & 1 & 1 & 1 & 1 & 1 & 1 & 1 & 1 & 1 & 1 & 1 & 1 & 1 \\
3.2 & 1 & 1 & 1 & 1 & 1 & 1 & 1 & 1 & 1 & 1 & 1 & 1 & 1 & 1 & 1 \\
3.4 & 1 & 1 & 1 & 1 & 1 & 1 & 1 & 1 & 1 & 1 & 1 & 1 & 1 & 1 & 1 \\
3.6 & 0 & 0 & 0 & 0 & 0 & 1 & 1 & 1 & 1 & 1 & 1 & 1 & 1 & 1 & 1 \\
\hline \hline
\end{tabular}

Income: Household income per capita per day in PPP US\$; 1 indicates that the 1998/99 surface was significantly above 2000/02 surface, -1 indicates the opposite, 0 indicates no significant difference. Significance level: 5\%; Source: Authors' calculations based on ENAHO 
Table 1.4: Poverty in Indonesia - Differences in dominance surfaces (Selfemployed - Private sector)

\begin{tabular}{lcccccccccccc}
\hline \hline $\begin{array}{l}\text { Income } \\
\text { period 1 }\end{array}$ & \multicolumn{10}{c}{ Income period 2 } \\
\hline & 1.25 & 1.5 & 1.75 & 2.0 & 2.25 & 2.5 & 2.75 & 3.0 & 3.25 & 3.5 & 3.75 & 4.0 \\
\hline 1.25 & $\mathbf{1}$ & 0 & 0 & 1 & 1 & 1 & 1 & 0 & 0 & 0 & 0 & 0 \\
1.5 & 1 & $\mathbf{1}$ & 1 & 1 & 1 & 1 & 1 & 1 & 1 & 1 & 1 & 1 \\
1.75 & 1 & 1 & $\mathbf{1}$ & 1 & 1 & 1 & 1 & 1 & 1 & 1 & 1 & 1 \\
2.0 & 1 & 1 & 1 & $\mathbf{1}$ & 1 & 1 & 1 & 1 & 1 & 1 & 1 & 1 \\
2.25 & 1 & 1 & 1 & 1 & $\mathbf{1}$ & 1 & 1 & 1 & 1 & 1 & 1 & 1 \\
2.5 & 1 & 1 & 1 & 1 & 1 & $\mathbf{1}$ & 1 & 1 & 1 & 1 & 1 & 1 \\
2.75 & 1 & 1 & 1 & 1 & 1 & 1 & $\mathbf{1}$ & 1 & 1 & 1 & 1 & 1 \\
3.0 & 1 & 1 & 1 & 1 & 1 & 1 & 1 & $\mathbf{1}$ & 1 & 0 & 0 & 0 \\
3.25 & 1 & 1 & 1 & 1 & 1 & 1 & 1 & 0 & $\mathbf{1}$ & 0 & 0 & 0 \\
3.5 & 1 & 1 & 1 & 1 & 1 & 1 & 0 & 0 & 0 & $\mathbf{0}$ & 0 & 0 \\
3.75 & 1 & 1 & 1 & 1 & 1 & 1 & 1 & 0 & 0 & 0 & $\mathbf{0}$ & 0 \\
4.0 & 1 & 1 & 1 & 1 & 1 & 1 & 1 & 0 & 1 & 0 & 0 & $\mathbf{0}$ \\
\hline \hline
\end{tabular}

Income: Household income per capita per day in PPP US\$; 1 indicates that the 1993/97 surface of the self-employed was significantly above the 1993/1997 surface of the private sector employees, -1 indicates the opposite, 0 indicates no significant difference. Significance level: $5 \%$

Source: Authors' calculations based on ENAHO

sector employees, or vice versa, regardless of the chosen poverty line and aggregation procedure. The results are displayed in Table 1.4. Since there are only few private sector employees with an income below $\$ 1$ per person and day, the grid starts at the $\$ 1.25$ poverty line. Ignoring the issue of the aggregation procedure, the findings demonstrate poverty dominance of private sector employees over selfemployed up to a maximum poverty line of $\$ 3.25$. No matter what poverty line up to a poverty line of $\$ 3.25$ is chosen, one finds more self-employed individuals below the poverty line. If discounting is introduced, this result can be approved almost over the entire grid, except for choosing the $\$ 1.25$ poverty line. If the $\$ 1.25$ poverty line is chosen, discounting income in 1997 to the present value of 1993 could render the differences between the dominance surfaces insignificant as shown by the two ' 0 's' in line 1 . However for any other poverty line between $\$ 1.5$ and $\$ 3$ poverty dominance holds regardless of the applied discount rate.

\subsubsection{Robust multiperiod relative poverty comparisons for the two-period case within and across countries}

We now apply our concept to relative poverty comparisons. To illustrate the idea of relative poverty, consider a household that has experienced a significant increase in income from one period to another and thus moved out of poverty from Johannes Gräb - 978-3-653-00480-9 
an absolute perspective. If the income of almost all households in the region has risen in a similar way, this household might still be poor from a relative perspective, i.e., the poverty gap to the median did not decline. Accordingly, people are referred to as 'chronically poor' in relative terms if their income, measured as a ratio of the median income, stays below a given proportion for consecutive years.

To test for differences in relative poverty between two time spans, we standardize household expenditures by a relative poverty line $\tilde{z}$, i.e., $\tilde{y}=y / \tilde{z}$. We choose $\tilde{z}=50 \%$ of median income. ${ }^{7}$ Accordingly, a relative income of 1 , for example, means that the individual's income is exactly half of the median income.

To illustrate the concept of relative multiperiod poverty, we compare two time spans in Indonesia, namely the time spans 1993/97 and 1997/2000. The difference in relative poverty between these two time spans is presented in Figure 1.6 (note that incomes are standardized to $50 \%$ of the median income, i.e. a value of 0.8 corresponds to $40 \%$ of the median). The $x$ and $y$ axes measure relative income, $\tilde{y}$, at the beginning and the end of the time spans. The figure does not show any systematic pattern. This is supported by Table 1.5, which shows the grid of test points. Here the 0 in the third row of the third column, for example, means that the share of individuals who had less than $50 \%$ of the median income $(\tilde{y}=1)$ did not significantly change between the time spans 1993/97 and 1997/2000. Hence, no conclusions about changes in multiperiod poverty can be drawn.

Our concept of relative poverty orderings is also applicable to cross-country comparisons. Absolute poverty comparisons using some agreed international poverty line are interesting if countries have comparable and rather low living standards. But for countries with very different living standards or for very rich countries, relative poverty might be more relevant. To illustrate this, we now compare Peru to Indonesia. Peru has a median income of $4.7 \$$ PPP and Indonesia of $3.7 \$$ PPP per person per day. For these two countries, we consider the time span 1997/2000 with income observations in 1997 and 2000 for each.

Table 1.6 shows the matrix of test points of differences of the two-period poverty surfaces ('Peru minus Indonesia'). Relative poverty is higher in Peru. Even though dominance cannot be established over the entire domain, the maximum poverty set for relative dynamic poverty is wide enough to conclude dominance. The proportion of poor individuals is higher in Peru no matter what 'reasonable' relative poverty line or aggregation procedure is chosen.

${ }^{7}$ Note that it does not matter which share of the median is used as poverty line.

Johannes Gräb - 978-3-653-00480-9 
Figure 1.6: Relative poverty in Indonesia: Difference in Dominance Surfaces (1993/97 - 1997/2000)

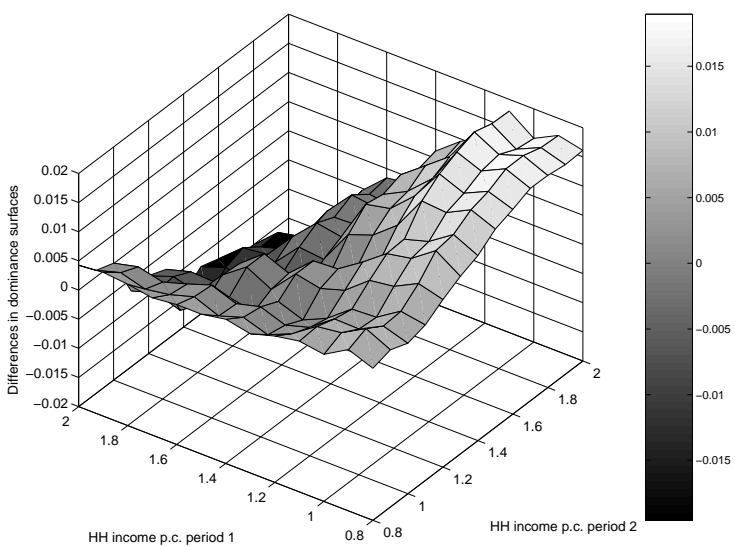

Income: Household income per capita per day in PPP US\$

Source: Authors' calculations based on IFLS

Table 1.5: Relative Poverty in Indonesia - Difference in Dominance Surfaces (1993/97 - 1997/2000)

\begin{tabular}{lccccccccccccc}
\hline $\begin{array}{l}\text { Income } \\
\text { period } 1\end{array}$ & \multicolumn{10}{c}{ Income period 2} \\
\hline & 0.8 & 0.9 & 1.0 & 1.1 & 1.2 & 1.3 & 1.4 & 1.5 & 1.6 & 1.7 & 1.8 & 1.9 & 2.0 \\
\hline 0.8 & $\mathbf{1}$ & 1 & 0 & 0 & 0 & 0 & 0 & 1 & 1 & 1 & 1 & 1 & 1 \\
0.9 & 1 & $\mathbf{0}$ & 0 & 0 & 0 & 0 & 0 & 1 & 1 & 1 & 1 & 0 & 0 \\
1.0 & 0 & 0 & $\mathbf{0}$ & 0 & 0 & 0 & 0 & 0 & 0 & 0 & 0 & 0 & 0 \\
1.1 & 0 & 0 & 0 & $\mathbf{0}$ & 0 & 0 & 0 & 0 & 0 & 0 & 0 & 0 & 0 \\
1.2 & 0 & 0 & 0 & 0 & $\mathbf{0}$ & 0 & 0 & 0 & 0 & 0 & 0 & 0 & 0 \\
1.3 & 0 & 0 & 0 & 0 & 0 & $\mathbf{0}$ & 0 & 0 & 0 & 0 & 0 & 0 & 0 \\
1.4 & 0 & 0 & 0 & 0 & 0 & 0 & $\mathbf{0}$ & 0 & 0 & 0 & 0 & 0 & 0 \\
1.5 & 0 & 0 & 0 & 0 & 0 & 0 & 0 & $\mathbf{0}$ & 0 & 0 & 0 & 0 & 0 \\
1.6 & 0 & 0 & 0 & 0 & 0 & 0 & 0 & 0 & $\mathbf{0}$ & 0 & 0 & 0 & 0 \\
1.7 & 0 & 0 & 0 & 0 & 0 & 0 & 0 & 0 & 0 & $\mathbf{0}$ & 0 & 0 & 0 \\
1.8 & 0 & 0 & 0 & 0 & 0 & 0 & 0 & 0 & 0 & 0 & $\mathbf{0}$ & 0 & 0 \\
1.9 & 0 & 0 & 0 & 0 & 0 & 0 & 0 & 0 & 0 & 0 & 0 & $\mathbf{0}$ & 0 \\
2.0 & 0 & 0 & 0 & 0 & 0 & 0 & 0 & 0 & 0 & 0 & 0 & 0 & $\mathbf{0}$ \\
\hline \hline
\end{tabular}

Income is household income per capita per say in US\$, standardized by a relative poverty line, $\tilde{z}=$ $50 \%$ of median income: Income $=$ Income $/ \tilde{z} ; 1$ indicates that the 1993/97 surface was significantly above the 1997/2000 surface, -1 indicates the opposite, 0 indicates no significant difference. Significance level: 5\%; Source: Authors' calculations based on IFLS 
Table 1.6: Relative Poverty in Peru and Indonesia - Difference in Dominance Surfaces Peru (1997/2000) - Indonesia (1997/2000)

\begin{tabular}{lccccccccccccc}
\hline $\begin{array}{l}\text { Income } \\
\text { period } 1\end{array}$ & \multicolumn{10}{c}{ Income period 2} \\
\hline & 0.8 & 0.9 & 1.0 & 1.1 & 1.2 & 1.3 & 1.4 & 1.5 & 1.6 & 1.7 & 1.8 & 1.9 & 2.0 \\
\hline 0.8 & $\mathbf{1}$ & 1 & 1 & 1 & 1 & 1 & 1 & 1 & 1 & 1 & 1 & 1 & 1 \\
0.9 & 1 & $\mathbf{1}$ & 1 & 1 & 1 & 1 & 1 & 1 & 1 & 1 & 1 & 1 & 1 \\
1.0 & 1 & 1 & $\mathbf{1}$ & 1 & 1 & 1 & 1 & 1 & 1 & 1 & 1 & 1 & 1 \\
1.1 & 1 & 1 & 1 & $\mathbf{1}$ & 1 & 1 & 1 & 1 & 1 & 1 & 1 & 1 & 1 \\
1.2 & 1 & 1 & 1 & 1 & $\mathbf{1}$ & 1 & 1 & 1 & 1 & 1 & 1 & 1 & 1 \\
1.3 & 1 & 1 & 1 & 1 & 1 & $\mathbf{1}$ & 1 & 1 & 1 & 1 & 1 & 1 & 1 \\
1.4 & 1 & 1 & 1 & 1 & 1 & 1 & $\mathbf{1}$ & 1 & 1 & 1 & 1 & 1 & 1 \\
1.5 & 1 & 1 & 1 & 1 & 1 & 1 & 1 & $\mathbf{1}$ & 1 & 1 & 1 & 1 & 1 \\
1.6 & 1 & 1 & 1 & 1 & 1 & 1 & 1 & 1 & $\mathbf{1}$ & 1 & 1 & 0 & 1 \\
1.7 & 1 & 1 & 1 & 1 & 1 & 1 & 1 & 1 & 1 & $\mathbf{0}$ & 0 & 0 & 0 \\
1.8 & 1 & 1 & 1 & 1 & 1 & 1 & 1 & 1 & 0 & 0 & $\mathbf{0}$ & 0 & 0 \\
1.9 & 1 & 1 & 1 & 1 & 1 & 0 & 0 & 0 & 0 & 0 & 0 & $\mathbf{0}$ & 0 \\
2.0 & 1 & 1 & 1 & 1 & 1 & 0 & 0 & 0 & 0 & 0 & 0 & 0 & $\mathbf{0}$ \\
\hline \hline
\end{tabular}

Income is household income per capita per say in US\$, standardized by a relative poverty line, $\tilde{z}=50 \%$ of median income: Income $=$ Income $/ \tilde{z} ; 1$ indicates that the Peru surface was significantly above the Indonesia surface, -1 indicates the opposite, 0 indicates no significant difference. Significance level: 5\%; Source: Authors' calculations based on ENAHO and IFLS

\subsubsection{Robust multiperiod poverty comparisons for the $n$-period case}

Obviously, poverty comparisons over two time spans demand panel data over multiple periods. Consequently, the question arises how the time spans under consideration should be constructed if more than two periods are available within each time span. Which period should be the end of the first and the beginning of the second time span? How many periods should constitute a time span? These are very general questions regarding the measurement of multiperiod poverty (or chronic poverty more specifically). Depending on the panel data available, often several different time span constructions are possible, varying in time span length and the number of periods taken into account. This raises the question, for example, whether comparisons should be made with the maximum overlap (e.g., $T_{a}\left[y_{1}, y_{2}, \ldots, y_{n-1}\right]$ vs. $\left.T_{b}\left[y_{2}, y_{3}, \ldots, y_{n}\right]\right)$, without any overlap (e.g., $T_{a}\left[y_{1}, y_{2}, \ldots, y_{n / 2}\right]$ vs. $\left.T_{b}\left[y_{n / 2+1}, y_{n / 2+2}, \ldots, y_{n}\right]\right)$, or with something in between. Depending on these choices, poverty orderings may differ. Thus, beyond robustness to poverty indices, poverty lines and aggregation procedures, one may also require poverty comparisons to be robust to the construction of the time spans. 
To illustrate this, we use five waves of the Peruvian household panel data (1998-2002). To simplify the exposition, we require that in each comparison, the first period of time span $T_{A}$ is 1998 and the last period of time span $T_{B}$ is 2002. We also abstain from making comparisons for different time span lengths. However, all remaining decisions regarding the construction of these time spans are arbitrary and consequently, any poverty ordering may depend on how exactly the construction is carried out. We think, there are at least five different comparisons that make sense from an economic point of view: three where we consider time spans comprising two periods, one where we consider time spans comprising three periods, and one where we consider time spans comprising four periods:

$$
\begin{gathered}
{[1998 ; 2000] \text { vs. }[2000 ; 2002]} \\
{[1998 ; 1999] \text { vs. }[2001 ; 2002]} \\
{[1998 ; 2001] \text { vs. }[1999 ; 2002]} \\
{[1998 ; 1999 ; 2000] \text { vs. }[2000 ; 2001 ; 2002]} \\
{[1998 ; 1999 ; 2000 ; 2001] \text { vs. }[1999 ; 2000 ; 2001 ; 2002]}
\end{gathered}
$$

Given the difficulty in determining which of these five comparisons is most appropriate, dynamic poverty comparisons should be robust to all of them. For example, one can imagine a case in which the $\$ 3$ poverty line is considered to be a reasonable maximum poverty line when comparing poverty dynamics for the time span 1998-2002 in Peru. In this case, the poverty ordering is only considered robust if poverty dominance can be established for every possible poverty line up to the $\$ 3$ poverty line and for every above-mentioned type of construction for the time spans.

Table 1.7 shows the results of such a dominance test. Obviously, according to our proposed methodology, no significant ordering of poverty dynamics can be established for the time span 1998-2002. This is a very interesting result given the large number of $1^{\prime} s$ in Table 1.7. Suppose the objective is to assess chronic poverty for the time span 1998-2002. Using the $\$ 2$ poverty line and comparing the time spans $[1998 ; 1999 ; 2000]$ and $[2000 ; 2001 ; 2002]$ - which might be judged a reasonable comparison at first glance - one would conclude that chronic poverty has fallen. However, taking the time spans [1998;1999] and [2001;2002] shows instead that no conclusion can be drawn. Hence, the poverty ordering depends not only on the chosen poverty line but also on the way the time spans are constructed.

\subsection{Discussion}

In this paper, we presented a concept allowing to undertake multiperiod poverty comparisons over time and space without arbitrarily aggregating income over various years. Inspired by the multidimensional stochastic dominance methodology Johannes Gräb - 978-3-653-00480-9 
Table 1.7: Poverty in Peru - Difference in Dominance Surfaces for Several Construction Modes of Time Spans

\begin{tabular}{lccccc}
\hline \hline Income & $\begin{array}{c}{[98 ; 00]} \\
\text { vs. } \\
{[00 ; 02]}\end{array}$ & $\begin{array}{c}{[98 ; 99]} \\
\text { vs. } \\
{[01 ; 02]}\end{array}$ & $\begin{array}{c}{[98 ; 01]} \\
\text { vs. } \\
{[99 ; 02]}\end{array}$ & $\begin{array}{c}{[98 ; 99 ; 00]} \\
\text { vs. }\end{array}$ & $\begin{array}{c}{[98 ; 99 ; 00 ; 01 ; 02]} \\
\text { vs. } \\
{[99 ; 00 ; 01 ; 02]}\end{array}$ \\
\hline 1 & 0 & 0 & 0 & 0 & 0 \\
1.5 & 0 & 0 & 0 & 0 & 0 \\
2 & 0 & 0 & 1 & 1 & 1 \\
2.5 & 1 & 0 & 1 & 1 & 1 \\
3 & 1 & 0 & 0 & 0 & 1 \\
3.5 & 1 & 0 & 0 & 0 & 0 \\
4 & 0 & 0 & 0 & 0 & 0 \\
\hline \hline
\end{tabular}

Income: Household income per capita per day in US\$; 1 indicates that the earlier surface was significantly above later surface, -1 indicates the opposite, 0 indicates no significant difference. Significance level: 5\%. Source: Authors' calculations based on ENAHO

elaborated by Duclos et al. (2006b), we created $n$-period income surfaces for different time spans. These surfaces were then ordered using dominance tests. Once dominance is established, the poverty ordering is robust to a wide range of poverty indices, to a wide range of poverty lines, and to a wide range of aggregation procedures. Furthermore, we extended our framework to the measurement of relative poverty.

To illustrate our methodology, we compared poverty across time spans in Peru and between Peru and Indonesia. Furthermore, we highlighted some general problems of dynamic poverty comparisons, i.e. how time spans should be constructed, namely which period should be the end of the first and the beginning of the second time span and how many periods should constitute a time span? We dealt with these questions by applying robustness test with respect to various of these possibilities.

However, the approach suggested and the ideas developed in this paper also have their limitations. The most important of these is certainly that all results are based on a sample of expenditures declared by households and that these declarations are generally affected by measurement error, which affects the bivariate distribution $F\left(y_{1}, y_{2}\right)$ (and $n$-variate distribution) much more than the univariate distribution $F(y)$. In fact, many empirical studies show that measurement error is such that the extent of $\beta$-convergence over time is overestimated (see Bound et al. (2001); Breen and Moisio (2004); Grimm (2007)). For our case, this would imply that multiperiod poverty is underestimated. In the absence of information on 'true income' or any instruments, there is not much that can be done about this, but it should be kept in mind when interpreting our results. However, the problem is obviously not specific to our approach but inherent in most approaches to the analysis of poverty dynamics. 


\title{
Essay 2
}

\section{Spatial inequalities explained - Evidence from Burkina Faso}

\begin{abstract}
Empirical evidence suggests that regional disparities in incomes are often very high, that these disparities do not necessarily disappear as economies grow and that these disparities are itself an important driver of growth. We use a novel approach based on multilevel modeling to decompose the sources of spatial disparities in incomes among households in Burkina Faso. We show that spatial disparities are not only driven by the spatial concentration of households with particular endowments but to a large extent also by disparities in community endowments. Climatic differences across regions do also matter, but to a much smaller extent.
\end{abstract}

based on joint work with Michael Grimm. 


\subsection{Introduction}

Empirical evidence suggests that regional disparities in growth and poverty are often very high, that these regional disparities do not necessarily disappear as economies grow and develop and that these disparities are itself often an important driver of the overall performance of an economy. ${ }^{1}$ Often such regional inequalities are closely linked to key policy choices (e.g. trade policy) and patterns of public spending. But in most cases lagging regions also suffer under infrastructure bottlenecks, adverse agroclimatic conditions, import competition and limited scope for non-agricultural activities.

Burkina Faso is one among many Sub-saharan African countries where the regional pattern of living standards is particularly puzzling. Some of the observed inequality can be related to cotton production given that cotton is the main export commodity of the Burkinabe economy. However, despite the cotton boom which Burkina Faso knew in the middle and end of the 1990s, some cotton producing provinces did grow slower than other non-cotton provinces. In particular the traditionally poor and arid North of the country knew a quite good development during that time. Hence, from these observations it is difficult to guess to what extent agro-climatic factors, trade exposure and population structure matter for disparities in the level and change in living standards. Explaining where such disparities come from could help to design development strategies and interventions to reduce them in a cost-effective way.

Standard poverty assessments usually address such issues simply by undertaking a rather descriptive analysis of growth patterns across regions and by performing decompositions of inequality indices by regional units. However, such decompositions make it very difficult to disentangle what is due to heterogeneity in household characteristics and what is due to heterogeneity in area-specific characteristics or endowments. In other words poor areas could simply be poor because households with poor endowments are geographically concentrated.

To deal with this problem, Ravallion and Wodon (1999) relied on two consecutive cross-sections of household survey data for Bangladesh to run separate regressions for each year and for each of the urban and rural sectors. They included a wide range of household characteristics and attributed the remaining part of the observed variance to geographic effects. They then undertake a number of robustness checks to exclude that there is a bias due to omitted household characteristics which are spatially correlated. The authors conclude that there are sizeable spatial differences in the returns to given household characteristics, i.e. the same household might be poor in one but not in the other region.

\footnotetext{
${ }^{1}$ The 'Operationalizing Pro-Poor Growth Project', for instance, which was coordinated by the World Bank and British, French and German donors, shows various cases in point (see Besley and Cord (2007); Grimm et al. (2007)).
} 
Another approach was chosen by Jalan and Ravallion (2002) and later by De Vreyer et al. (2009). They used several waves of panel-data to implement a quasidifferencing method to identify the impact of locally determined geographic and socioeconomic variables on household's consumption growth while removing unobserved household and community fixed effects. These authors find, for rural China and Peru respectively, robust evidence of geographic poverty traps and highlight in particular the socio-economic features of villages and the provision of public goods, such as rural roads, as important area-specific determinants.

Benson et al. (2005) have used alternatively spatially regression and geographically weighted regression techniques to allow regression error terms to be spatially correlated and to assess the degree to which determinants of poverty and the prevalence of poverty vary across space. For rural Malawi the authors find not much evidence for local poverty traps, characterized for instance by low agricultural productivity, and emphasize that the determinants of poverty vary spatially in their effects across the country. However, they find some evidence that regions with more opportunities for non-agricultural earnings and more markets, public infrastructure and services show less poverty.

While all these studies suggest that poverty reduction efforts have to be targeted at the sub-national level, they do not provide a decomposition of the variance in living standards observed within and between spatial units. In this paper we suggest a novel methodology to address this issue. We build a multilevel random coefficient model able to decompose the variance in living standards across four spatial levels; households, communities, provinces and (agro-climatic) regions. ${ }^{2}$ Moreover, our model allows to decompose the variance measured on each level in a component accounting for the variance in level-specific characteristics and components accounting for a sorting of lower-level characteristics across these levels. For instance, the variance in households' living standards between communities might be driven by the variance in community-specific endowments and by a sorting of households with favorable and unfavorable characteristics across communities.

To implement our approach for Burkina Faso, we build a very detailed and exhaustive data set combining household living standard measurement survey data, population census data, agricultural survey data and a number of statistics collected at the provincial level.

The remainder of our paper is organized as follows. In Section 2 we describe spatial inequality and its development over time in Burkina Faso. In Section 3 we present our data and the empirical strategy. In Section 4 we discuss our results. In Section 5 we conclude.

\footnotetext{
${ }^{2}$ Similar techniques have been applied by Bolstad and Manda (2001) and Ecob (1996) to study spatial inequality in child mortality and health.
} 


\subsection{Regional Growth and Inequality in Burkina Faso}

Burkina Faso is one of the poorest countries in the world. GDP per capita is estimated at only PPP US\$ 1,213 and according to the Human Development Index, the country was ranked 176th out of 177 countries (UNDP, 2007). It is a landlocked country in the middle of West-Africa with a population of roughly 13.4 million. It has a very low human capital base and only very few natural resources. The country depends highly on cotton exports, which account for almost 60 percent of total export earnings, as well as on international aid. More than 80 percent of the Burkinabe population lives in rural areas working predominantly in the agricultural sector, which suffers from very limited rainfall and recurrent severe droughts. The country experienced sustained growth with moderate poverty reduction during the last 15 years however accompanied by important variations over time and space (Grimm and Günther (2007)).

If income levels and growth rates as well as poverty shares are compared across Burkina's 13 regions (see Table 2.1), ${ }^{3}$ one can state that the Western regions, where the bulk of cotton is produced - Hauts Bassins, Mouhoun and Cascades - are richer than the remaining regions (abstracting from the two urban centers Ouagadougou and Bobo-Diolassou). However, in terms of growth in the subsequent period, the non-cotton and initially very poor Eastern regions - Sahel, Est and Centre-Nord - performed better than all cotton regions, despite the very favorable development of cotton exports and the widespread belief that cotton exports were the driver of Burkina Faso's growth. In terms of poverty, Hauts-Bassins has still, given its relatively high income level (by Burkinabe standards) moderate poverty without however any significant poverty reduction since 1994. Mouhon, another of the important cotton regions, had ever and has still very high poverty levels. The cotton region Cascade achieved to halve poverty between 1994 and 2003 (Grimm and Günther (2007)).

\footnotetext{
${ }^{3}$ The household survey data is presented in detail in Section 3.
} 


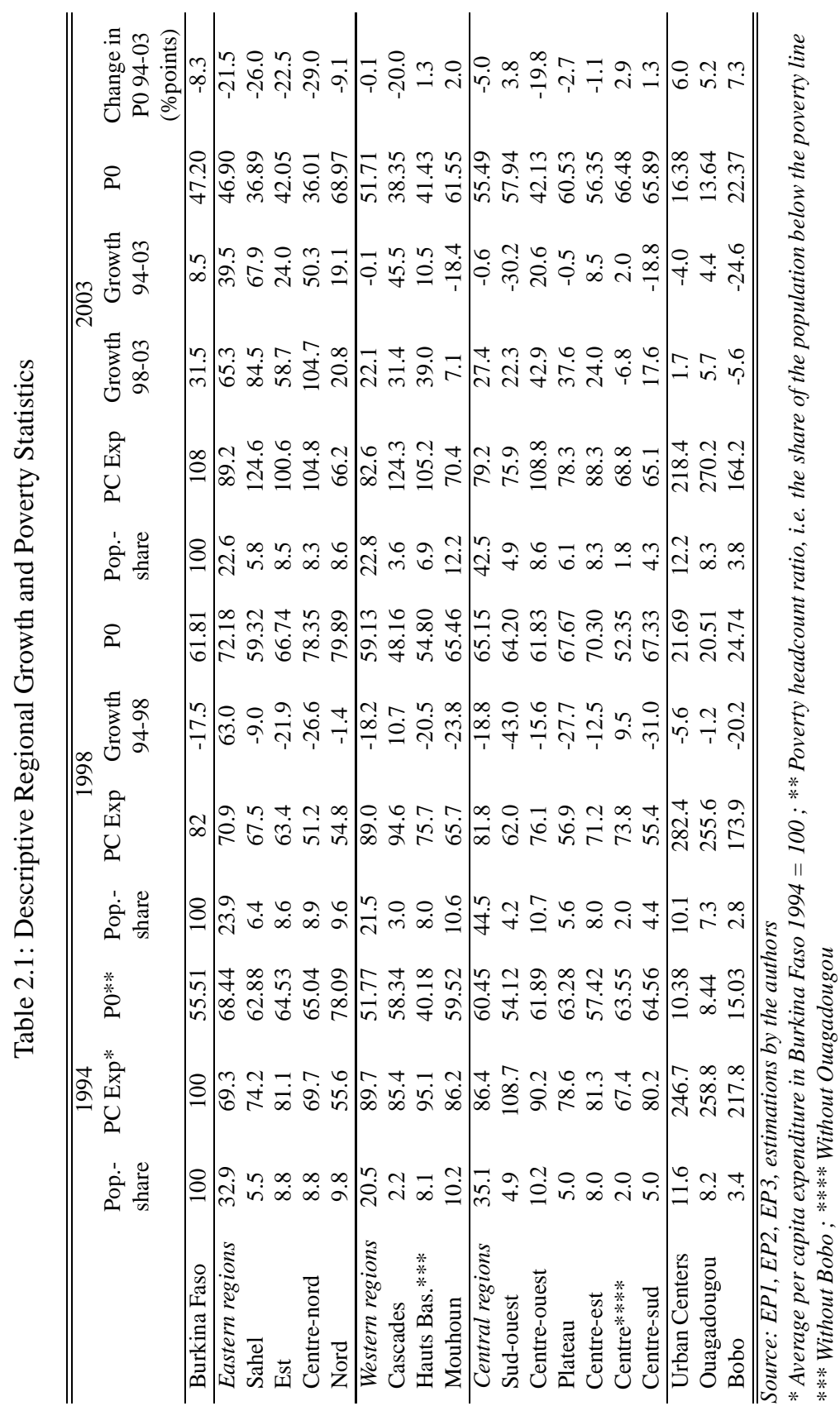


To see if the observed pattern of economic growth and poverty reduction follows a similar pattern on the provincial level, i.e. to see whether provinces in a given region develop similarly, we further disaggregate the data according to Burkina Faso's 45 provinces. The results are presented using maps (Figure 2.1). These maps indicate two important aspects. First, neither does economic growth occur on some widespread regional level nor does there seem to be a high regional concentration of poverty. The intensity of growth and poverty rather varies across provinces over the whole country. Second, the set of provinces with the highest poverty incidence changes over time. Similar to what Benson et al. (2005) have found for rural Malawi, there do not seem to be spatial poverty traps in Burkina Faso.

Figure 2.1: Growth and Poverty Incidence on Provincial Level

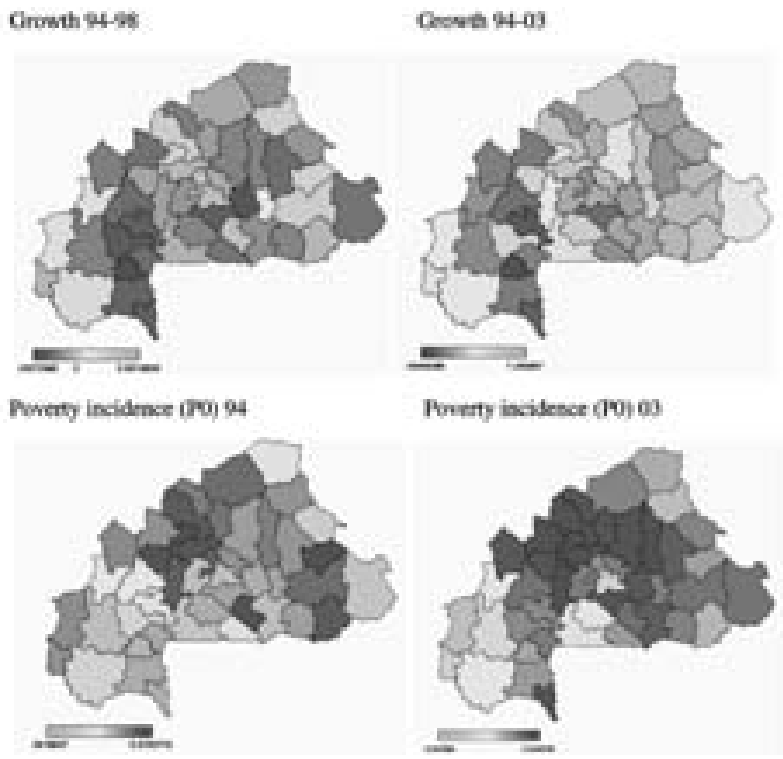

Source: EP1, EP2, EP3, estimations by the authors

If we disaggregate our data further by the 135 districts (Départements) which are covered by the household surveys ${ }^{4}$ and plot household expenditures per capita in 1994 against growth of household expenditures per capita over the period 1994

\footnotetext{
${ }^{4}$ In total Burkina Faso has 301 districts (Départements).
} 
to 2003 , the data suggest $\beta$-convergence in living standards across these local units. However such kind of convergence might be exaggerated if expenditures per capita are measured with error (see e.g., Sala-i-Martin (1996)). Although we provide below some evidence why such convergence could have occurred, we do not find robust empirical evidence for these channels and we cannot rule out that measurement error plays an important role. First, because we do not find evidence for $\sigma$-convergence, which would be immune to the measurement error problem (see e.g., Sala-i-Martin (1996)). Second, we find a much smaller $\beta$ convergence coefficient if we regress the growth rate of expenditures from 1998 to 2003 on expenditure levels in 1994, which again could be sign of measurement error. However one should note that 1998 is a very particular year, since the 1997/98 harvest was affected by a severe drought, even by Burkinabe standards.

Figure 2.2: Convergence in Burkina Faso, initial per capita income and growth on the department level (135 observations), 1994-2003

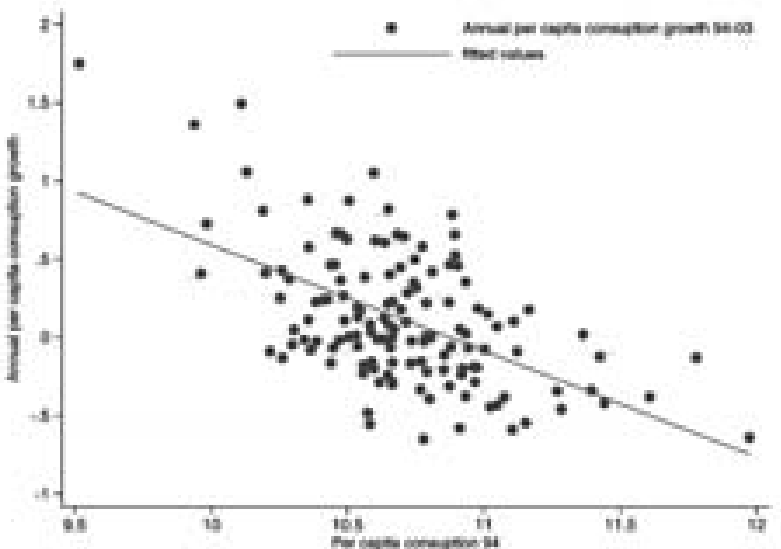

Source: EP1, EP2, EP3, estimations by the authors

Hence, the question arises how income disparities between households and across spatial units can be explained. What is the contribution of the variance in household characteristics and level-specific endowments such as public services, infrastructure and climate? To what extent does the spatial clustering of households play a role? Are the effects of relevant factors similar across spatial units or do they vary significantly across the country? Answers to this kind of questions have not yet been given for Burkina Faso, but seem crucial to appropriately target poverty alleviation strategies. The only study we have found that did research in that direction for the case of Burkina Faso is Bigman et al. (2000). Similar to our study, the authors use a very detailed data set combining information from the 
household, village, district and provincial level and construct a poverty map on the level of villages. From that map the authors conclude that differences in the incidence of poverty among regions are primarily due to differences in agro-climatic conditions, whereas differences in the incidence of poverty among villages within the same region do often reflect past policy biases that led to differences in the quality of roads or public services.

\subsection{Data and Empirical Strategy}

\subsubsection{Data}

Burkina Faso is organized in 13 agro-climatic regions, 45 provinces and 301 districts (départements). It has 26 cities and towns (population $>5,000$ ) and roughly 9,000 villages. According to the last census in 2006 the urbanization rate was about 16 percent and the average population density 48.4 persons per $\mathrm{km}^{2}$. The two major cities are Ouagadougou, the capital, with a population of roughly 1.1 million and Bobo-Dioulasso with a population of about 0.4 million. The third city, Koudougou only has a population of 83.4 thousand. ${ }^{5}$ The variables we use have been collected from a large number of sources and on different levels of that organizational structure. However, it was very difficult to find and get access to data on agro-climatic characteristics, infrastructure and public services and if it existed to match these data to other sources. This seems to be a problem in many of the least developed countries and may explain why only very few attempts have been made so far to analyze the effects of area-specific characteristics on households' living standards.

First, household data is drawn from three nation-wide representative household surveys, the Enquête Prioritaires (EP), conducted in 1994 (EP I), 1998 (EP II) and 2003 (EP III) covering around 8,500 different households in each year. These surveys were conducted by the Institut National de la Statistique et de la Démographie (INSD) with technical and financial support of the World Bank. These surveys contain relatively detailed information on household's socio-demographic characteristics, education, employment, agricultural and non-agricultural activities as well as consumption, income and some assets. ${ }^{6}$

Given the usual low quality of income data in poor rural settings, we use household expenditure per capita as an indicator of households' living standards. Expenditures were deflated over time and space using appropriate price deflators. A critical issue in our study are of course the deflators used to correct for price differences across space. For this purpose we use deflators provided by the INSD

\footnotetext{
${ }^{5}$ Statistics taken from INSD, see http://www.insd.bf.

${ }^{6} \mathrm{~A}$ detailed description of these data sets can be found in Grimm and Günther (2007). Johannes Gräb - 978-3-653-00480-9
} 
in each survey year for Burkina Faso's 13 regions (based on price data collected on 37 different regional markets).

Second, we can draw data on the community (or cluster) level from several sources. Although, except in 1998, the above mentioned household surveys were not linked to any village survey, the questionnaires contain some questions regarding the time needed to reach the next primary and secondary school, the next health center, road, market and drinking water point. In 1998 a specific community survey was added to the household survey which collected further community data for 325 of the 425 communities covered by the survey. Further community variables were constructed simply by aggregating household characteristics at the community level. However, a community panel cannot be constructed because each survey year does not cover exactly the same communities.

Third, data on the size of agricultural production units, fertilizer use and the use of modern production technologies in agriculture are drawn from a yearly agricultural survey called Enquête Agricole. This survey is conducted by the Ministry of Agriculture in collaboration with INSD. Since the data set uses a different survey design than the EPs, we merged the information to the other data sources on the provincial level, the smallest common regional unit. The average size of agricultural production units, fertilizer use and information about modern production technologies are therefore provincial averages.

Fourth, data on agro-climatic conditions such as monthly rainfall for the period 1993-2006 on the provincial level, and monthly minimum and maximum temperatures on the regional level were obtained from the Directorate of Meteorology (Direction de la Météorologie).

Fifth, data on the provision of public services, infrastructure and population densities, also at the provincial level, were obtained from the Ministry of Infrastructure (Direction Génerale de l'Amenagement du Territoire). Note that we do not have any data on project aid, hence the effect of aid will be in the unobservables.

Hence, as stated above, the data set we use is organized in four levels: the household, the community (cluster), the province and the region. Table 2.2 shows all used variables along with their means and standard deviations and their source. 


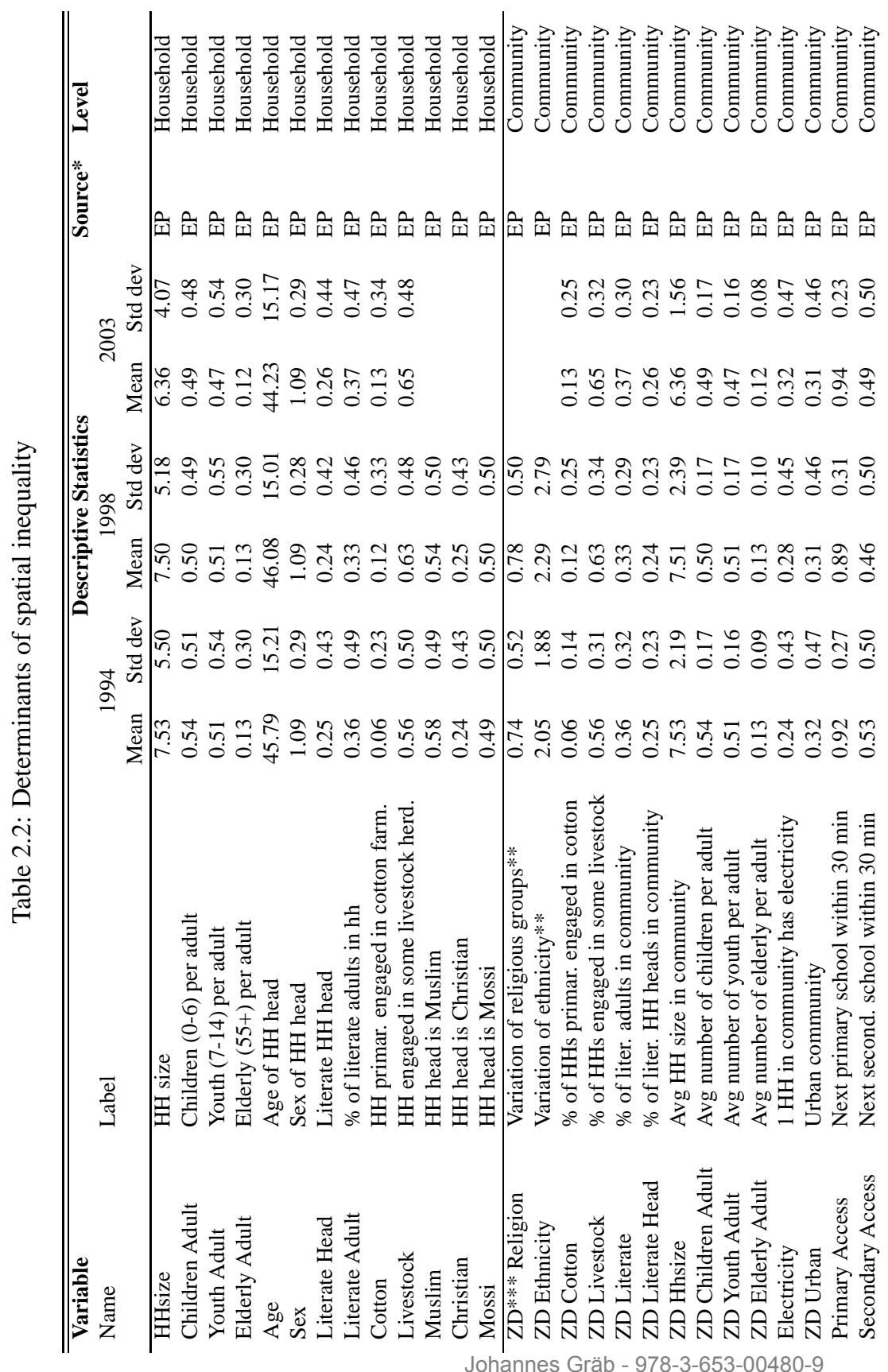




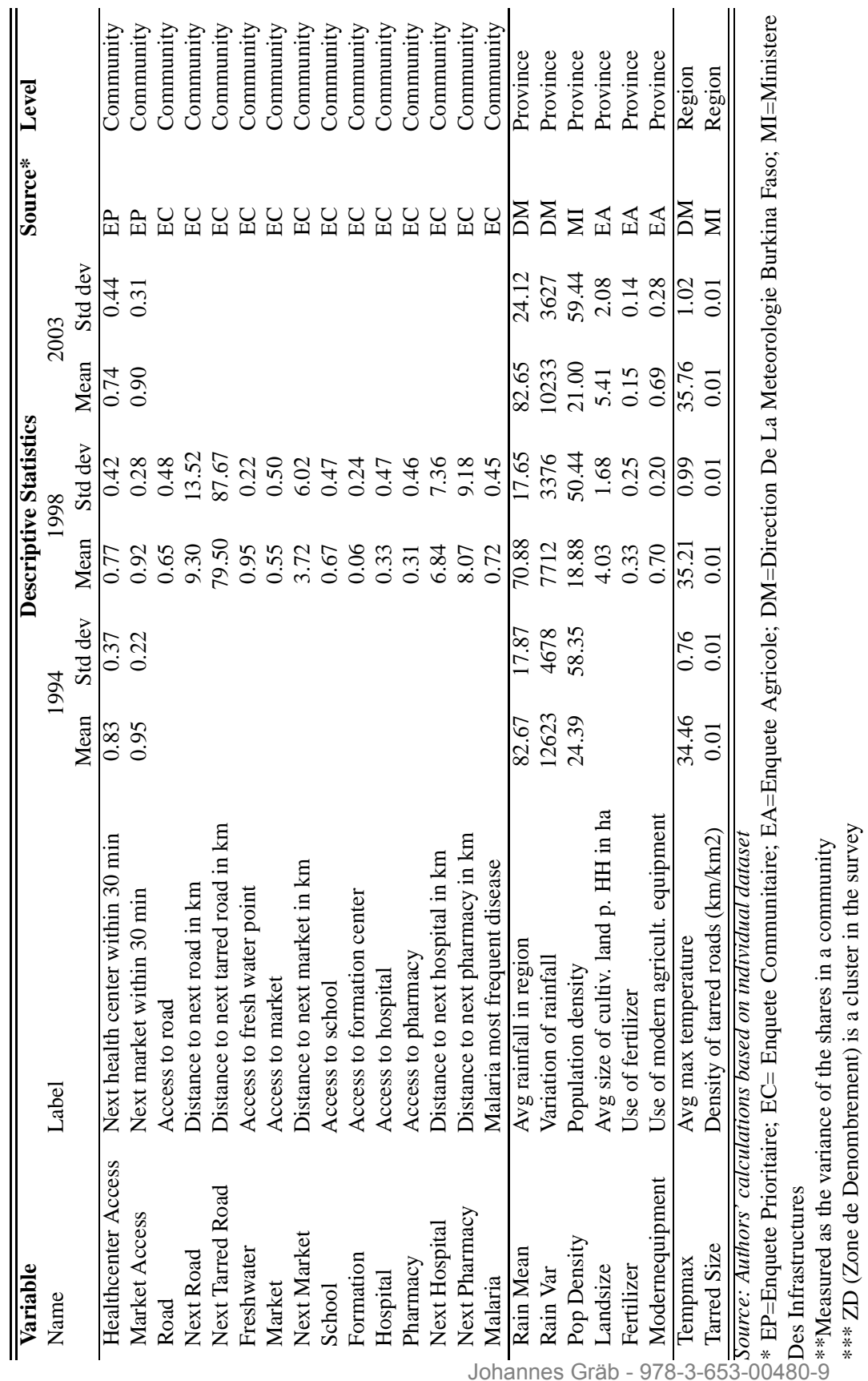




\subsubsection{Empirical Strategy}

To analyze the determinants of income levels and to decompose the variance in income levels across spatial units, we use a multilevel (also hierarchical or mixed) regression model. ${ }^{7}$ Multilevel models are widely used in social science, sociology and health research to specify the effect of social context on individual level outcomes. ${ }^{8}$ Due to the often observed lack of hierarchical data and probably due to the very time consuming estimation procedure, multilevel models are less popular in economics than in these other disciplines. ${ }^{9}$

\section{A multilevel model}

A multilevel model can be best described by beginning with a two level random coefficient model with only one explanatory variable. The idea of the model is, that the regression coefficient on the first level (e.g. households), $i$, is treated as a random variable at the second level (e.g. communities), $j$.

The model equation reads:

$$
Y_{i j}=\beta_{0 j}+\beta_{1 j} X_{i j}+\varepsilon_{i j}
$$

The regression coefficients $\beta_{0 j}$ and $\beta_{1 j}$ can be expressed as:

$$
\begin{aligned}
& \beta_{0 j}=\gamma_{00}+U_{0 j} \\
& \beta_{1 j}=\gamma_{10}+U_{1 j}
\end{aligned}
$$

Equation 2.2 shows that for each unit $j$ on the second level, a specific intercept, $U_{0 j}$, is introduced into the model. These intercepts are however not directly estimated as a fixed coefficient within the model. Multilevel models estimate the variance of these $U_{0 j}$. They are therefore often referred to as random intercepts. Equation 2.3 shows that a specific $\beta$-coefficient, $U_{1 j}$, is introduced allowing the effects associated with the covariates to vary across units on the second level. Since only the variance of these coefficients is estimated, it is referred to as a random coefficient. Models that do only include random intercepts are called random intercept models, while models that include random intercepts and random coefficients are called random coefficient models.

\footnotetext{
${ }^{7}$ For a comprehensive overview of the statistical theory underlying multilevel modeling and of various illustrative applications, see e.g. Goldstein (2003) and Hox (1995)

${ }^{8}$ For a good overview of applications in that area, see DiPrete and Forristal (1994).

${ }^{9}$ Economists rely on these models in particular for out of sample predictions to perform small area estimations, for instance to construct a poverty map (see Elbers et al. (2003) and Jiang and Lahiri (2006)). A paper which deals with causal multilevel models is, for example, Aassve and Arpino (2007).
} 
Finally, the combined model can be expressed as consisting of a fixed part (first term) and a random part (second term): ${ }^{10}$

$$
Y_{i j}=\left(\gamma_{00}+\gamma_{10} X_{i j}\right)+\left(U_{0 j}+U_{1 j} X_{i j}+\varepsilon_{i j}\right)
$$

It is straightforward to extend the model to more than two levels. The model can also be used to check for significant variation of the random intercepts and slope coefficients across units on each level. Moreover, it is possible to analyze the covariance of the random intercepts and slopes.

\section{Strengths of a multilevel model}

Multilevel models offer several advantages over other models. They allow to combine nested data from different sources, to decompose variation across levels and to model the variation of effects across spatial units. In what follows we discuss each of these advantages.

\section{Efficient Estimation}

Since we built our data set using several different and independent data sets, variables are observed on multiple nested levels (see Table 2.2). Clustering stemming from this nested structure requires to account for intra-group correlations. Under the assumption that individuals and households on the same level are more alike than individuals and households from different levels, within group residuals are likely to be correlated. Applying standard OLS regression to nested data leads to a wrong of standard errors and, hence, statistical inference can be wrong. In a multi-level data set the unexplained variance should be decomposed into the variance on all nested levels. This is exactly done by the multilevel model allowing to obtain efficient estimates (see Goldstein (2003)).

\section{Variance partitioning}

In a multilevel random intercept model, the decomposition of the error term allows to assess how much of the total variance is attributable to variation on the different nested levels. Moreover it can be assessed how much of the variance measured on each level is due to the variance in level-specific characteristics and how much is due to sorting of lower-level characteristics across these levels. For instance, the variance in households' living standards between communities might be driven by the variance in community-specific endowments and by a sorting of households with favorable and unfavorable characteristics across communities.

\footnotetext{
${ }^{10}$ Fixed effects are hereafter denoted as coefficients which are directly estimated by the model. For random effects only the variance and its standard error is estimated. 
More precisely, sticking to this two-level example, we can answer the following questions:

1. How much of the total variance in incomes between households is attributable to differences between communities?

2. How much of the variance between communities can be explained by differences in observed household characteristics between theses communities?

3. How much of the variance between communities can be explained by differences of observed community characteristics?

The contribution of the variance at each level to the total variance can be measured with the so-called 'variance partition coefficient', also called the 'intra-class correlation coefficient' ('icc', hereafter), $\rho .{ }^{11}$ Since a multilevel model implicitly assumes errors to be independently distributed across levels, the total variance of the dependent variable can be decomposed as the sum of the variance on each level. If we use again the two-level model as an example, the decomposition of the variance by level reads:

$$
\operatorname{var}\left(Y_{i j} \mid X_{i j}\right)=\operatorname{var}\left(U_{0 j}\right)+\operatorname{var}\left(\varepsilon_{i j}\right)=\sigma_{u_{0}}^{2}+\sigma_{\varepsilon}^{2} .
$$

Accordingly, the icc of the second level can be expressed by:

$$
\rho=\frac{\sigma_{u_{0}}^{2}}{\sigma_{u_{0}}^{2}+\sigma_{\varepsilon}^{2}} .
$$

The intra-class correlation coefficient measures the correlation of the residual of the response variable of households stemming from the same community. A high $\rho$ in equation 2.6 would point to a large impact of the second level, for instance the community, on first level outcomes, i.e. on the level of households.

Finally, the decomposition allows to draw conclusions on the explanatory power of the used covariates with respect to the variation on the different levels (see Borgoni et al. (2002)). For instance, we can answer the question whether the observed spatial pattern in income levels can rather be explained by differences in regional variables, like geographic traits, by differences in community characteristics like access to certain public goods or rather by differences in household characteristics, like household size and education. This is a major conceptual advantage of a multilevel model. If we ran a household income regression with explanatory variables on higher levels, but without a multilevel structure, significant

\footnotetext{
${ }^{11}$ It is called 'intra-class correlation coefficient' since it measures the degree to which observations in the same unit of a given level, e.g. households within a given community, are dependent. 
coefficients of these variables are likely to pick up variation which is at least partly due to omitted household level variables. In contrast, if we introduce a random intercept on each level, we can test the explanatory power of level-specific variables on each level separately. Whenever an introduced variable reduces the variance of the level-specific error term, we can conclude that this variable explains part of the variance in incomes on that level (see Ecob (1996)).

\section{Area-specific returns}

A multilevel model designed as a multilevel random coefficient model (' $\mathrm{RC}$ ' hereafter), allows to take into account a possible variation in the factor coefficients across spatial units. Finding significant variation in the effects of individual characteristics across spatial units suggests that area modifies the association between individual characteristics and income (see Merlo et al. (2005b)). In our case, for instance, it will be interesting to see whether effects associated with education, cotton cultivation or household composition are constant across spatial units.

\section{Covariance structure of random effects}

Finally, the RC model allows us to investigate the covariance structure of the random intercepts and random slope coefficients. For instance, it might be that communities with lower average income levels (a lower intercept) have higher returns associated with education or cotton cultivation. A significant negative correlation, for example, could explain the convergence described in Section 2.

To conclude, based on these methodological considerations, we believe that a multilevel model is particularly suitable to identify the sources of spatial inequalities. Our methodology is capable of decomposing spatial inequality into the contribution of household and area-specific characteristics, of identifying the key spatial determinants of inequality and of tracking variations in returns across space, thereby preserving simultaneously most of the advantages of the methods used by Ravallion and Wodon (1999), Jalan and Ravallion (2002) and Benson et al. (2005). Complementing the geographical analogue of the Oaxaca-Blinder decomposition proposed by Ravallion and Wodon (1999), our decomposition methodology allows to attribute weights to the contribution of the various levels to total inequality. Moreover, in addition to the identification of higher level variable effects on household income, which is done in Jalan and Ravallion (2002) using a GMMtype approach, our model differentiates in principle between significant higher level effects explaining higher level inequality and significant higher level effects just picking up omitted household characteristics. 
Obviously, our methodology also has some drawbacks. In the absence of panel data, we cannot exclude that we run with some of our explanatory variables into endogeneity problems. However, the methodology we propose is just as applicable to panel data as it is to cross sectional data. Multilevel models are also often criticized for inconsistent parameter estimation. ${ }^{12}$ However, we are not particularly focussing on consistent parameter estimation but on variance partitioning. It should be noted moreover, that due to the few observations that we observe per first level unit (maximum 20 households per community), introducing dummy variables for each higher level unit to satisfy the independence assumption would lead to a significant over-parametrization (Lombardía and Sperlich, 2007). Simultaneously, effects of all higher level variables, which are key for our analysis, could not be identified. Thus, we will construct our multilevel model in a way that we can benefit from all the advantages of a multilevel model while using our large data set to control as much as possible for unobserved heterogeneity.

\section{Modeling Strategy}

We use an iterative procedure to estimate the sources of spatial inequality. We start with a multilevel random intercept model (M0), that will not include any covariates. We will then iteratively introduce household level variables (M1), community variables (M2) and provincial and regional variables (M3) into the model. ${ }^{13}$ At each stage, our main concern is about two questions:

1. What are the key characteristics determining per capita income disparities?

2. To what extent are the characteristics responsible for the spatial variation observed on each level?

Finally, we will augment our multilevel model in section 2.4 .5 by allowing coefficients of household characteristics to vary across communities and by modeling the covariances of the random effects on that level (M4). Investigating the variance of the random coefficients and the correlation between random intercepts and slopes, the model can help answer the following questions:

\footnotetext{
${ }^{12}$ Multilevel modeling does only control for unobserved heterogeneity on each level as long as the independence assumption between unobserved characteristics and the regressors holds. In the context of hierarchical data, multilevel models assume area effects to be independent of the covariates and any unobserved individual effects.

${ }^{13}$ Following such an iterative modeling procedure has some drawbacks. Model M1 and M2 may suffer from dependency with observed but non-included higher level variables. Results should therefore - as will be mentioned later on - be interpreted with caution. Alternatively, one could run M3 right after M0. Then, it would be possible to estimate directly the contribution of the set of observed variables of each level to the reduction of each variance component.
} 
3. Does area modify the association between household characteristics and respective outcomes?

4. What might be the explanation for such a modification?

We estimate our model for three points in time: 1994, 1998 and 2003. This will also allow to get some insights into the dynamics of spatial inequality and its determinants. Our full four level random coefficient model reads:

$$
\begin{gathered}
Y_{i j k l}=\left(\gamma_{0000}+\sum_{p=1}^{P} \gamma_{p 000} X_{p i j k l}+\sum_{q=1}^{Q} \gamma_{0 q 00} C_{q j k l}+\sum_{r=1}^{R} \gamma_{00 r 0} P_{r k l}+\sum_{m=1}^{M} \gamma_{000 m} R_{m l}\right) \\
+\left(W_{l}+V_{k l}+U_{j k l}+\sum_{p=1}^{P} U_{p j k l} X_{p i j k l}+\varepsilon_{i j k l}\right)
\end{gathered}
$$

where $i$ stands for households, $j$ for communities, $k$ for provinces and $l$ for regions. $X, C, P$ and $R$ are vectors of household, community, provincial and regional characteristics, respectively. $W_{l}$ is the regional random intercept, $V_{k l}$ the provincial random intercept and $U_{j k l}$ the community random intercept. The models will be estimated using Stata and its implemented mixed model command 'xtmixed'. ${ }^{14}$

\subsection{Results: Sources of Spatial Inequality}

\subsubsection{Model M0: The null model}

For each year for which we estimate our model, we begin by a four level null model where we introduce nothing but a random intercept on the community, the provincial and the regional level. Using a likelihood ratio test we check whether the three level model, nested in the four level model, performs better than the four level model (see Goldstein (2003)). Since this is not the case for any of the three years under consideration, we will use a four level model in the following.

Our base model, M0, reads:

$$
Y_{i j k l}=\gamma_{0000}+W_{l}+V_{k l}+U_{j k l}+\varepsilon_{i j k l},
$$

where $Y_{i j k l}$ stands for log of household expenditure per capita. The results of model M0 for each year are shown in tables $2.3-2.5$.

\footnotetext{
${ }^{14}$ The estimation procedure is based on an iterative generalized least squares approach (discussed in Goldstein (2003)). This procedure starts with the estimation of the fixed effects coefficients using ordinary least squares. The resulting residuals are stored. Afterwards, an iterative procedure begins, starting with a generalized least squares regression in a first step. Then, in a second step the residuals of this regression are used to compute the variance of the random coefficients. These steps are then iterated.
} 
Table 2.3: Models - 1994 - Fixed effects

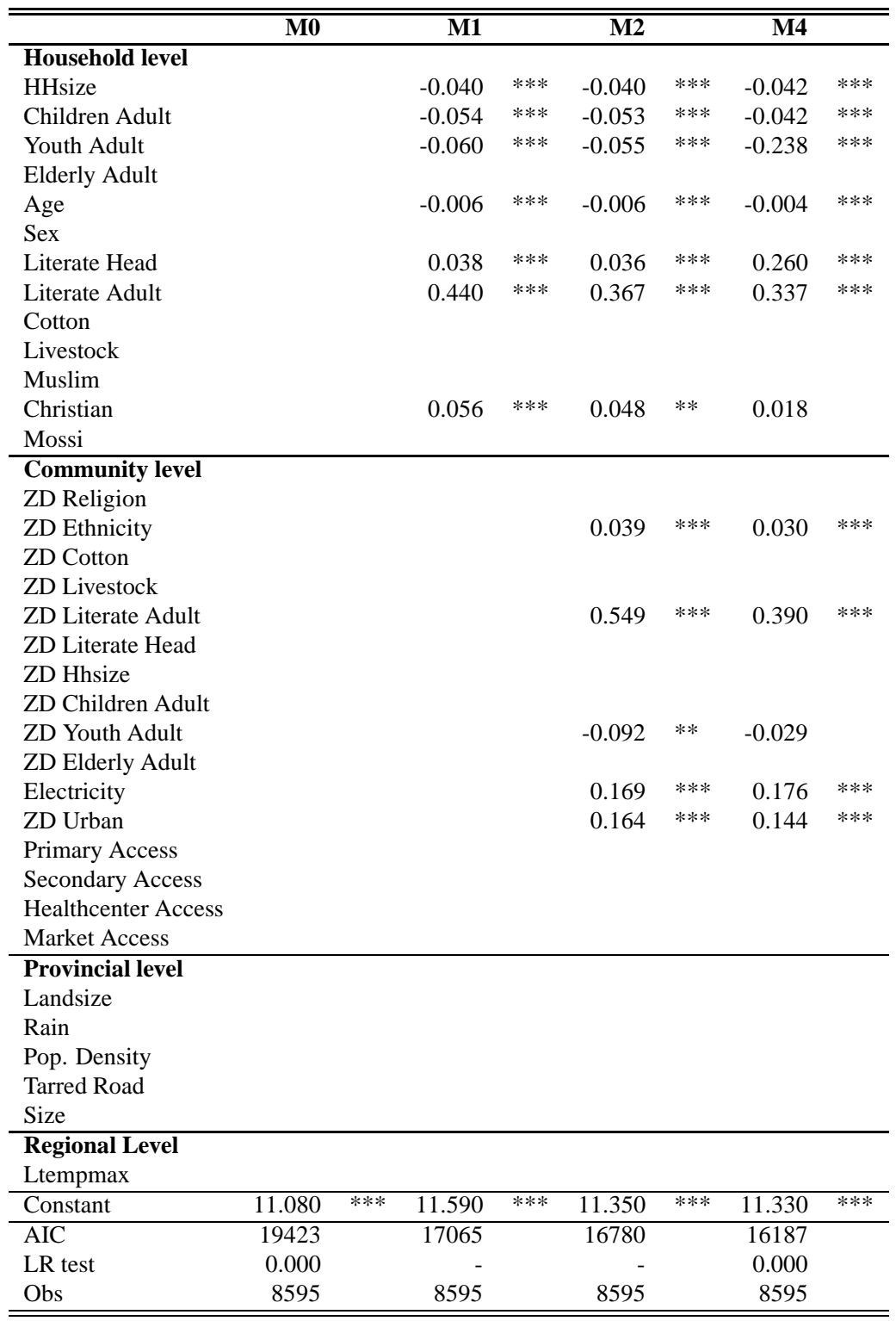

Source: Authors' calculations based on individual dataset 


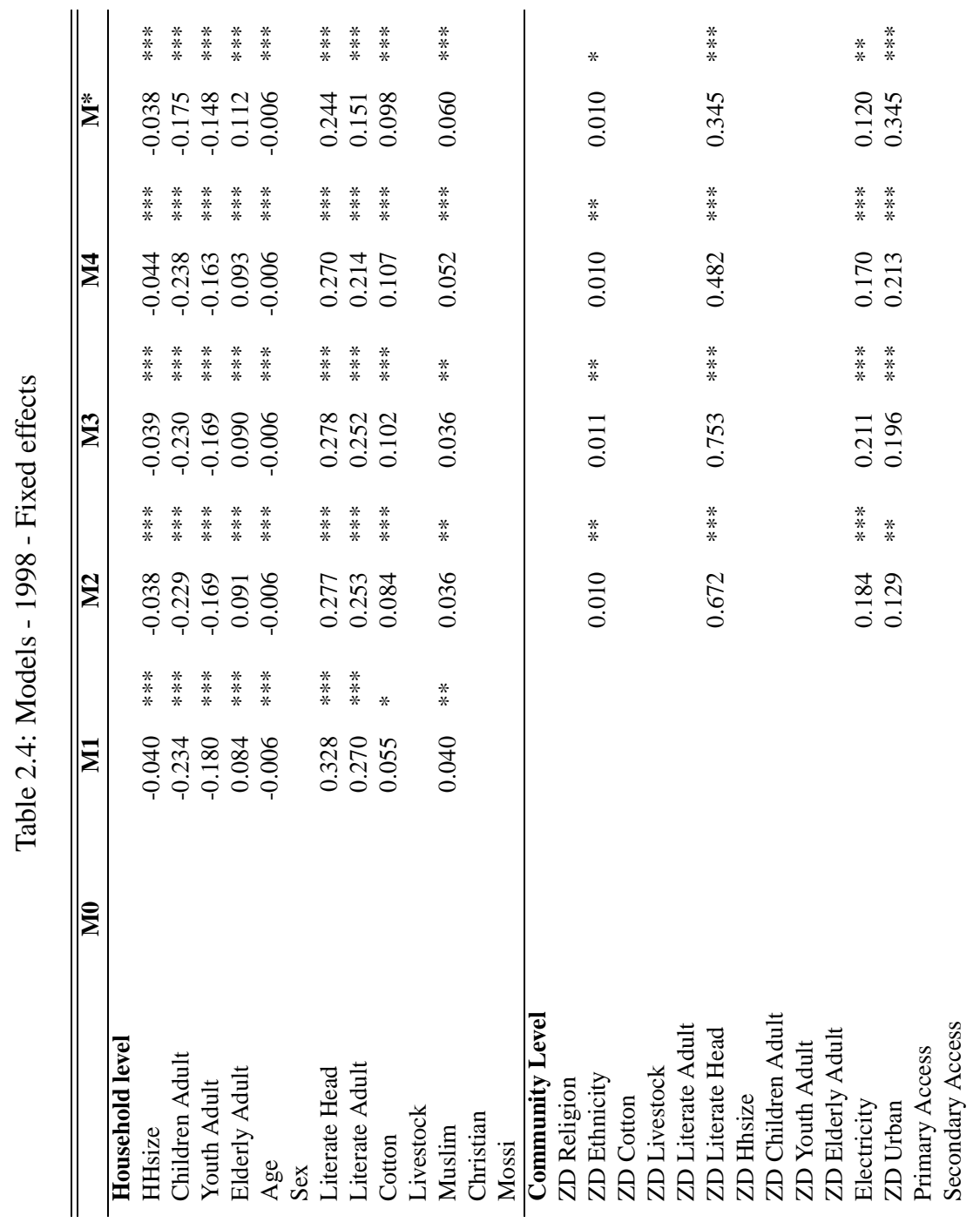




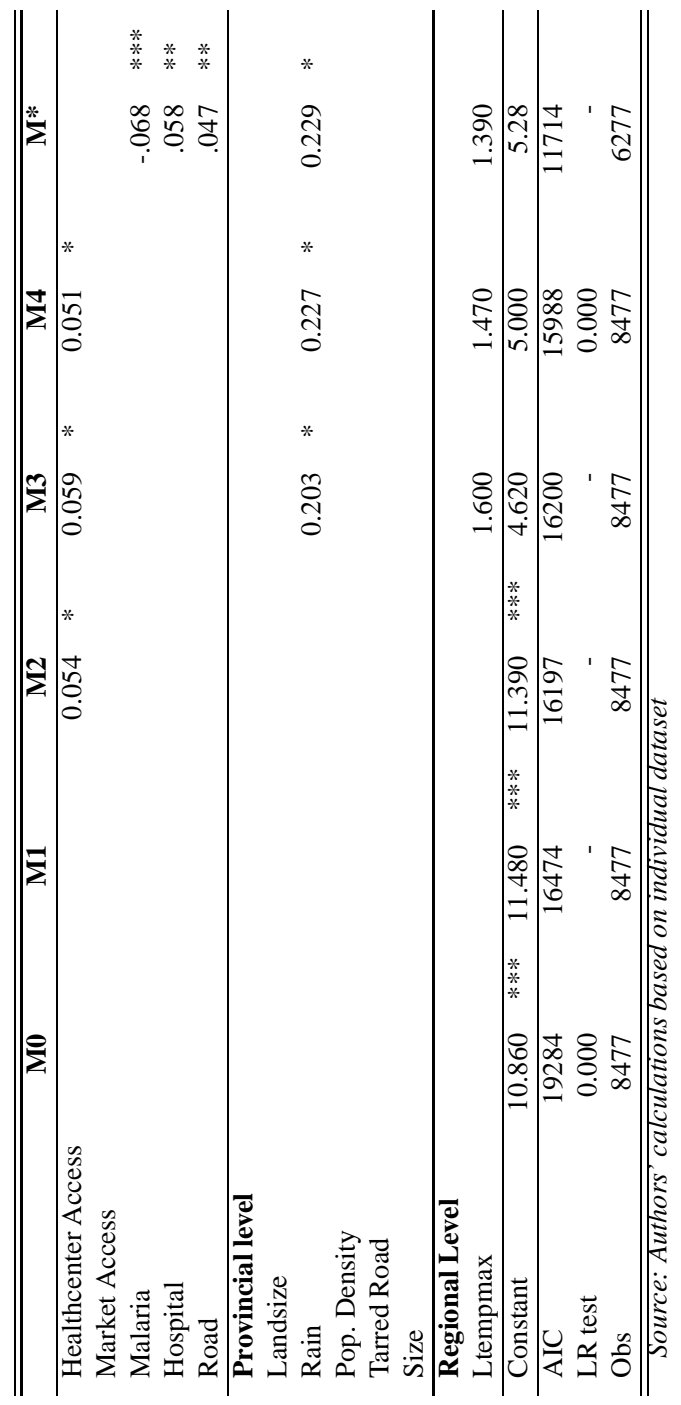

Johannes Gräb - 978-3-653-00480-9 
To obtain the contribution of the variance at each level to the total variance, we calculate the icc for each level. Recall that the icc (e.g. for level 2) is written as:

$$
\rho=\frac{\sigma_{u}^{2}}{\sigma_{u}^{2}+\sigma_{v}^{2}+\sigma_{w}^{2}+\sigma_{\varepsilon}^{2}}
$$

The icc for all years and levels are shown in Table 2.6. For instance, the intra-class correlation coefficient, $\rho$, for the community level in the year 1998, is equal to approximately $26.5 \% .^{15}$ In words, in 199826.5 percent of the total variance is situated at the community level. In this case, the icc measures the correlation of the residual of the response variable of households stemming from the same community. The high icc of the community level, which is almost as high in 1994 (19.2 percent) and 2003 (20.5 percent), depicts two things. First, it underlines the importance of using a multilevel approach to get efficient estimates. Second, it suggests strong community effects which are relatively stable over time. The latter finding is particularly interesting in our case, since it means that the more households' incomes within a community are alike, the more likely is it that incomes are directly related to the contextual environment of the communities (see Merlo et al. (2005a)).

Clearly, most of the variance exists at the household level. It should be emphasized, however, that household expenditure data in developing countries is usually measured with error, given that it is generally very difficult to get precise information on expenditures if simple recall questions are used. Our model attributes the total variance which is due to measurement error in the expenditure data to the household level component. If we were able to account for these errors, the contribution of the household level variance to the total variance would probably be lower, and, in consequence, the contribution of the higher levels higher. The contribution of the variance on the provincial and regional level is relatively low. We conclude - at this stage - that differences in household incomes are mainly driven by household and community (or cluster) characteristics and to a smaller extent by regional characteristics. The contribution of the provincial level is very low. In fact, in Burkina Faso regions rather than provinces follow agro-climatic zones, this can explain why regions make a higher contribution than provinces.

As explained above the finding of a significant contribution of higher level characteristics on income does not necessarily have to be the result of differences in higher level characteristics itself. For instance, differences between communities might result from a systematic distribution of household characteristics across communities, i.e. similar households are spatially concentrated. To see whether this is the case, we have to test the proportional change in the variance components, the random intercepts, after accounting for household characteristics, i.e.

\footnotetext{
${ }^{15} \rho=\frac{.221}{.221+.025+.078+.510} \approx 26.5$ percent.
} 
Table 2.5: Models - 2003 - Fixed effects

\begin{tabular}{|c|c|c|c|c|c|c|c|c|}
\hline \multirow{2}{*}{ Household level } & \multicolumn{2}{|l|}{ M0 } & \multicolumn{2}{|l|}{ 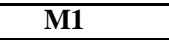 } & \multicolumn{2}{|l|}{ M2 } & \multicolumn{2}{|l|}{$\overline{\mathrm{M4}}$} \\
\hline & & & & & & & & \\
\hline HHsize & & & -0.054 & $* * *$ & -0.054 & $* * *$ & -0.060 & $* * *$ \\
\hline Children Adult & & & -0.227 & $* * *$ & -0.219 & $* * *$ & -0.206 & $* * *$ \\
\hline Youth Adult & & & -0.190 & $* * *$ & -0.178 & $* * *$ & -0.183 & $* * *$ \\
\hline Elderly Adult & & & & & & & & \\
\hline Age & & & -0.004 & $* *$ & -0.004 & $* *$ & -0.004 & $* *$ \\
\hline Sex & & & -0.060 & & -0.064 & & -0.074 & \\
\hline Literate Head & & & 0.272 & $* * *$ & 0.232 & $* * *$ & 0.252 & $* * *$ \\
\hline Literate Adult & & & 0.268 & $* * *$ & 0.2538 & $* * *$ & 0.212 & $* * *$ \\
\hline Cotton & & & 0.079 & $* * *$ & 0.117 & $* * *$ & 0.105 & $* * *$ \\
\hline Livestock & & & 0.034 & $* * *$ & 0.083 & $* * *$ & 0.096 & $* * *$ \\
\hline Muslim & & & & & & & & \\
\hline Christian & & & & & & & & \\
\hline Mossi & & & & & & & & \\
\hline Community level & & & & & & & & \\
\hline ZD Religion & & & & & & & & \\
\hline ZD Ethnicity & & & & & & & & \\
\hline ZD Cotton & & & & & & & & \\
\hline ZD Livestock & & & & & -0.347 & $* * *$ & -0.370 & $* * *$ \\
\hline ZD Literate Adult & & & & & -0.260 & $*$ & -0.290 & $* *$ \\
\hline ZD Literate Head & & & & & 0.827 & $* * *$ & 0.706 & $* * *$ \\
\hline ZD Hhsize & & & & & & & & \\
\hline ZD Children Adult & & & & & & & & \\
\hline ZD Youth Adult & & & & & & & & \\
\hline ZD Elderly Adult & & & & & & & & \\
\hline Electricity & & & & & 0.138 & $* * *$ & 0.146 & $* * *$ \\
\hline ZD Urban & & & & & & & & \\
\hline Primary Access & & & & & & & & \\
\hline Secondary Access & & & & & & & & \\
\hline Healthcenter Access & & & & & & & & \\
\hline Market Access & & & & & 0.088 & $* *$ & 0.075 & $*$ \\
\hline Provincial level & & & & & & & & \\
\hline Landsize & & & & & & & & \\
\hline Rain & & & & & & & & \\
\hline Pop. Density & & & & & & & & \\
\hline Tarred Road & & & & & & & & \\
\hline Size & & & & & & & & \\
\hline Regional Level & & & & & & & & \\
\hline Ltempmax & & & & & & & & \\
\hline Constant & 11.150 & $* * *$ & 11.780 & **** & 12.080 & *** & 11.870 & $* * *$ \\
\hline AIC & 19143 & & 16305 & & 16132 & & 15976 & \\
\hline LR test & 0.000 & & - & & - & & 0.000 & \\
\hline Obs & 8488 & & 8488 & & 8488 & & 8488 & \\
\hline
\end{tabular}

Source: Authors' calculations based on individual dataset 
Table 2.6: Intraclass correlation coefficients (ICC)

\begin{tabular}{|c|c|c|c|c|c|c|c|c|c|c|}
\hline & \multicolumn{3}{|c|}{1994} & \multicolumn{4}{|c|}{1998} & \multicolumn{3}{|c|}{2003} \\
\hline & M0 & M1 & M2 & Mo & M1 & M2 & M3 & Mo & M1 & M2 \\
\hline Region & $9.6 \%$ & $7.8 \%$ & $3.5 \%$ & $9.3 \%$ & $5.0 \%$ & $1.2 \%$ & $1.4 \%$ & $11.1 \%$ & $6.2 \%$ & $3.9 \%$ \\
\hline Province & $4.1 \%$ & $2.1 \%$ & $1.0 \%$ & $3.0 \%$ & $3.1 \%$ & $2.1 \%$ & $2.0 \%$ & $3.3 \%$ & $6.3 \%$ & $7.8 \%$ \\
\hline Community & $21.9 \%$ & $15.9 \%$ & $7.4 \%$ & $26.5 \%$ & $18.9 \%$ & $9.0 \%$ & $9.3 \%$ & $20.5 \%$ & $15.1 \%$ & $9.0 \%$ \\
\hline Households & $64.4 \%$ & $74.2 \%$ & $88.1 \%$ & $61.2 \%$ & $72.9 \%$ & $87.7 \%$ & $87.3 \%$ & $65.2 \%$ & $72.5 \%$ & $79.3 \%$ \\
\hline
\end{tabular}

Source: Authors' calculations based on individual dataset

to control for systematic differences in household characteristics across higher levels. However, it should be noted, that household characteristics might lie in the causal pathway between area characteristics and household income, e.g. better and more schools may lead to better education outcomes. Including household characteristics will probably lead to an understatement of the importance of area characteristics. Hence, it is important to carefully discuss the household level variables and the potential influence of area characteristics on these variables.

\subsubsection{Model M1: The role of household characteristics}

In the second step, we add explanatory variables on the household level to the random intercept model. We call this model 'M1'. The results are presented for each year separately - in Tables $2.3-2.5$. Since we use maximum likelihood techniques for estimation, we rely on the Akaike Information criterion (AIC) to select the best model. We estimated other versions of M1 with a much larger set of potentially important explanatory variables, but present here only those models with the lowest AIC.

\section{Key household level characteristics}

All household variables have the expected sign and are in line with standard regression results. In particular, household composition has a considerable effect on income levels. In terms of per capita incomes, smaller households seem to be significantly better off in all years under consideration. The dependency ratios, measured via the children ( $0-6$ years) per adult ratio, the youth (7-14 years) per adult ratio and the elderly (55 years and older) per adult ratio do all have a significant effect. While young household members lower per capita income in all years, the old-age dependency ratio is insignificant in 1994 and 2003 (thus dropped from the regression for those years) and negative in the drought year 1998 when food prices were extremely high.

Age of the household head has a significant negative effect on household income in all years. The household head being a male adult does not seem to play Johannes Gräb - 978-3-653-00480-9 
a major role concerning income since its effect is only significantly positive in 2003. The education of the household head is, as expected, very important in all years. Households with a literate head and households with a higher percentage of literate adults have on average a higher household income. Ethnicity has no influence on household income. Religion does. Belonging to one of the two large religious groups in Burkina Faso - Islam and Christianity - has a positive, but only barely significant effect on income.

The effect of cotton farming differs across periods. Cotton farmers were better off in 1998 and 2003. In 1994 cotton did not yet have a significant effect. This is plausible, since the 'cotton boom' set in after the devaluation of the CFA Franc in January 1994, enhanced by a very favorable evolution of cotton prices and accompanied by a substantial expansion of land used for cultivation. Farmers who were also engaged in livestock herding which is often done to diversify risk, and hence, to lower the vulnerability to external shocks, were significantly better off in 2003. However, a deeper analysis of this issue would require to take into account the possible endogeneity, since richer farmers are more likely to be engaged in livestock herding than poorer farmers. For the latter, the income constraint does not allow to buy any livestock. Obviously, it is now interesting to see whether for all these household characteristics the effects differ across communities.

\section{Contribution of household characteristics to spatial variation}

For all years the community and regional variance components decline after the incorporation of household level covariates. For the provincial component the direction of the change is unstable for the different years which is not surprising given the small size and low significance of the provincial random intercept. The proportional changes of the community and regional variance components are surprisingly stable across survey years (see Table 2.7). Controlling for household level characteristics reduces the community variance component by around 50 percent.

Table 2.7: Proportional change of variance components

\begin{tabular}{lccccccc}
\hline \hline & \multicolumn{2}{c}{1994} & \multicolumn{3}{c}{$\mathbf{1 9 9 8}$} & \multicolumn{2}{c}{$\mathbf{2 0 0 3}$} \\
& M1 & M2 & M1 & M2 & M3 & M1 & M2 \\
\hline \multirow{2}{*}{ Region } & $-42.5 \%$ & $-62.1 \%$ & $-65.4 \%$ & $-79.6 \%$ & $15.5 \%$ & $-62.4 \%$ & $-42.3 \%$ \\
Province & $-64.0 \%$ & $-60.4 \%$ & $-33.7 \%$ & $-45.0 \%$ & $-3.2 \%$ & $29.0 \%$ & $13.2 \%$ \\
Community & $-49.1 \%$ & $-61.1 \%$ & $-54.6 \%$ & $-60.5 \%$ & $3.4 \%$ & $-50.3 \%$ & $-45.8 \%$ \\
Households & $-19.1 \%$ & $-0.2 \%$ & $-23.9 \%$ & $-0.2 \%$ & $0.0 \%$ & $-25.1 \%$ & $-0.2 \%$ \\
\hline \hline
\end{tabular}

Source: Authors' calculations based on individual dataset 
Abstracting from unobserved household characteristics we would conclude that 50 percent of the community level variation in income levels is due to a systematic distribution of household characteristics across communities while the rest is due to community characteristics. Clearly, this is an unrealistic assumption and would lead to an overestimation of the importance of area-specific effects. Instead we have to consider that household characteristics are itself influenced by higher level factors. Levels as well as returns to education, cotton farming and livestock herding might be influenced by community characteristics, which could be responsible for an underestimation of area importance. Testing the explanatory power of community characteristics itself is therefore essential to draw conclusions on the contribution of community differences on household income disparities.

On the regional level the inclusion of household level variables was also nonambiguous. In 1998 and 2003 observed household characteristics can explain about 60 percent of the total unexplained regional variance (40 percent in 1994). Given that we controlled for household characteristics to the extent possible, we conclude for regions as well that large scale variables have a non-negligible impact on household level income.

\subsubsection{Model M2: The role of community characteristics}

To test for the meaningfulness of our results which indicate a high importance of community characteristics, we will check the proportional change of the variance components after the incorporation of community characteristics (Model M2). The remaining significant variation of the community level random intercept could be either due to unobserved household characteristics leaving the community random intercept more or less unchanged or due to community characteristics (observed or not) lowering the community random intercept towards zero. Again, we use the AIC as a model selection criterion and present only the best fits of the M2 model (see Tables 2.3 - 2.5). All community variables which were tested for significance are listed in Table 2.2.

\section{Key community level characteristics}

If the community matters, the question is of course which are the relevant factors. Tables 2.3 - 2.5 reveal a distinct pattern across the three years. Urban communities with a high ethnic fragmentation ${ }^{16}$, a high share of literate household heads and adults, and access to electricity are better off, on average. Besides the direct effect

\footnotetext{
${ }^{16}$ Ethnic fragmentation is measured as the variance of the shares of each ethnicity in a community.
} 
of having a literate adult in the household, there seems to exist a contextual or spill-over effect of better educated on less educated individuals within communities. However, access to primary and secondary schools - as measured by the time needed to reach them - does not turn out to be significant. Education is the only household characteristic which appears to have some spill-over effects. Except for youth per adult in 1994, all community averages of household level characteristics turn out to be insignificant. This is also true for communities with a higher share of cotton farmers, even though cotton farmers themselves are better off in 1998 and 2003, and cotton is always found to be a factor with some contextual effect in a community.

Since we do not have a direct measure of electricity in a community, we coded a community to have access to electricity if at least one household in that community had access. Electricity might be a good proxy for infrastructure, such as access to roads, in a community, since power transmission lines are usually found along (gravel) roads. Since at the community level we only have information on electricity but not on other infrastructure such as roads, we interpret the positive effect of electricity carefully as a general positive effect of community infrastructure on household income. Though, access to schools, access to health-centers and access to markets turns out to be insignificant. The effect of these kind of public services might be, at least to some extent, captured by the significant positive effect of urban communities since all these services are usually provided in urban areas.

As mentioned in Section 3, in 1998, the household survey was accompanied by a community survey for 325 out of the 425 clusters. This much larger community level dataset in 1998 can however only be examined at the cost of loosing a fourth of all households in the sample. Hence, we report regression results using data for the community survey separately in model $\mathrm{M}^{*}$ in Table 2.4. Of all community survey variables listed in Table 2.2 only access to a road and to a hospital and a high malaria incidence in a cluster affect significantly household income. Signs are as expected. These results confirm the findings derived from model M2. Beyond the positive effect of urbanicity, access to markets and schools do not seem to play a major role in determining household income. Access to roads however - as already suggested by the positive effect of electricity in model M2, which we thought to be highly correlated with road access - seems crucial in raising the potential for income generation.

\section{Contribution of community characteristics to spatial variation}

After accounting for community factors, the community variance component reduces significantly in all years (see Table 2.7). Around 60 percent of the remaining unexplained community level variation in M1 could be explained by observed 
community factors in 1994 and 1998. In 2003 it was still more than 40 percent. Although we only have a modest database on community level variables, this small set of variables is capable of explaining a significant part of the observed between community differences. Hence, in addition to simply specifying some significant relationship between contextual variables and household income as done above, we conclude that these variables are actually responsible for a large part of the community level disparity. ${ }^{17}$ Community endowments have a significant effect on household income.

The variance partitioning does even allow to quantify its contribution to total income variation. The very limited set of neighborhood characteristics contributes to approximately $7 \%^{18}$ of household income variation. Since we are neglecting any measurement error as well as any effects from the community on household characteristics this result can be seen as a lower bound of the contribution of community characteristics to total income variation.

However, the question remains whether provincial and regional income disparities, that were persistent after controlling for household characteristics, are actually driven by differences in provincial and regional endowments or whether they are mainly driven by differences in community characteristics between these areas. Table 2.7 shows that around 60 percent of the remaining regional level variation in 1994, 80 percent in 1998 and 40 percent in 2003, can be explained by differences in observed community endowments. After the consideration of household and community level determinants, less than 5 percent in 1994 and 1998 and less than 12 percent in 2003 of the remaining total unexplained variation is situated at the provincial and regional level together. Here again, it should be noted that lower level factors are likely to be driven by macro factors, and, hence, we risk to understate the influence of variables on higher aggregation levels. Moreover, likelihood ratio tests show that both levels still have a significant impact.

\subsubsection{Model M3: The role of provincial and regional charac- teristics}

In model M3 we incorporate provincial and regional level variables. However, except for the 1998 rainfall variable (the drought year), all provincial and regional variables turned out to be insignificant. Population density, the density of tarred and gravel roads, the average maximum temperature or the variation of rainfall did

\footnotetext{
${ }^{17}$ The remaining unexplained community variation cannot not be dissolved with our data at hand.

${ }^{18}$ For instance for the year 1998: ICC(M0)*(1-proportional change of variance component(M1))*proportional change of variance component(M2)=.265*(1-.546)*.605 $=7.3$ percent.
} 
not show a significant effect, once household and community level characteristics were included. The remaining unexplained variation could not be lowered in any of the three years under consideration. Table 2.8 summarizes the contribution of observed and unobserved characteristics to the total variance and the variance on each spatial level.

Table 2.8: Contribution of observed and unobserved characteristics on the variation on each level

\begin{tabular}{lccc}
\hline \hline & $\mathbf{1 9 9 4}$ & $\mathbf{1 9 9 8}$ & $\mathbf{2 0 0 3}$ \\
\hline Household level & $\mathbf{6 4 . 4 \%}$ & $\mathbf{6 1 . 2 \%}$ & $\mathbf{6 5 . 2 \%}$ \\
Household variables & $19.1 \%$ & $23.9 \%$ & $25.1 \%$ \\
Unobserved & $80.9 \%$ & $76.1 \%$ & $74.9 \%$ \\
\hline & & & \\
Community level & $\mathbf{2 1 . 9 \%}$ & $\mathbf{2 6 . 5 \%}$ & $\mathbf{2 0 . 5 \%}$ \\
Household variables & $49.1 \%$ & $54.6 \%$ & $50.3 \%$ \\
Community variables & $31.1 \%$ & $27.5 \%$ & $22.7 \%$ \\
Unobserved & $19.8 \%$ & $17.9 \%$ & $27.0 \%$ \\
\hline & & & \\
Provincial level & $\mathbf{4 . 1 \%}$ & $\mathbf{3 . 0 \%}$ & $\mathbf{3 . 3 \%}$ \\
Household variables & $64.0 \%$ & $33.7 \%$ & $<10^{-3}$ \\
Community variables & $21.7 \%$ & $29.8 \%$ & $<10^{-3}$ \\
Provincial/Regional variables & $<10^{-3}$ & $1.2 \%$ & $<10^{-3}$ \\
Unobserved & $14.3 \%$ & $35.3 \%$ & $>99 \%$ \\
\hline & \multicolumn{3}{|c}{} \\
Regional level & $\mathbf{9 . 6 \%}$ & $\mathbf{9 . 3 \%}$ & $\mathbf{1 1 . 1 \%}$ \\
Household variables & $42.5 \%$ & $65.4 \%$ & $62.4 \%$ \\
Community variables & $35.7 \%$ & $27.5 \%$ & $15.9 \%$ \\
Provincial/Regional variables & $<10^{-3}$ & $<10 \%$ & $<10^{-3}$ \\
Unobserved & $21.8 \%$ & $7.1 \%$ & $21.7 \%$ \\
\hline \hline
\end{tabular}

Source: Authors' calculations based on individual dataset

The result of insignificant macro-level variables might seem surprising, but it is in fact quite consistent with other findings in the literature. Jalan and Ravallion (2002) and Benson et al. (2005) do also not find a significant effect of population density on household income. Benson et al. (2005) even confirm our result of a missing effect of access to roads which is according to Jacoby (2000) the result of a low infrastructure elasticity of poverty.

Burkinabe households seem to have adapted their income generation process to the inherent climatical disadvantages in a way that the amount and the variation of rainfall in 'normal times' does not have a significant impact on their income. However, the occurrence of substantial climatic shocks, such as a drought or an abnormal distribution of rainfall over the year, do play an important role, as revealed by the significant positive rainfall coefficient in the drought year 1998. 
Consistent with this result, Benson et al. (2005) find the effect of the amount of rainfall on income in Malawi only to be significant when it is exceptionally high. Similarly, Dercon (2004) only finds significant effects for Ethiopia when looking at severe droughts.

Our results are also in line with those by Bigman et al. (2000) who conclude that regional inequality in Burkina Faso is driven by agro-climatic conditions, and disparities between villages are driven by differences in infrastructure. However, compared to Bigman et al. (2000), we stress the importance of community characteristics even more. Our analysis suggests that a large part of regional disparity is actually driven by differences in community characteristics between these regions. Hence, we think the actual impact of agro-climatic conditions is lower than suggested by Bigman et al. (2000).

\subsubsection{Model M4: Variations in household level effects across communities}

In a next step, we allow household level variables to differ in their impact across communities. Thus, in addition to random intercepts, we now also add random coefficients (see equation 2.7) at the community level. Covariances of random effects are modeled unstructured, i.e. all variances-covariances are distinctly estimated. We use an iterative procedure to test for significant variance-covariances of all significant household level variables included in model M2. We use likelihoodratio tests by estimating the likelihood deviance for the model without the specific random effect and for the model with the specific random effect. We keep those random effects in model M4 whenever the test-statistic - the difference between the deviances of the two models - is significant, i.e. if we get a $\chi^{2}$ below $5 \%$ (Goldstein, 2003). In addition, variances and covariances are regarded as insignificant when their standard error is larger than their estimate (Tseloni, 2006). All estimates and their standard errors for model M4 are shown in Tables $2.9-2.10$. 
Table 2.9: Models - 1994 - Random effects

\begin{tabular}{|c|c|c|c|c|c|c|c|c|}
\hline & \multicolumn{2}{|c|}{ M0 } & \multicolumn{2}{|c|}{ M1 } & \multicolumn{2}{|c|}{ M2 } & \multicolumn{2}{|c|}{$\overline{\mathrm{M4}}$} \\
\hline & Est. & Std.Err. & Est. & Std.Err. & Est. & Std.Err. & Est. & Std.Err. \\
\hline Variances & & & & & & & & \\
\hline var(region) & 0.075 & 0.047 & 0.043 & 0.023 & 0.016 & 0.008 & 0.015 & 0.008 \\
\hline var(province) & 0.032 & 0.018 & 0.012 & 0.007 & 0.005 & 0.003 & 0.006 & 0.003 \\
\hline var(community) & 0.171 & 0.014 & 0.087 & 0.008 & 0.034 & 0.004 & 0.091 & 0.012 \\
\hline var (household) & 0.502 & 0.008 & 0.406 & 0.006 & 0.406 & 0.006 & 0.360 & 0.006 \\
\hline $\operatorname{var}($ hhsize $)$ & & & & & & & 0.000 & 0.000 \\
\hline $\operatorname{var}($ youth adult) & & & & & & & 0.018 & 0.006 \\
\hline var(liter. head) & & & & & & & 0.055 & 0.013 \\
\hline Covariances & & & & & & & & \\
\hline $\begin{array}{l}\text { cov(hhsize, } \\
\text { youth adult) }\end{array}$ & & & & & & & 0.000 & 0.001 \\
\hline $\begin{array}{l}\text { cov(hhsize, } \\
\text { lit. head) }\end{array}$ & & & & & & & -0.002 & 0.001 \\
\hline $\begin{array}{l}\operatorname{cov}(\text { youth ad, } \\
\text { lit. head) }\end{array}$ & & & & & & & -0.007 & 0.007 \\
\hline $\operatorname{cov}($ hhsize,cons) & & & & & & & -0.005 & 0.001 \\
\hline $\begin{array}{l}\text { cov(youth ad, } \\
\text { cons) }\end{array}$ & & & & & & & -0.018 & 0.007 \\
\hline $\begin{array}{l}\text { cov(lit. head, } \\
\text { cons) }\end{array}$ & & & & & & & 0.009 & 0.009 \\
\hline
\end{tabular}

Table 2.10: Models - 2003 - Random effects

\begin{tabular}{|c|c|c|c|c|c|c|c|c|}
\hline & \multicolumn{2}{|c|}{ M0 } & \multicolumn{2}{|c|}{ M1 } & \multicolumn{2}{|c|}{ M2 } & \multicolumn{2}{|c|}{ M4 } \\
\hline & Est. & Std.Err. & Est. & Std.Err. & Est. & Std.Err. & Est. & Std.Err. \\
\hline Variances & & & & & & & & \\
\hline $\operatorname{var(region)}$ & 0.085 & 0.045 & 0.032 & 0.021 & 0.019 & 0.013 & 0.019 & 0.0137 \\
\hline $\operatorname{var}$ (province) & 0.025 & 0.013 & 0.032 & 0.012 & 0.037 & 0.011 & 0.041 & 0.0122 \\
\hline var(community) & 0.157 & 0.013 & 0.078 & 0.007 & 0.042 & 0.005 & 0.066 & 0.0114 \\
\hline var (household) & 0.502 & 0.008 & 0.376 & 0.006 & 0.375 & 0.006 & 0.350 & 0.0059 \\
\hline var(hhsize) & & & & & & & 0.001 & $\overline{0.000}$ \\
\hline var(youth adult) & & & & & & & 0.005 & 0.004 \\
\hline var(liter. head) & & & & & & & 0.074 & 0.014 \\
\hline Covariances & & & & & & & & \\
\hline $\begin{array}{l}\text { cov(hhsize, } \\
\text { youth adult) }\end{array}$ & & & & & & & 0.002 & 0.0007 \\
\hline $\begin{array}{l}\text { cov(hhsize, } \\
\text { lit. head) }\end{array}$ & & & & & & & -0.003 & 0.0011 \\
\hline $\begin{array}{l}\text { cov(youth ad, } \\
\text { lit. head) }\end{array}$ & & & & & & & -0.012 & 0.0069 \\
\hline $\operatorname{cov}($ hhsize,cons) & & & & & & & -0.004 & 0.0011 \\
\hline $\begin{array}{l}\text { cov(youth ad, } \\
\text { cons) }\end{array}$ & & & & & & & -0.013 & 0.0061 \\
\hline $\begin{array}{l}\text { cov(liter. head, } \\
\text { cons) }\end{array}$ & & & & & & & 0.016 & 0.0094 \\
\hline
\end{tabular}

Source: Authors' calculations based on individual dataset 


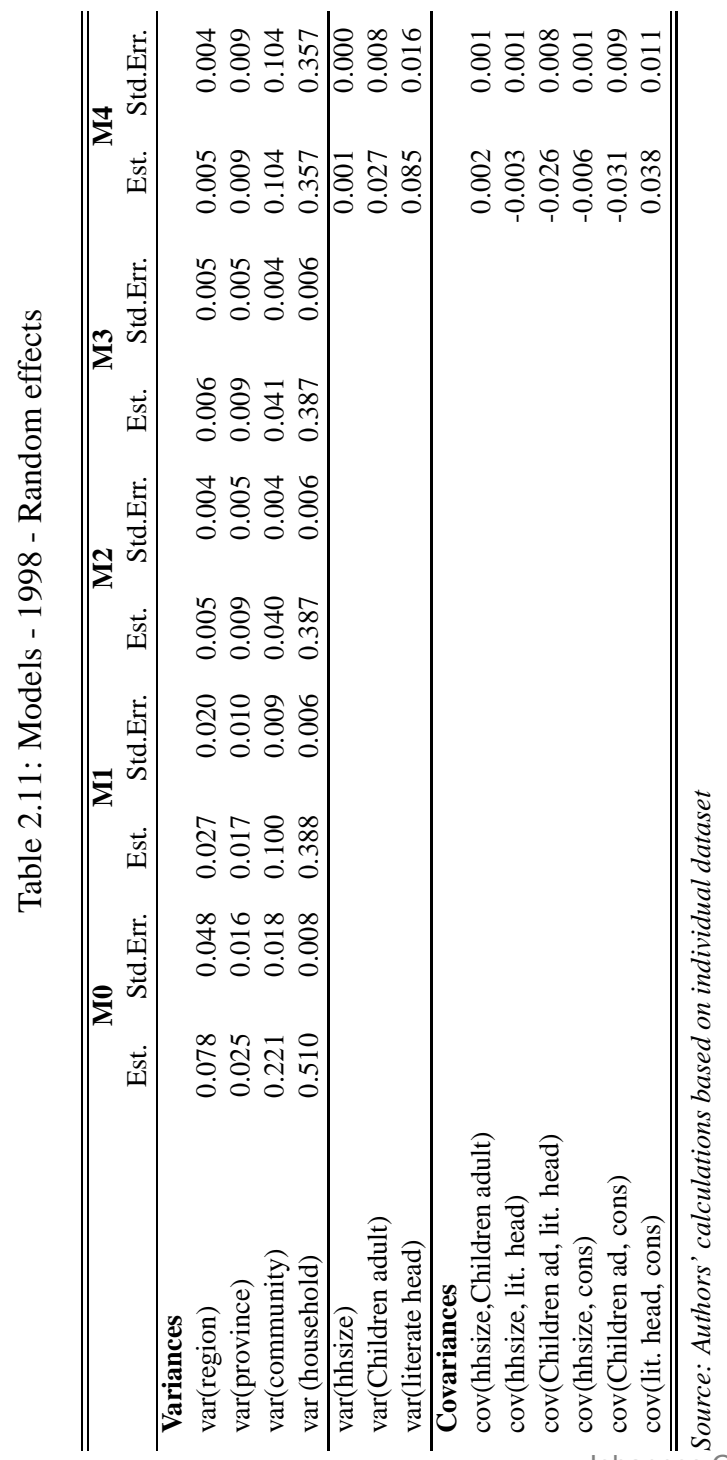




\section{Spatially varying household effects}

The results of our analysis are, once again, relatively homogeneous across time. We find indeed, that returns associated with education, household size and effects related to dependency ratios (children per adult and youth per adult) vary significantly across communities in all three years. On the other hand, returns associated with age and gender of the household head, with cotton farming and livestock herding do not vary significantly across communities in either year.

The variation of returns across communities is not only statistically but also economically meaningful. The fixed effect estimate of the variable 'literate head' of .26 in 1994 states that households with a literate head have on average a per capita income which is higher by 26 percent compared to households with an illiterate head. The variance of the random effect of the household head variable states however that this return differs significantly between communities. For instance for 1994, the effect varies from minus 21 percent $(((.26-2 * \sqrt{.055}) *$ $100))$ to plus 73 percent $(((.26+2 * \sqrt{.055}) * 100))$ between the 2.5 th and 97.5 th quantile of Burkinabe communities. Similar variations are stated for 1998 and 2003. The effects associated with changes in the household composition vary also substantially across communities.

\section{Determinants of spatially varying effects}

We conclude that the community has an influence on effects associated with household characteristics, in particular with education. From a policy point of view, it is important to know what drives these community effects. In the case of returns to education, it might be channeled through unobserved factors like labor market characteristics or the access to modern (agricultural) production technologies. These factors will rather be found in better developed communities. However, higher returns to education could also be the result - decreasing marginal returns to education assumed - of higher marginal effects in some poor and remote communities. While the former case would rather lead to income divergence across communities, the latter could lead to income convergence.

To get further insights we can calculate the best linear unbiased predictors (BLUP) of the random effects and check if variations in returns across communities follow a distinct pattern across the 13 agro-climatic regions in Burkina Faso. ${ }^{19}$ We cannot, however, find any evidence for a North-South or East-West pattern in returns to education across the 13 regions in any year. The same is true for the

\footnotetext{
${ }^{19}$ Since the regression coefficients associated with the household characteristics - which are random variables at the community level - are directly determined by the observed community level factors (see equation 2.3) further regression analysis is not feasible. 
household size and dependency ratios. ${ }^{20}$ We conclude that returns to these factors are driven by small scale community characteristics but not by any regional factor.

We can also examine the covariance of random effects and random intercepts. For the returns to education the covariance between its random effect and the random community intercept turns out to be insignificant in 1994 and positively significant in 1998 and 2003. On average returns to education are higher in richer communities, ceteris paribus. Again, this may point to the impact of unobserved community factors on educational returns. As stated above, labor markets are usually better developed in richer communities in a sense that they are offering more opportunities for a better educated and trained work force. Moreover, modern agricultural inputs which may require skilled labor are rather found in richer communities. Hence, there is little evidence for higher returns to education in poorer communities. This is probably due to an only weakly competitive labor market and the general low demand for skilled labor in rural areas of poor countries such as Burkina Faso. Therefore, we conclude that disparities in returns to education cannot explain convergence across districts.

Regarding the effect of household size, the covariance with the community intercept is significantly negative in all years. The same is true for the effects associated with dependency ratios; children and youth per adult. This is an interesting result, stating that an additional household member, at working age or not, lowers per capita income more in richer than in poorer communities. In the Burkinabe context, it might just show that it is easier for an agricultural than for an urban household to feed and sustain an additional household member. ${ }^{21}$

\subsection{Conclusion}

The objective of this paper was to analyze the sources of spatial disparities in income among households in Burkina Faso. We find that about 60 percent of the total variance in incomes stems from variance between households, 20 percent from the variance between communities, less than 5 percent from the variance between provinces and about 10 percent from the variance between (agro-climatic) regions. Within each level community characteristics play a very important role. In particular our findings suggest that communities and provinces are not only poor because the households which live there are poor but also because the endowments of these communities are very weak (and vice versa for rich communities). Differences in observed community characteristics account also for a large part of the regional variation. Hence, community characteristics matter.

\footnotetext{
${ }^{20}$ Results can be found in the appendix (Figures A.8-A.16). Codes are defined in Table A.9.

${ }^{21}$ Our finding of a negative covariance between intercepts and household size and dependency ratios do also hold when Ouagadougou is dropped from the regression. 
We also find that the effects associated with household's education and their size and composition are community-specific. For instance, we find higher returns to education in the rather richer communities. In contrast, returns to cotton farming and livestock herding are more or less constant across these spatial units.

One may tend to conclude from our analysis that poverty alleviation policies should intervene at the community level, since at that level we identify the most important source of variance, and hence interventions at the regional or national level would risk to waste resources. However, political and institutional constraints might make it difficult to intervene at that level. This has to be studied case by case.

Finally, it should be noted that our analysis is constrained by the limited availability and the modest quality of data at the different spatial levels. In Burkina Faso, as well as in many other developing countries, community surveys are missing. Geo-referenced data is also often not available. However, as we show, smallscale area data is key to understand and tackle spatial disparities in income. 


\title{
Essay 3
}

\section{Low Malnutrition but High Mortality: Explaining the Paradox of the Lake Victoria Region}

\begin{abstract}
Exploiting DHS data from 235 regions in 29 Sub-Saharan Africa countries, we find that the combination of low levels of malnutrition together with dramatically high rates of mortality, encountered in Kenya's Lake Victoria territory, is unique for Sub-Saharan Africa. This paper explores the causes of this paradox for the Kenyan context. Our identification strategy consists of two parts. First of all, we apply multilevel regression models to control simultaneously for family and community clustering of the observed malnutrition and mortality outcomes. Secondly, to address unobserved but correlated factors, we exploit information from GIS and malaria databases to construct variables that capture additional components of children's geographic, political and cultural environment. Our analysis reveals that beneficial agricultural conditions and feeding practices lead to the observed sound anthropometric outcomes around Lake Victoria. In contrast, high mortality rates rest upon an adverse disease environment (malaria prevalence, water pollution, HIV rates) and a policy neglect (underprovision of health care services). Nonetheless, a significant effect of the local ethnic group, the Luo, on mortality remains.
\end{abstract}

based on joint work with Jan Priebe. 


\subsection{Introduction}

There are a small number of regions in Sub-Saharan Africa that show very high levels of mortality given the anthropometric status of their population. Among these regions Nyanza, the principal Kenyan Lake Victoria province, is an exception of its own. In no other region in Sub-Saharan Africa (SSA) is the pattern of low levels of malnutrition together with dramatically high rates of mortality as pronounced as in Nyanza. Furthermore, the unique position of Nyanza is not only puzzling for Sub-Saharan Africa, but as well in the Kenya specific context. While Nyanza ranges on the upper limit of the mortality scale, most other Kenyan provinces depict comparatively low mortality rates given their levels of malnutrition.

In this paper we investigate the role of cultural, geographic, and political factors on the relationship of anthropometric outcomes of children and under-5 mortality rates in Kenya with an explicit focus on the unique situation of Nyanza and the territory around Lake Victoria. In order to disentangle the underlying mechanism that lead to the observed outcomes we analyze the factors driving mortality, stunting, and wasting jointly. Since parameter estimation can seriously suffer from endogeneity problems we adopted 3 strategies to mitigate this problem. First of all, we estimate reduced form regressions and therefore exclude any explanatory variable that we would expect to cause problems of simultaneous causality. Secondly, we augmented our DHS data by generating appropriate variables on malaria, health provision and Lake Victoria in order to mitigate problems arising from potential omitted variable bias. Thirdly, we use mixed model representations to further address unobserved heterogeneity issues on the family and community level.

Our findings point to a unique interaction of cultural, geographic and political factors in the Lake Victoria region which are responsible for causing the described paradox. Particularly, high mortality rates are found to rest upon the disease environment in the territory in combination with unfavorable cultural habits of the local ethnic group with respect to sexual, and pre- and post natal behavior. Political discrimination against this group resulting in reduced access to health infrastructure further exacerbates the mortality situation in the region. Nonetheless, even after controlling for other factors a significant ethnic specific influence on mortality remains although the effect is much smaller than found in previous studies. On the other hand, the area around Lake Victoria displays extraordinary positive conditions - fertile soils, a high level of food security and high protein availability (fish) - that contribute to children's advantageous nutritional outcomes.

In this regard the existing study adds an important new example and further insights to the few existing mortality-malnutrition paradoxes. For instance, the famous and widely investigated South Asia vs. Sub-Saharan Africa enigma (Rama- 
lingaswami et al., 1996; Svedberg, 2000; Harttgen and Misselhorn, 2006; Klasen, 2008) refers to the observation that anthropometric outcomes of children are on average much better in SSA than in South Asia, while on the other hand child mortality rates are significantly higher in SSA compared to South Asia. ${ }^{1}$ Our study contributes to this literature for a variety of reasons. First of all, we are the first to explicitly state the Lake Victoria paradox and to analyze it comprehensively. Secondly, the study illustrates that not only in the context of potential large genetic differences (Klasen, 2008) such a paradox can arise, but that an interaction of cultural, geographic and political factors can reverse the positive relationship between a good nutritional status and the survival chances of children. Thirdly, in contrast to previous empirical studies on the South Asia vs. SSA enigma we explicitly control for factors related to the disease environment, e.g. HIV and malaria, and cultural factors and therefore are in a better position to obtain unbiased coefficients of our estimates. Fourthly, we use recent advances in multilevel modeling techniques that allow for the estimation of 3-level models which enables us to separate effects working at the individual, household, and community level. Moreover, this study is to our knowledge the most complete and accurate one analyzing the current determinants of under- 5 mortality and anthropometric outcomes in Kenya.

Examining the nature of the Nyanza anomalies is further interesting and important since this example seems to question main findings in a variety of academic disciplines, most notably epidemiology, health economics, labor economics and economic history. In epidemiology, malnutrition, in particular wasting, low weight-for-height, is considered to be the main driver of mortality in developing countries (Villamor et al., 2005; Fawzi et al., 1997) and historical Europe (Fogel, 1994). Pelletier et al. (1995) claim that wasting, which is positively related to mortality due to diarrhea, fever and breathlessness, is the underlying cause of more than 50\% of all child deaths in the world. Moreover, Caulfield et al. (2004) find that a sound nutritional status of children lowers the likelihood to die from malaria while Villamor et al. (2005) show that among HIV infected children the risk of dying in early ages was significantly higher for children being wasted. Thus, children who suffer from malnutrition have a significantly higher risk exposure to mortality due to a lower resistance to illnesses. This individual relationship is usually assumed to hold even on a higher aggregate level. Thus, areas with high prevalence of malnutrition rates are expected to have higher mortality rates. Regarding the Kenyan context we find the exact opposite pattern. While rates

\footnotetext{
${ }^{1}$ In another mortality-malnutrition paradox Williamson (1990) finds that during the British industrial revolution the population in urban areas suffered from much higher mortality rates than the rural population despite their much better anthropometric status.
} 
of wasted children gradually decrease with proximity to Lake Victoria, mortality rates steadily increase reaching its peak in Nyanza.

In health and labor economics the interest of studying the growth process of children has more recently been motivated by the findings that taller populations are economically better-off, more productive, and live longer (Bozzoli et al., 2009; Deaton, 2008). This result can partly be attributed to the prevailing disease environment. If on the one hand the child growth process and therefore adult heights and on the other hand life expectancy are positively correlated with the lack of certain diseases then for instance a simple Beckerian type of quantity-quality tradeoff models can explain higher incomes of taller populations through the human capital formation process. The relationship between adult height and economic well-being might therefore only be valid if adult height is at the same time a good proxy for the mortality environment of a certain area or a country. In the absence of this later condition the relationship might be seriously flawed and this is what we partly observe in the Kenyan context.

Furthermore, there exist several studies in the field of economic history that make inferences about economic conditions during a specific time period based on mean height measures of population or population subgroups. Since height is expected to be an increasing but concave function of income, average height will be negatively correlated with initial income inequality (Steckel, 1995). Hence, income inequality has an effect on the dispersion of heights, so that inequality in height might function as an indicator of income inequality in the absence of data on the latter one, while mean height might serve as an indicator of mean income (Deaton, 2008). Obviously this inference does only hold if there are no other third factors that alter the relationship in an important way. Although this point has been recognized in the relevant literature (Deaton, 2008), it is often neglected or downplayed due to a lack of data that can function as control variables. Again the case of Nyanza and Lake Victoria illustrates that such an inference can simply go wrong.

The paper is structured as follows: Section 3.2 amplifies and describes the extent of the paradox of Nyanza and Lake Victoria in the Kenyan and Sub-Saharan African setting with respect to stunting, wasting and under-5 mortality. Section 3.3 discusses the theoretical model and outlines the identification strategy. Section 3.4 provides a detailed literature review on the most relevant cultural, geographical, and political particularities of the Kenyan context in order to explain the construction and interpretation of additional variables not included in DHS surveys. Section 3.5 provides descriptive statistics on the data sets and variables used in this study and comprises the multivariate analysis. Section 3.6 summarizes and concludes. 


\subsection{The Paradox}

Evidence from the health and demographic literature suggests that there exists a clear positive relationship between a good nutritional status of a child and its chances of survival. From this observation it is usually inferred that this individual relationship holds as well at a higher aggregate level. Hence, regions or countries that perform well in terms of anthropometric indicators should exhibit lower mortality rates and vice versa.

However, the validity of this inference seems to be seriously challenged considering the spatial distribution of malnutrition and mortality in Kenya. Comparing the anthropometric and mortality outcomes of children in the Nyanza province to the other Kenyan provinces two things are salient. First of all, children in Nyanza score very well regarding anthropometric indicators, while infant and child mortality rates are extremely high in the region. Secondly, the mere extent of the within country variation in mortality rates astonishes. The extraordinary situation of Nyanza or more precisely the Lake Victoria region in the Kenyan context has been noted already for a long time. An investigation of 16 villages in 1922 in the area north of Lake Victoria showed an infant mortality rate between 335 and 514 per 1,000 live births with a tendency of declining infant mortality with distance to the lake (Colony and Protectorate of Kenya, 1923). Meanwhile historical data on adult mean height for the same time period suggests that the ethnic group living on the shores of Lake Victoria were the tallest in all over Kenya (Moradi, 2009).

Interestingly, although some authors occasionally have mentioned either the high mortality rates or the favorable anthropometric outcomes on the shores of Lake Victoria, to our knowledge no study exists that combines these two findings. Therefore, the puzzling situation on the shores of Lake Victoria has not yet been stated as such in the literature. Moreover, it has not been clear whether the observed mortality pattern combined with favorable anthropometric outcomes on the shores of Lake Victoria is even unusual for a larger geographical context. Hence, in order to assess the peculiarity of the Kenyan Lake Victoria region in the SubSaharan Africa setting we compiled a data set gathering information on anthropometric (prevalence of stunting and wasting) and mortality indicators (under-5 mortality rate) on regional level for all SSA countries where appropriate Demographic and Health Survey (DHS) data was available. If more than one DHS round was available for a country, we chose to only take the latest round into consideration. Furthermore, since the child growth reference standard which is used to calculate $\mathrm{z}$-scores had changed rather recently, anthropometric statistics in our sample obtained from the official DHS reports would be based on two different standards. For the sake of comparison we recalculated stunting and wasting prevalence rates Johannes Gräb - 978-3-653-00480-9 
using the new WHO child growth reference standard for all regions (WHO, 2006). The final data set comprises 235 regions in 29 SSA countries.

Table 3.1 depicts under-5 mortality, stunting, and wasting rates for all regions with an under-5 mortality rate of 200 per 1,000 live births or more. Of these 36 regions only Tambacounda province in Senegal shows better stunting rates than the Nyanza province while with respect to wasting only Tete and Niassa provinces in Mozambique show lower prevalence rates. Moreover, it is interesting to note that all regions in the table that achieve prevalence rates in one of the anthropometric indicators similar to those in Nyanza, score much worse than Nyanza in the other anthropometric indicator. Therefore, Nyanza seems to be the only region in SSA that scores extremely well in stunting and wasting given the level of mortality.

Even more striking is the result when focusing on the area in close proximity to Lake Victoria. Using the 2003 DHS round for Kenya jointly with the provided GIS data we calculated under-5 mortality rates and the two anthropometric indicators for all children born within a distance of $20 \mathrm{~km}$ from Lake Victoria. In this area under- 5 mortality rates strongly increase by approximately $50 \%$ to 306 per 1,000 live births compared to the Nyanza average while stunting rates even fall to $26.6 \%$ with wasting rates increasing slightly to $3.9 \%$. Furthermore, the extreme position of the Lake Victoria region and Nyanza can be nicely illustrated as in Figure 3.1 which depicts the bivariate relationship between stunting and under-5 mortality for all 235 regions and the Lake region as defined above. Figure 3.1 underscores the unusual high mortality level of Nyanza given its stunting rates and much more important the unique position of the Lake area in the SSA context with an overwhelmingly high under-5 mortality rate given the level of stunting. A similar conclusion can be derived from Figure 3.2 which presents the bivariate relationship between wasting and under- 5 mortality.

Besides highlighting the extraordinary situation of the Lake Victoria region, all 3 figures show further puzzling results. In particular, the within country distribution of under-5 mortality rates is remarkable. While Nyanza is situated far on the upper bound of the under-5 mortality rates given its level of stunting or wasting, several Kenyan provinces find themselves on the opposite side, showing very low mortality rates given its level in the respective anthropometric indicators. This high divergence of mortality levels within one country is highly unusual even for the SSA context. Column 9 in Table 1 presents the coefficient of variation (CV) based on the separate calculation for each country. Out of the whole sample Kenya shows the highest values in the CV among all 29 countries indicating the highest level of dispersion given its level of mortality.

Interestingly, the Lake Victoria provinces of Uganda and Tanzania do not exhibit such an unusual pattern as Nyanza. While anthropometric outcomes for children are slightly worse in these provinces compared to Nyanza, under-5 mortality rates are substantially lower. Since geographical and epidemiological conditions 
Table 3.1: Mortality and undernutrition rates in the SSA context

\begin{tabular}{|c|c|c|c|c|c|c|c|c|}
\hline No. & Country & "Region & Year & Mortal. & Stunt. & Wast. & Range & $\overline{\mathrm{CV}}$ \\
\hline 1 & Senegal & Tambacounda & 2005 & 200 & 28.7 & 11.2 & -126 & 0.31 \\
\hline 2 & Zambia & Western & 2001 & 201 & 49.7 & 3.3 & -118 & 0.22 \\
\hline 3 & Burkina Faso & Sud-Ouest & 2003 & 203 & 47.7 & 25.7 & -166 & 0.22 \\
\hline 4 & Chad & Zone8 & 2004 & 204 & 35.2 & 13.9 & -122 & 0.20 \\
\hline 5 & Cameroon & Nord & 2004 & 205 & 49.6 & 8.4 & -130 & 0.27 \\
\hline 6 & Senegal & Kolda & 2005 & 205 & 39.6 & 9.3 & -126 & 0.31 \\
\hline 7 & Mozambique & Sofala & 2003 & 205 & 48.6 & 7.4 & -152 & 0.28 \\
\hline 8 & Kenya & Nyanza & 2003 & 206 & 33.4 & 3 & -152 & 0.40 \\
\hline 9 & Mozambique & Tete & 2003 & 206 & 54.8 & 2.5 & -152 & 0.28 \\
\hline 10 & Mozambique & Niassa & 2003 & 206 & 50.5 & 2.4 & -152 & 0.28 \\
\hline 11 & Guinea & Kankan & 2005 & 207 & 46.1 & 15.6 & -126 & 0.21 \\
\hline 12 & Ghana & Upper West & 2003 & 208 & 36.3 & 15.4 & -133 & 0.34 \\
\hline 13 & Guinea & Kindia & 2005 & 211 & 39.2 & 8.2 & -126 & 0.21 \\
\hline 14 & Burkina Faso & Cascades & 2003 & 211 & 45.8 & 30 & -166 & 0.22 \\
\hline 15 & Burkina Faso & Centre-Ouest & 2003 & 213 & 43.3 & 20.2 & -166 & 0.22 \\
\hline 16 & Niger & Tahoua & 2006 & 214 & 51.3 & 12.9 & -158 & 0.29 \\
\hline 17 & Niger & Dosso & 2006 & 215 & 46.7 & 11.5 & -158 & 0.29 \\
\hline 18 & Guinea & N'Zérékoré & 2005 & 218 & 45 & 11.9 & -126 & 0.21 \\
\hline 19 & Mozambique & Nampula & 2003 & 220 & 47 & 9.6 & -152 & 0.28 \\
\hline 20 & Malawi & Mulanje & 2004 & 221 & 53.1 & 8 & -109 & 0.18 \\
\hline 21 & Mali & Koulikoro & 2006 & 222 & 39.1 & 16.2 & -154 & 0.25 \\
\hline 22 & Mali & Mopti & 2006 & 227 & 40.9 & 12.7 & -154 & 0.25 \\
\hline 23 & Mali & Tombouctou & 2006 & 229 & 43.9 & 16.5 & -154 & 0.25 \\
\hline 24 & Burkina Faso & Nord & 2003 & 231 & 41 & 24.6 & -166 & 0.22 \\
\hline 25 & Niger & Maradi & 2006 & 231 & 66.4 & 14.3 & -158 & 0.29 \\
\hline 26 & Ruanda & East & 2005 & 233 & 47.5 & 4.6 & -109 & 0.20 \\
\hline 27 & Mali & Sikasso & 2006 & 237 & 45.2 & 15.8 & -154 & 0.25 \\
\hline 28 & Chad & Zone5 & 2004 & 240 & 45.6 & 15.9 & -122 & 0.20 \\
\hline 29 & Mozambique & Cabo Delgado & 2003 & 241 & 63.2 & 4.8 & -152 & 0.28 \\
\hline 30 & Zambia & Luapula & 2001 & 248 & 63.9 & 5.3 & -118 & 0.22 \\
\hline 31 & Chad & Zone7 & 2004 & 256 & 40.5 & 11.3 & -122 & 0.20 \\
\hline 32 & Nigeria & North East & 2003 & 260 & 48 & 10.8 & -166 & 0.36 \\
\hline 33 & Mali & Segou & 2006 & 262 & 40 & 14.6 & -154 & 0.25 \\
\hline 34 & Nigeria & North West & 2003 & 269 & 59.5 & 14.6 & -166 & 0.36 \\
\hline 35 & Niger & Zinder & 2006 & 269 & 65.1 & 15.9 & -158 & 0.29 \\
\hline 36 & Burkina Faso & Sahel & 2003 & 285 & 53.9 & 21.2 & -166 & 0.22 \\
\hline
\end{tabular}

Source: Authors' calculations based on latest DHS surveys of respective countries Note: $C V$ relates to the coefficient of variation and Range to the difference between the minimum and maximum value within a country. Both measures refer to under-five mortality rates. 
Figure 3.1: Stunting rates in SSA

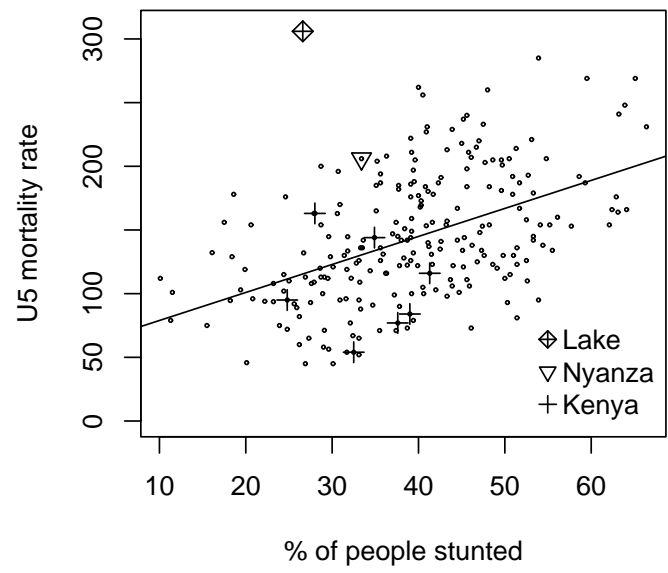

Source: Authors' calculations

Figure 3.2: Wasting rates in SSA

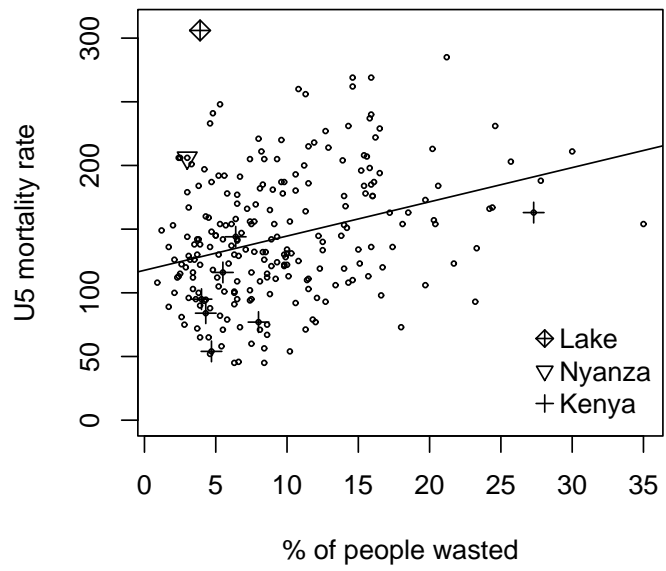

Source: Authors' calculations 
seem to be similar among all provinces bordering Lake Victoria, one possible explanation for this finding points to the role of the government and racial cleavages affecting migration decisions and the provision of health care. Compared to Uganda and Tanzania internal migration on provincial level in Kenya is limited by the prevalence of strong ethnic reservations. Furthermore, as discussed under section 3.4.3 discriminatory practices against the local ethnic group seems to have led to an underprovision of health care services in Nyanza compared to most other Kenyan provinces. In contrast, in Uganda the capital Kampala is situated in close proximity to Lake Victoria and therefore we would not expect an underprovision of health care services on the Ugandan side of Lake Victoria. Regarding Tanzania ethnic based inequalities in the provision of health care services are rather low due to the pursued nation building policies and hence health care provision seems to be much more need oriented (Miguel, 2004).

The simultaneous appearance of very low levels of malnutrition together with tremendously high rates of mortality in Nyanza and in particular the Lake Victoria region is unique in the SSA context. Moreover, the appearance of this phenomenon in a national context of relatively low mortality rates is further puzzling and led us to call it 'The Paradox'. Trying to explain this paradox is the objective of this paper and will demand a detailed review of the particularities of the Kenyan context.

\subsection{Theoretical Framework}

In order to analyze the described paradox we estimate reduced forms of child health and mortality production functions. The choice of our theoretical model relies on earlier work in this field done by Akin et al. (1992); Rosenzweig and Wolpin (1988) and in particular Behrman and Deolalikar (1988). An overview on the general relationships of underlying (exogenous) and proximate factors affecting health and mortality outcomes is presented in Figure 3.3 which is guided by the frameworks as outlined in Mosley and Chen (1984) and UNICEF (2008). The conceptual core of the framework is the idea that all background variables (cultural, socioeconomic or geographic) have to operate through a limited set of proximate determinants (environmental contaminations, maternal factors, infant feeding habits, and preventive health care practices) which in turn directly influence the risk of disease and the outcome of the disease process.

The relationship among health inputs and child health outcomes can be written as follows:

$$
H_{i j k}=H\left(E_{i}^{o}, P_{i}^{o} \text { jonk } v_{k}, u_{j k}, \varepsilon_{i j k}\right) \text {, }
$$


Figure 3.3: Theoretical Framework

Exogenous Factors (E)

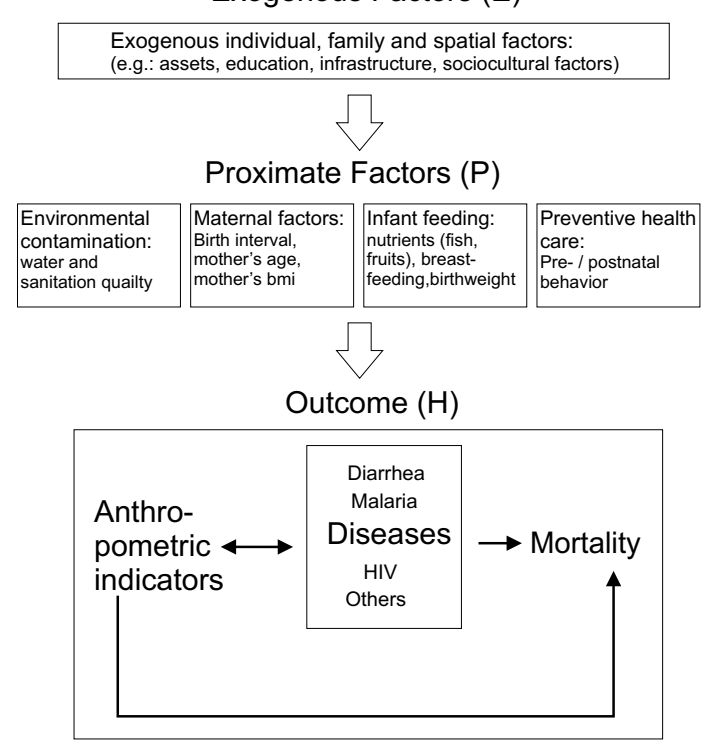

where the health of individual $\mathrm{i}$ in household $\mathrm{j}$ and community $\mathrm{k}\left(H_{i j k}\right)$ is produced by observed underlying factors $\left(E_{i}^{o}\right)$, observed proximate factors $\left(P_{i}^{o}\right)$ and certain unobserved underlying and proximate factors $\left(v_{k}, u_{j k}, \varepsilon_{i j k}\right)$.

Further on, equation 3.2 depicts the mortality production function for individual i, with mortality $\left(M_{i j k}\right)$ resulting if health falls below some critical level $H^{*}{ }^{2}$

$$
M_{i j k}=M\left(H_{i j k}-H^{*}\right)
$$

In the empirical analysis, the estimation of parameters for the health and mortality production functions can suffer from three types of problems. First of all, since all health related input variables are treated as exogenous, a bias might arise if we fail to control for simultaneously determined health inputs in the estimation of the health production function. Secondly, health and mortality outcomes are influenced by several individual, family, and community variables. Some of these variables can be observed; others cannot. Table 3.2 shows how the observed and unobserved factors can be classified in our particular case. The simple association between, for example, the stunting score of a child and the mother's educational level holds if the observed indicator (e.g. educational status) is not correlated with

\footnotetext{
${ }^{2}$ Empirically we will model $\mathrm{M}$ as the risk of mortality at time t.
} 
Table 3.2: Classification of Variables Influencing Health and Mortality Outcomes

\begin{tabular}{|c|c|c|}
\hline Variables & 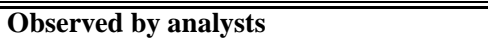 & Unobserved by analysts \\
\hline Individual & $\begin{array}{l}\text { Health indicators (anthropometric and, } \\
\text { mortality outcomes, reported diarrhea) } \\
\text { Health related practices (birth interval, } \\
\text { Clinical birth, caesarian section, duration } \\
\text { of breastfeeding, Retro-viral drugs } \\
\text { Death of previous child, vaccinations) } \\
\text { Age, gender, twin status }\end{array}$ & $\begin{array}{l}\text { Genetic endowment, } \\
\text { HIV status, } \\
\text { Nutritional intake }\end{array}$ \\
\hline Family & $\begin{array}{l}\text { Mother's education in years, } \\
\text { Mother's age at birth, marital status, } \\
\text { Mother's BMI and HIV status, } \\
\text { Household size, asset possession, } \\
\text { Ethnic belonging, } \\
\text { Water and sanitation access }\end{array}$ & $\begin{array}{l}\text { Genetic factors, Innate ability } \\
\text { for child care, Parental time } \\
\text { devoted to child care, General } \\
\text { knowledge and mental capability, } \\
\text { Intra-household resource allocation, } \\
\text { Income and expenditure levels }\end{array}$ \\
\hline Community & $\begin{array}{l}\text { Geographic location } \\
\text { Rural or urban }\end{array}$ & $\begin{array}{l}\text { General disease and health } \\
\text { environment, Public Infrastructure } \\
\text { Labor market conditions } \\
\text { (availability and quality of education } \\
\text { and health care, facilities, roads,...), } \\
\text { Water quality, Cultural habits }\end{array}$ \\
\hline District & Malaria suitability & Political factors \\
\hline Province & $\begin{array}{l}\text { Health access (People per Physician, } \\
\text { Health expenditures per capita) }\end{array}$ & Political factors \\
\hline
\end{tabular}

unobserved variables (such as labor market conditions) that affect the stunting score. However, if the unobserved factor affects the child's stunting status and is correlated with the educational attainment of the mother, the estimated effect is biased together with false standard errors for our parameter estimates. Thirdly, our obtained coefficients can be biased and our standard errors can be false, even if the unobserved factor affects only the outcome variable but is completely uncorrelated with the observed explanatory variables. This might be the case if in the incidence of clustering the mortality risk among siblings and among children residing in the same community is partially due to children sharing the same family and community characteristics. However, the correlation may persist after controlling for observed factors such that the remaining correlation is a consequence of genetic, behavioral, and environmental factors that are common to all children in a particular community or family but that are unobserved. As a consequence, the still correlated observations violate a standard assumption of independence in statistical analyses, resulting in standard errors that are understated and, in the case of non-linear models such as a hazard models, parameter estimates that are both biased and inconsistent (Trussel and Rodriguez, 1990). 
To mitigate the problems we adopted 3 strategies. First of all, in order to circumvent the first problem we estimate reduced form regressions and therefore exclude any explanatory variable that we would expect to cause such a problem, which in our case results in dropping the length of breastfeeding from our regression equations. Secondly, we construct and include malaria, health care and a Lake Victoria variable, indicating whether a child lives within $20 \mathrm{~km}$ around Lake Victoria in order to better capture the specific nutrition and disease environment as explained in more detail in section 3.4. Thirdly, as explained more in detail in section 3.5, we use mixed model representations to further address unobserved heterogeneity issues on the family and community level.

\subsection{Geography vs. Ethnicity: The Kenyan Context}

The seeming disconnection of anthropometrical indicators on the one hand and health and mortality patterns on the other and the prevailing huge spatial differentials in mortality and undernutrition indicators in Kenya requires a profound investigation of the underlying causes of this phenomenon. Moreover, in order to facilitate the understanding of the country specific context it is further useful to distinguish between underlying causes that have an effect on either child malnutrition or child mortality and those that have an effect on both of them simultaneously. Despite reviewing all of the relevant causes in this section we will only pay attention to those that are particular to the Kenyan context with a main focus on nutritional, epidemiological and cultural factors.

\subsubsection{Nutritional Environment}

Food and nutrition availability affects anthropometric and mortality outcomes likewise. While insufficient food and vitamin intake mostly influences child mortality indirectly by increasing the predisposition to diseases it often expresses itself directly in anthropometric measures which therefore frequently serve as proxies of the health and mortality environment in the absence of reliable data on the latter ones. The two most widely used anthropometric indices regarding children are stunting (low height for age) and wasting (low weight for height) which both serve a different purpose. Stunting is claimed to be an indicator of chronic undernutrition resulting of prolonged food deprivation or illness, meanwhile wasting is supposed to reflect acute undernutrition as a result of more recent food deprivation or illness (Nandy et al., 2005).

In addition to this there exist further factors that manifest themselves in both anthropometric indicators very differently. In particular the type of food consumed in the first months of life plays an important role in the growth process of 
children and hence affects the stunting indicator while the wasting indicator remains relatively unchanged. For the transformation of energy into body growth, certain micro- and macronutrients are very essential like iron, calcium, iodine, vitamin A or proteins (Moradi, 2009). Accordingly, the body on the one hand fails to grow at a normal rate as a result of chronically insufficient intakes of certain types of micro- and macronutrients and on the other hand exceeds normal growth rates if certain micro- and macronutrients are consumed in high amounts. An important food that makes a vital contribution to the survival, health and body growth of children is fish which provides quality proteins and fats (macronutrients) and vitamins and minerals (micronutrient). Furthermore, it is notable that not fish consumption per-se drives the higher than average growth process. Biomedical research shows that the growth effect due to fish consumption does only seem to occur in combination with a well balanced compositional diet (Marques et al., 2008).

Neglecting the role of diseases on stunting and wasting we would expect that both indicators show high z-values in areas where food availability is high. Moreover, the incidence of stunted children should be particularly low in those regions where fish is widespread available together with other crops and foods.

The Lake Victoria region in fact offers the advantageous conditions just mentioned. Soils are mostly of good quality resulting in agricultural production surpluses what led Fearn (1961) to call the region the 'granary of East Africa'. Moreover, fish is largely available in at least close proximity to the lake despite the strong export orientation of the fishing industry in the context of the nile perch boom. Moreover, the region is situated on the trade route between Tanzania and economically comparatively prosperous Central Kenya which might further help to increase the variability and availability of food in the region.

In general reliable administrative data on soil quality, frequency of rain and agricultural production for Kenya is not available. In the absence of these data it is difficult to asses the food availability and security situation for other regions in the country. Fortunately, USAID created the Famine Early Warning System (FEWS) that issues early warning and vulnerability information on emerging and evolving food security issues in the world. To inform researchers and policy makers FEWS generates the so called Water Requirement Satisfaction Index for maize (WRSI) and the Normalized Difference Vegetation Index (NDVI). Both indices are updated regularly and allow for the investigation of intra-country differences (fews.net). The WRSI for Kenya is used as an indicator of maize performance based on the availability of water to the crop during the growing season. Maize has been selected since it is the most important cereal crop in Sub-Saharan Africa and due to its properties to be cheaper, less water intensive and climatical more robust than other cereals which makes the WRSI an ideal indicator of food security. Looking at the distributional map of the WRSI for Kenya, Figure 3.4, two 
things are remarkable. First of all, the areas close to Lake Victoria obtain the highest scores for the whole country indicating a very food secure situation. Secondly, the level of food security deteriorates steadily the further a location is away from Lake Victoria an exception being the coastal area around Mombasa where the food security situation improves again (FEWS-Net, 2004). This result is reinforced by considering the map portrays of the NDVI for Kenya, Figure 3.5, which is based on meteorological NASA satellites using advanced very high resolution radiometer in order to indicate the vigor and density of vegetation at the earth's surface. An inspection of the map shows a similar pattern with the areas around Lake Victoria showing the highest vegetation density and areas further away depicting continuously decreasing vegetation levels (FEWS-Net, 2004). Relying

Figure 3.4: Water Requirement Satisfaction Index (WRSI)

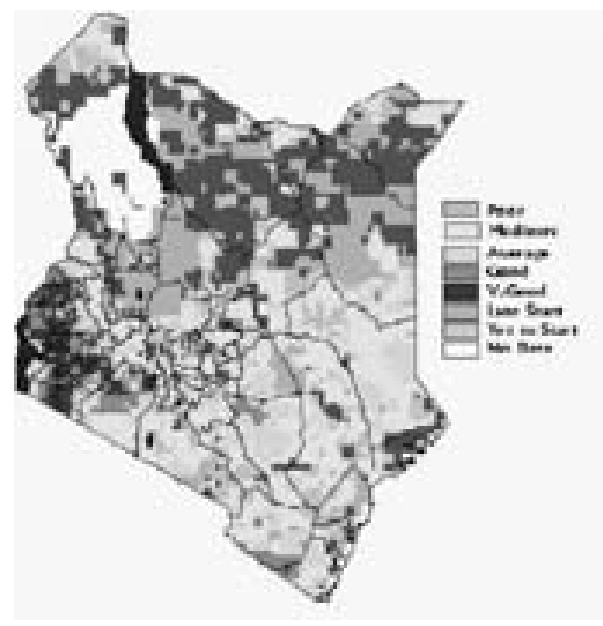

Source: FEWS-Net (2004)

on the two proxies for food security described above, two main implications can be derived from the previous considerations. Due to a general increase in food security and food availability the closer one gets to Lake Victoria, wasting and stunting indicators should show improvements reaching their lowest values in the area around Lake Victoria. Furthermore, the incidence of stunting should be extraordinary low on the shores of Lake Victoria since fish is widely available as a staple food around this area. 
Figure 3.5: Normalized Difference Vegetation Index - Kenya

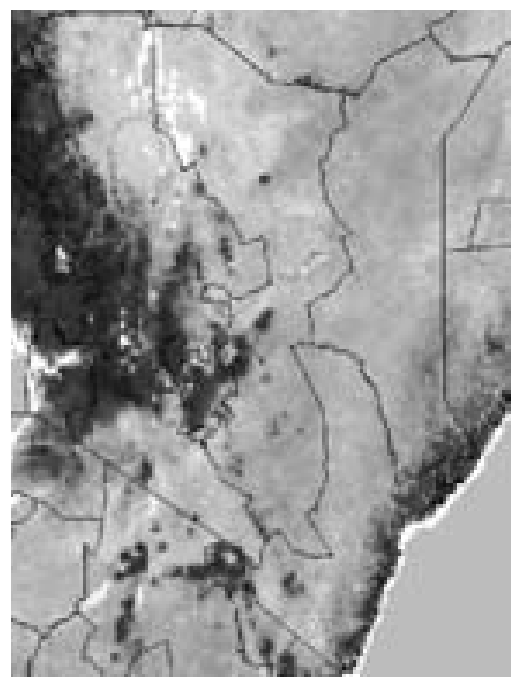

Source: FEWS-Net (2004)

\subsubsection{Epidemiological Factors}

Child mortality levels in Kenya declined rapidly after its independence in the early 1960s and reached its minimum levels in the late 1980s. From then on the trend reversed and despite a significant drop in overall fertility rates, mortality levels were continuously increasing up to 115 per 1,000 in the 2003 DHS round (Hill et al., 2004; CBS, 2004). This adverse trend was accompanied by stagnant growth of per capita income, declining levels of immunization, falling school enrollment, and foremost the emergence of the AIDS epidemic (Hill et al., 2004). One of the salient findings of the 1998 and 2003 DHS rounds is the enormous variation of child mortality rates among the provinces reaching 54 per 1,000 in the Central Province and 204 per 1,000 in Nyanza in the 2003 round.

In the following we want to shed some light on the principal underlying causes of the geographic differentials in the observed child mortality rates in order to underscore the role of Lake Victoria on mortality patterns in Kenya.

When looking at the data for Nyanza it surprises that the established link between a good nutritional status, in particular wasting or acute malnutrition, and child mortality risks is not reflected in the health statistics. While children in the Nyanza region show above average scores in the anthropometric indicators, infant and child mortality rates are highest in the region. A first major reason that helps Johannes Gräb - 978-3-653-00480-9 
to explain this paradox is the unequal distribution of malaria prevalence within the country - as depicted in Figure 3.6 - due to regional variations in temperature, humidity, and the existence of bodies of water. Although malaria is epidemic in several areas in Kenya, the Lake Victoria region is the only endemic region in the country with a transmission period that lasts over the whole year (MARA, 2004). Moreover, it is important to note that the risk of malaria infection does not decline in a continuous way starting from Lake Victoria. Due to the elevations of the East African rift valley arising only some kilometers away from Lake Victoria a natural malaria barrier exist in the east that drastically reduces the risk of malaria infection in these regions. In all other regions, except a small stripe on the coastal area, climatical conditions do not favor the reproduction of female anopheles mosquitoes over the whole year due to long periods without rain in these areas. Hence, this restricts malaria transmission rather to the rainy seasons in these areas.

Figure 3.6: Climate Suitability for Endemic Malaria

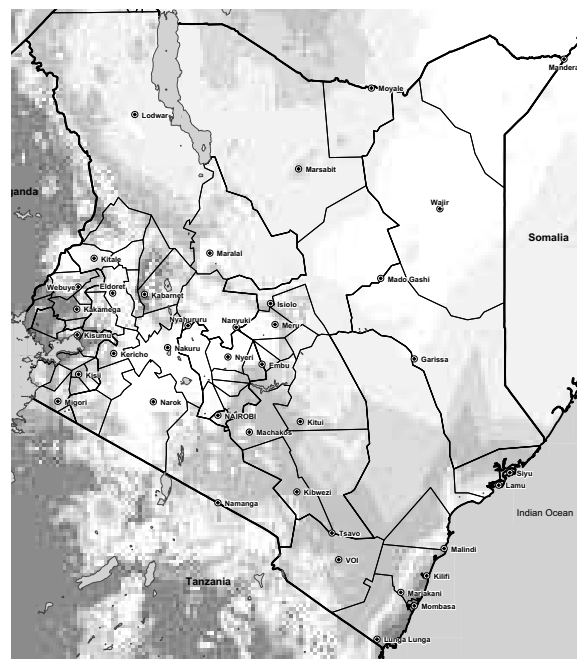

Source: MARA (2004)

A second major aspect that affects health and mortality outcomes of young children is the quality of drinking water. Despite being the second largest fresh water lake in the world, the water of Lake Victoria is not safe for drinking and several cases of outbreaks of waterborne diseases are reported each year (Ochumba and Kibaara, 1989; Oguttu et al., 2008; Omwega et al., 2003; Scheren et al., 2000).

The third major difference of the Lake Victoria region compared to other regions in Kenya is the high prevalence of HIV/Aids in the area. While cultural Johannes Gräb - 978-3-653-00480-9 
factors, as described later on, can explain part of the high HIV rates in the area, recent studies point to the social erosion of family norms among people around the shores of Lake Victoria. The nile perch boom in the area, starting in the mid 1990s, and the resulting demand for male labor forces in the fishing industry led to a strong influx of migrants into the region which was accompanied by a growing prostitution business (Geheb et al., 2008). Moreover, the increase in demand for male labor shifted the intra-household bargaining power towards men and contributed to weaken the already inferior position of women thereby increasing the likelihood of involuntary risky sexual behavior for women (Béné and Merten, 2008). Further on, Nyanza province is situated on thriving trade and migration routes connecting the economically powerful central area of Kenya with Tanzania. Together with the high urbanization rates in the Nyanza this is likely to contribute to the higher HIV/Aids rates in the area (Oster, 2008).

Taking into account the spatial distribution of mortality drivers as outlined above, we would expect strongly increasing mortality rates in close proximity to Lake Victoria the main reasons being among others the comparatively high HIV/AIDS prevalence and the much stronger predisposition to infectional diseases like Malaria in that area.

\subsubsection{Cultural Factors}

Ethnic belonging affects mortality and undernutrition levels in Kenya through a variety of mechanism. Notably geographic and cultural factors in addition to the prevalent political economy play an important role with respect to health outcomes of certain ethnic groups in the country.

Since most ethnic groups in Kenya live spatially concentrated in a very particular region of the country, current administrative provincial boundaries were usually drawn based on the location of a certain ethnicity. The Lake Victoria region is part of the Nyanza province which is predominantly inhabited by the Luo ethnic group. Although Luo have several cultural practices in common with most other ethnic groups in Kenya, there exist three noteworthy differences.

Firstly, while most ethnic groups in Kenya practice male circumcision, Luo besides Turkana, and Itero, which represent only a small part of the population, are known for not being circumcised thereby substantially increasing their risk of HIV infection and HIV related mortality (Chesoni, 2006). Secondly, the type of nutritional intake differs compared to other ethnic groups. Having lived for already more than 400 years in close proximity to Lake Victoria (East African Living Encyclopedia), Luo have benefited from the beneficial food availability and protein situation, therefore showing significantly better mean height values for men in historical data compared to all other Kenyan ethnic groups (Moradi, 2009). Given the long time span of settlement close to the Lake Victoria and the Johannes Gräb - 978-3-653-00480-9 
historical data, one might speculate over emerging genetic differences among the Luo and other ethnic groups that lead to mean height advantages that manifest themselves already in nutritional indicators in early ages. Following the recent WHO study on the new child growth standard genetic factors seem to play a minor role in explaining the disparities in physical growth among children (WHO, 2006). ${ }^{3}$ Thirdly, fish consumption in Kenya is not only determined by availability aspects but as well by cultural habits. While Luo and some ethnic groups in the coastal area use fish as a staple food, it is viewed with considerable suspicion among ethnic groups in Central and Eastern Kenya (Oniang'o and Komokoti, 1999; Peters and Niemeijer, 1987).

With respect to differences in the position of women among Kenyan ethnic groups, no clear picture exists. While polygamy is common among most of Kenya's ethnic groups widow inheritance is practiced primarily by the Luo and certain smaller clans among the Luhya ethnic group, therefore weakening the role of women in these ethnic communities. In contrast, female genital mutilation is practiced by the majority of Kenya's ethnic communities, while only Luo, Turkana, Luhya and Iteso do not (Chesoni, 2006). Moreover, female education levels tend to be high among Luo women compared to other ethnic groups (Wainaina, 2006).

Furthermore, ethnic belonging plays a crucial role on the allocation process of public resources and political positions in Kenya due to the prevailing kinship structures and patron-client relationships (Cohen, 1995; Miguel, 2004; Miguel and Gugerty, 2005; Weinreb, 2001) and this way affects health indicators. Out of the more than 40 ethnic groups in Kenya, the Luo represent about $13 \%$ of the Kenyan population and constitute the third largest ethnic group in Kenya whereby only the Kikuyu with $23 \%$ and the Luhya with about $14 \%$ tend to have higher shares in the overall population.

Although Luo ethnic groups took an important role in the independence process in Kenya, they have been politically under-represented at national political levels and except very recently not being part of any coalition since 1965. The Luo are the only major ethnic group in Kenya that has not been part of the national government since this time span and this under-representation of Luo interests on the national level has resulted in a limited access to public funds from the national level and lead to a steady under-investment of health and schooling facilities in the Nyanza region (Alwy and Schech, 2004; Muhula, 2008; Nyan-

\footnotetext{
${ }^{3}$ To further investigate this issue, we compared children's mean stunting scores between Luo and other ethnicities outside the Lake Victoria Region and additionally outside Nyanza and Western province. Based on an oneway ANOVA, differences in height for age scores turned out to be statistically insignificant for this setting. Therefore, we conclude that genetic differences do not explain the observed growth differential for children in our context.
} 
jom, 2006) compared to most other regions, the exception being the north east of Kenya.

Bearing in mind the circumcision behavior of ethnic groups in Kenya, we would expect the highest HIV/AIDS prevalence among the Luo ethnic group. In addition, the situation of the political economy in Kenya is likely to further aggravate the mortality levels of the Luo due to a worse access to health care facilities compared to the main other ethnic groups in Kenya (Cutler et al., 2006). Since discriminatory practices in the allocation process of public resources probably occurs in practice on a provincial level meaning that relatively less money will go to Nyanza as a whole, there might be an unfavorable effect on mortality levels for all ethnic groups living in Nyanza.

\subsection{Empirical Findings}

\subsubsection{Data}

\section{The KDHS 2003}

In the empirical analysis we use data from the 2003 round of the Kenyan Demographic and Health Survey (KDHS). The KDHS 2003 includes full birth history information from 4346 women of reproductive age that gave birth to at least one child in the five years preceding the 2003 KDHS survey. For the first time the KDHS includes data from all provinces of Kenya as well as data on HIV testing. Moreover, the survey is based on a two-stage survey design. In the first stage 400 clusters were randomly chosen from a master frame. Afterwards, households were systematically sampled out of each cluster. ${ }^{4}$ In every second household sampled, men, aged 15 to 54 years, were interviewed to conduct a Men's questionnaire. All women and men living in households selected for the Men's questionnaire were asked to voluntarily participate in the HIV testing. $76 \%$ of all eligible women voluntarily agreed to undergo the test. ${ }^{5}$

In addition to the variables directly derived from the household questionnaires, we calculate the distance of each cluster to the shores of Lake Victoria using the GPS coordinates provided by ORC Macro. We define the Lake Victoria region as the area within a $20 \mathrm{~km}$ boundary to the shores of the lake. Furthermore, we exploit the MARA (2004) database on endemic malaria to obtain district level

\footnotetext{
${ }^{4}$ In the following we often refer to clusters as communities since in the DHS context it is a geographical unit, consisting of several households.

${ }^{5}$ The official KDHS 2003 report provides several descriptive and multivariate examinations on whether non-participation in HIV testing is systematically related to other variables. No systematic relationship was found (CBS, 2004) and therefore we expect our results not to be effected by sample selection bias when using the reduced HIV sample.
} 
information on malaria prevalence in Kenya. ${ }^{6}$ Unfortunately, we could not obtain data on the health care sector in Kenya on the district level. Instead, we rely on data published in Nyanjom (2006) who reports information on the number of people per medical officer and public health expenditures per capita on provincial level for the time period 1995 - 1998.

\section{The samples}

As is common in the literature we include only those children who were born within the 5 years preceding the survey. Since hygienic and socio-economic conditions are less likely to have changed over the course of 5 years compared to 10 or more years, this decision improves the accuracy of the matching of the covariates to the actual survival time in the multivariate analysis of under-5 mortality. Moreover, data on children's height and weight has only been collected for those children being below the age of 5 at the time of the survey therefore restricting the information on the nutritional status of children to the same period of time.

The mortality sample consists of 1368 mothers reporting 2697 births in the last five years. 605 of these children died. The respective undernutrition sample remains with 1218 (1217) mothers who reported data on 1704 (1701) living children at the time of the survey for the stunting (wasting) regressions. ${ }^{7}$

\section{Variables of Interest}

The selection of variables for descriptive statistics and the undernutrition and mortality regressions is guided by the frameworks outlined in the previous section and the discussion of the role of ethnical, political and geographical factors in section 3.4 of this paper. An overview of variables used in this article including its coding is provided in Table 3.3.

\footnotetext{
${ }^{6}$ The same data base from http://www.mara.org.za/ was used in Oster (2007) to calculate regional malaria prevalence rates. Moreover, at the country level these malaria measures are closely correlated with climate-determined malaria susceptibility, as used in Sachs and Malaney (2002).

${ }^{7}$ Data on childrens' height and weight was missing for $11 \%$ of all living children. 


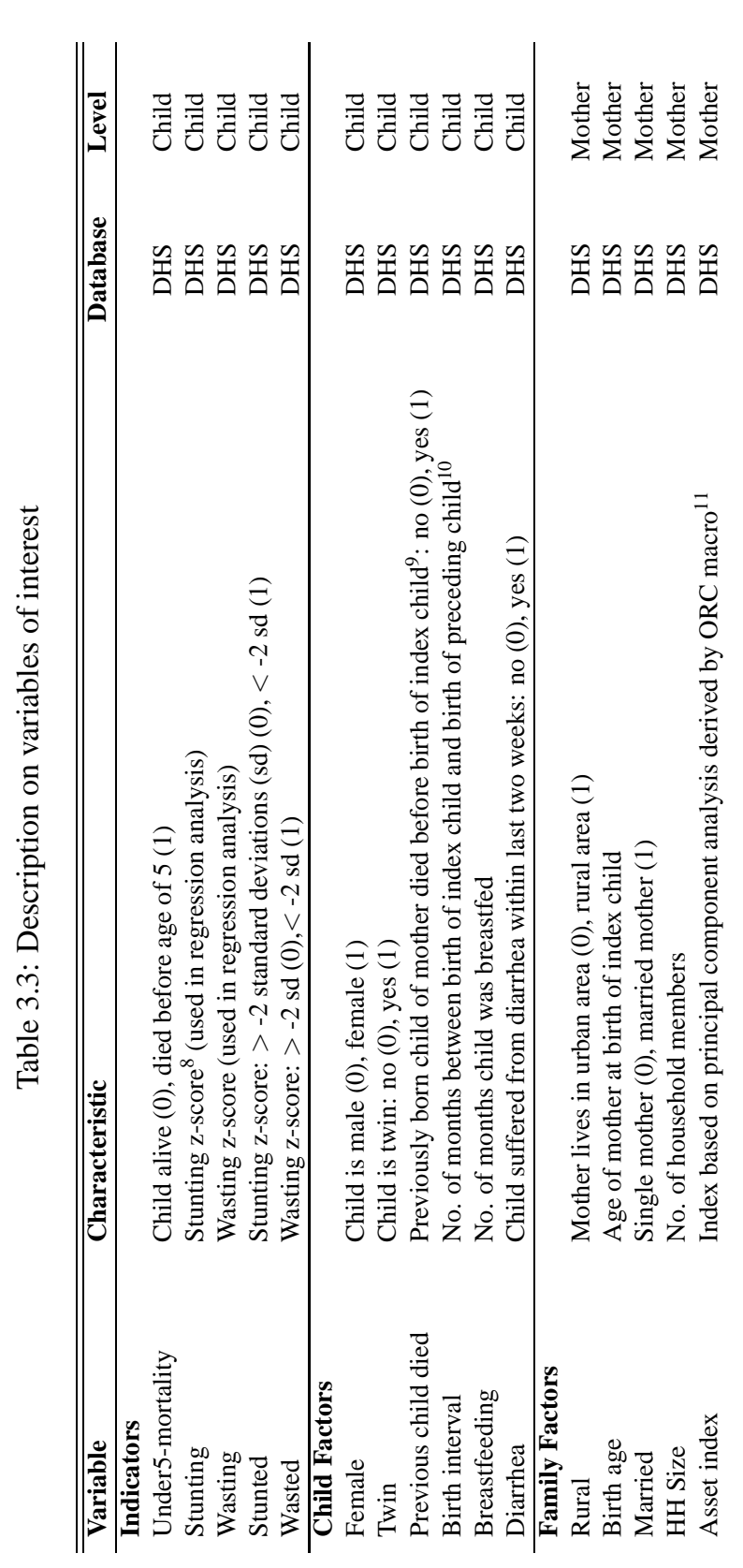

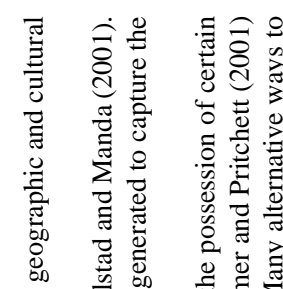

ソी $\quad$ की

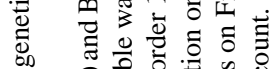

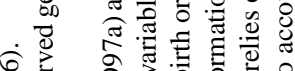

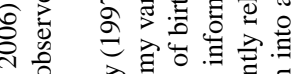

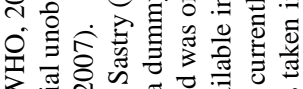

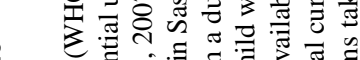

웧

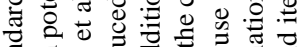

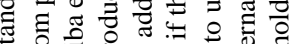

क

ช

可

링

ב

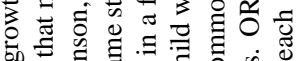

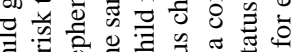

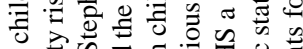

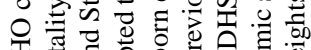

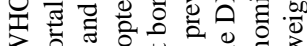

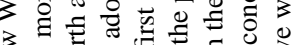
उั0

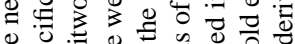

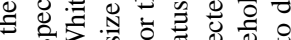

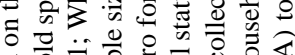

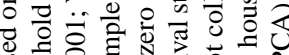

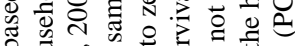

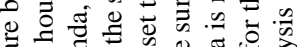

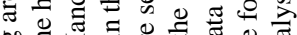

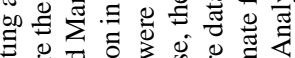

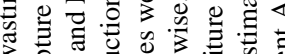

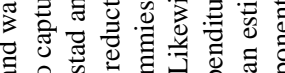

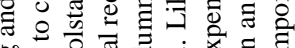

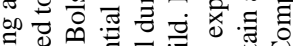

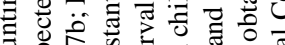

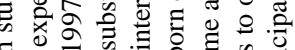
б.

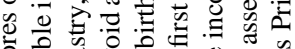
ठ . N

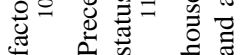




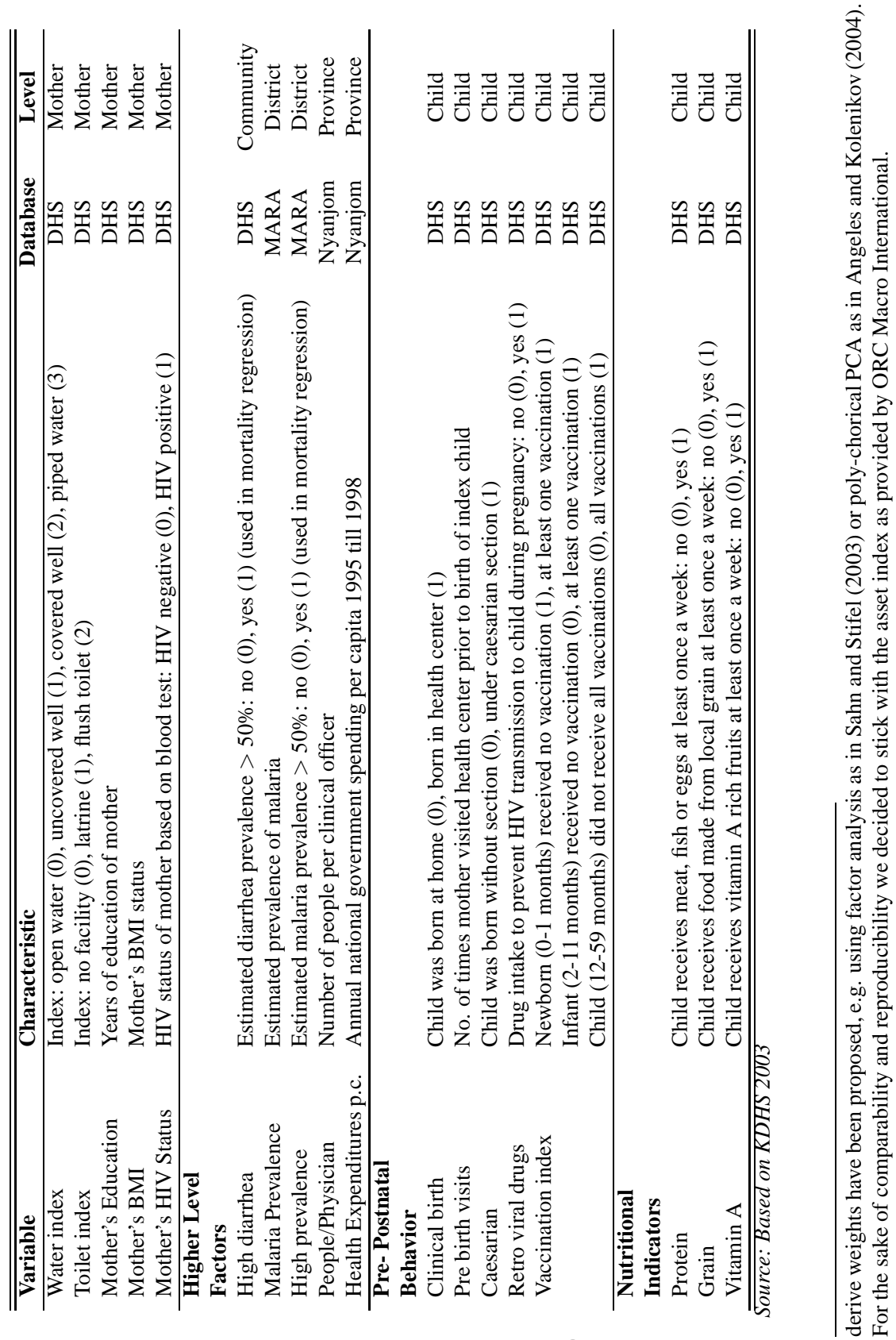


Two variables deserve particular attention. First of all, as mentioned earlier, we take the HIV status of the mother into account. The interpretation of this variable in the regression context is not straightforward. Since the HIV status of children has not been collected, it remains unclear whether the AIDS virus has been transmitted to the child at all during pregnancy or breastfeeding period and whether the mother already had the virus at the time of the birth of the child. Incorporating the HIV status of the mother in the regressions is therefore likely to yield a downward biased coefficient with a lower significance level. Moreover, the HIV status of the mother does not only measure a direct epidemiological effect on children but as well a socioeconomic one. In particular, children in a HIV affected household might suffer from diminishing capacities of their main caregivers to purchase certain key inputs for the children due to a loss of household income as a result from the disease. Furthermore, as described in section 3.4, the HIV status of a parent is partly related to cultural practices, e.g. male circumcision and therefore inhibits cultural elements as well. Secondly, the distance of a cluster to Lake Victoria plays an important role in our study. As pointed out in section 3.4 we would expect to see much better stunting values in close proximity to Lake Victoria due to the availability of fish and other food over the whole period of the year while with respect to wasting we would assume constantly improving wasting rates the closer Lake Victoria. In contrast, we would infer under-5 mortality levels to substantially deteriorate in close proximity to Lake Victoria due to the much higher disease environment in this area. Since most of our health environmental and geographical variables are either only on provincial or district level and moreover might not be free of measurement error, we would still expect to have an effect on our distance variables. In order to measure these effects appropriately we include a dummy variable indicating whether a household lives in the Lake Victoria region, within a distance of $20 \mathrm{~km}$ of Lake Victoria, in the stunting and under-5 mortality regressions while in the wasting regression the distance to Lake Victoria is incorporated as a continuous variable.

From the economic literature (Mosley and Chen, 1984; Smith and Haddad, 2002) as well as from our theoretical framework, it becomes clear that the variables described in Table 3.3 are important to study the determinants of undernutrition as well as the context of under-5 mortality. Thus, we use the same list of covariates in the multivariate analysis of undernutrition and mortality. ${ }^{12}$ The KDHS includes some further variables, e.g. information on children's protein intake or pre- and post natal care, which are only used for descriptive purposes since

\footnotetext{
${ }^{12}$ The final model specifications include squared terms whenever the respective coefficient showed a statistically significant value. Otherwise squared terms were excluded. In this regard model specifications might differ between the stunting, wasting and mortality regressions. 
these variables exhibit a very large number of missing observations leading to a too strong reduction in the sample size for the multivariate analysis.

\subsubsection{Descriptive Statistics}

Summary statistics on the variables used in this study are provided in Table 3.4. Moreover, we distinguish in Table 3.4 between different geographical and ethnical specifications. Column 1 depicts statistics based on the Lake Victoria region, the area within a $20 \mathrm{~km}$ distance of the shores of the lake, while column 2 provides information based on overall Kenya except the Lake Victoria region. Column 3 and 4 refer exclusively to the Lake Victoria region. In column 3 summary statistics are provided for the Luo ethnicity while statistics on the remaining ethnic groups in the area are shown in column 4. In addition, columns 5-12 comprise information on the same set of variables for overall Kenya and for each of the eight Kenyan provinces.

The first 3 lines of Table 3.4 already demonstrate the distinct setting of the Lake Victoria region in Kenya in terms of child malnutrition and mortality outcomes. As discussed in section 3.4, average rates in stunting and wasting are far below average in this region. Quite the contrary, under-5 mortality is by far the highest in Nyanza, reaching its peak in the Lake Victoria region. Bearing in mind that the underlying causes of malnutrition and mortality may differ, we look separately at geographical and ethnic disparities in the variables of interest. 


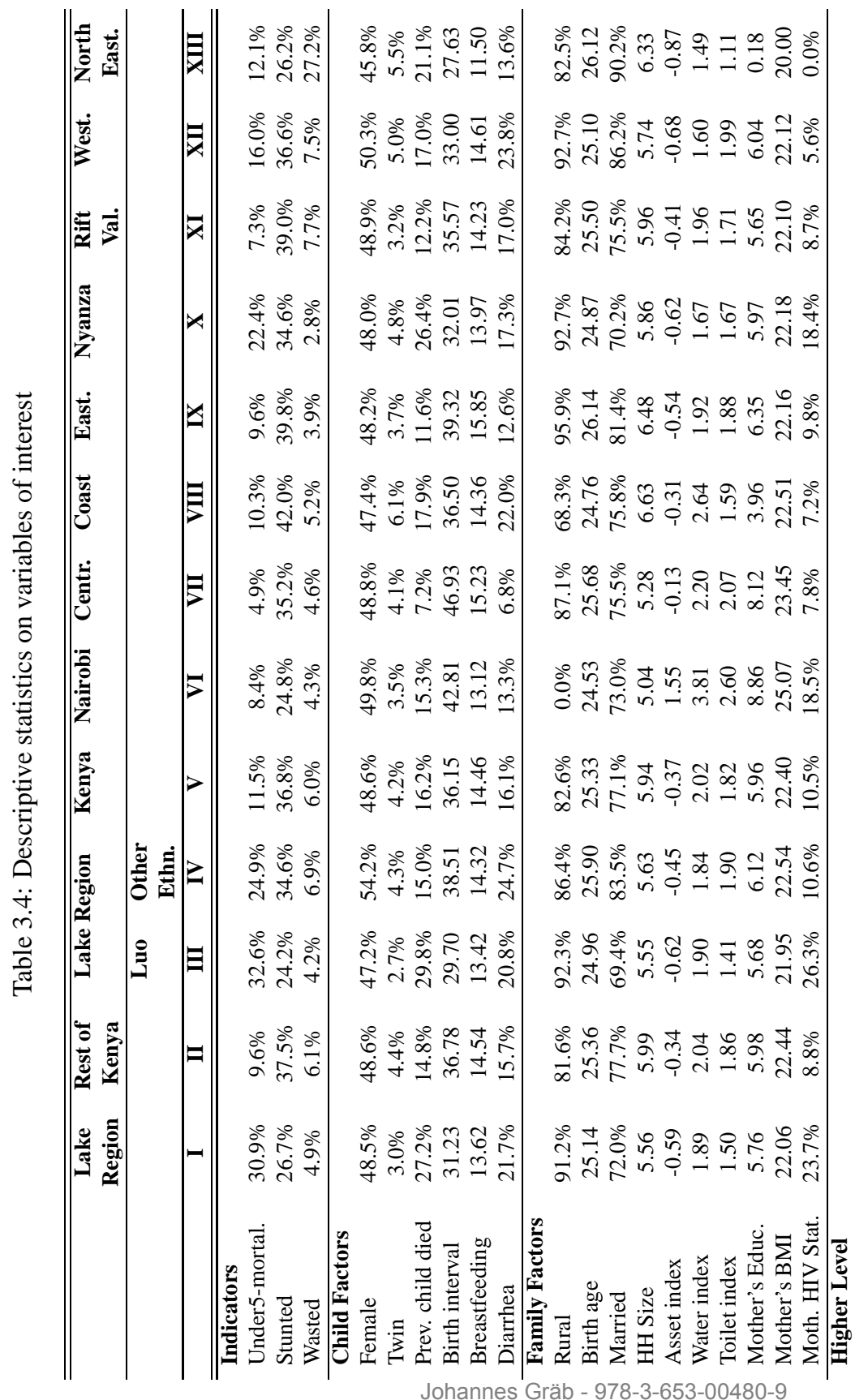

Downloaded from PubFactory at 01/11/2019 02:31:01AM 


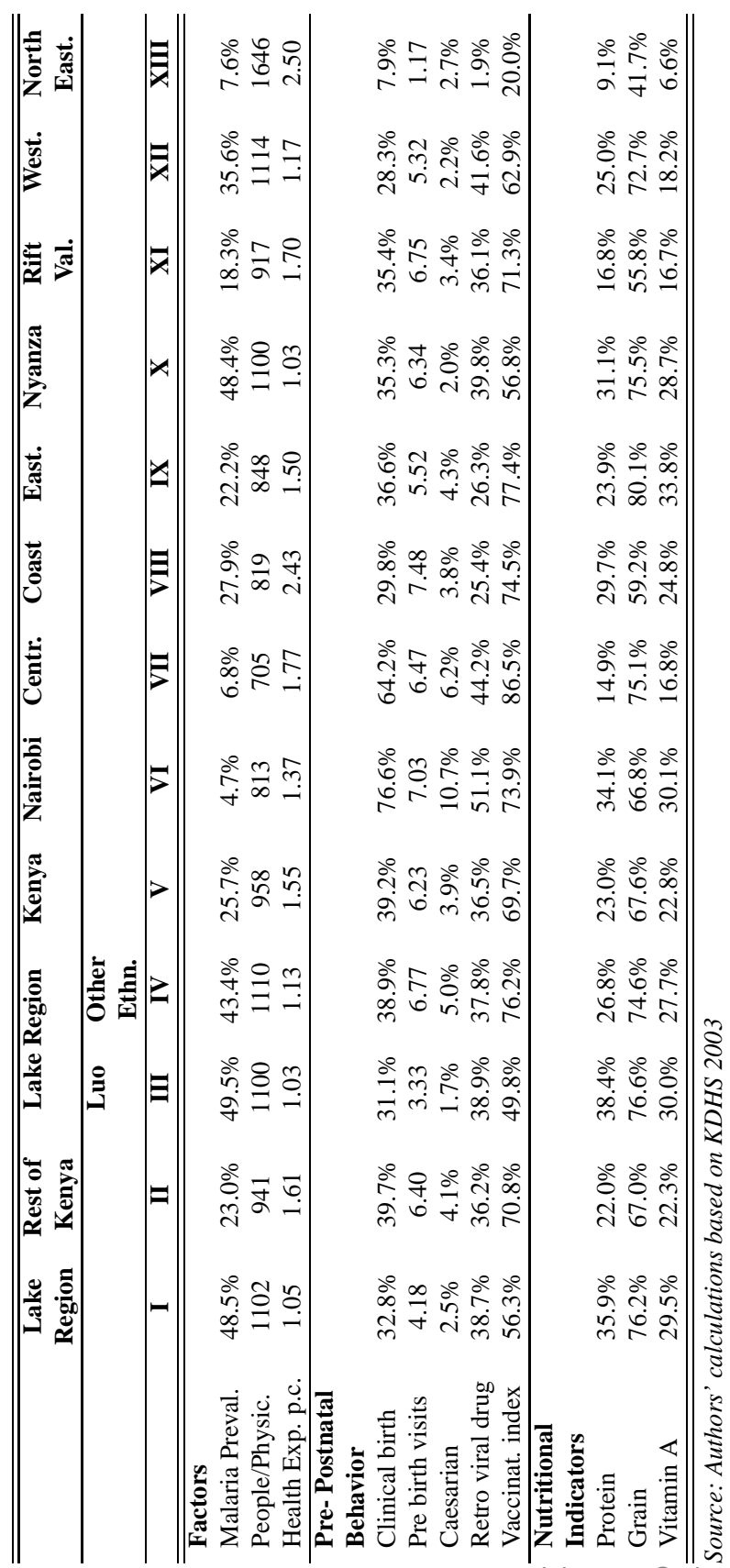




\section{Malnutrition}

The results from Table 3.4 show that the dietary intake is much higher in the Lake Victoria region than in the rest of the country, suggesting higher food availability at the shores of Lake Victoria. The observed higher than average intake of local grains and vitamin A rich fruits, like mango or papaya, can be attributed to the fertile soil found in the lake basin in combination with enough rainfall, facilitating a large supply of these aliments. Moreover, 38\% of Luo mothers in the Lake Victoria region allocate protein rich food to their children at least once a week, compared to only $26 \%$ of mothers belonging to other ethnic groups in the region and to a national average of $22 \%$. This remarkable difference between Luo and other ethnic groups within the Lake Victoria region might partly be explained by different food preferences as outlined in section 3.4.3 whereby other ethnic groups do not use fish as a staple crop. Figures 3.4 and 3.5 suggest more favorable agricultural conditions the closer the lake (FEWS-Net, 2004), substantiating the finding of highly cultivable soil near Lake Victoria. In the DHS, data on protein intake is not further disaggregated into its share of fish, meat or eggs. We use secondary data to stress the relevance and availability of fish. Data from the Kenya Integrated Household Budget Survey (KIHBS) 2005/2006 shows that fish consumption is highest in Nyanza province. In addition, households in Nyanza seem to spend $6.1 \%$ of their budget for food on fish compared to a national average of 2.1\% (Kenya National Bureau of Statistics, 2006).

\section{Mortality}

The high mortality rates in the Lake Victoria region point to the existence of extraordinary factors that help to explain the observed outcomes.

Table 3.4 depicts considerably higher malaria, HIV and diarrhea prevalence in the Lake Victoria region than in all other parts of Kenya. This result confirms the findings from the literature review in section 3.4. Moreover, high malaria rates are not only confined to the Lake Victoria region and Nyanza but to a lesser extent as well to Western province which might be indebted to some of its area bordering Lake Victoria. Furthermore, the incidence of diarrhea in the Lake Victoria region $(21.7 \%)$ is clearly above the Kenyan average and increases about $4 \%$ compared to the Nyanza average which might indicate the poor quality of drinking water of Lake Victoria and in its connected open waters. Further on, HIV rates in Nyanza and in particular in the Lake Victoria region are much higher than in any other area of the country which seems to be partly due to its comparatively high level of urbanization and its extraordinary position as a traffic hub between Tanzania and Central Kenya. 
Geography plays an important role on under-5 mortality. Ethnicity may be as important. The Lake Victoria region is - besides its unfavorable disease environment - characterized by being predominantly populated by the Luo ethnic group. The Luo represent $81 \%$ of the total Lake Victoria population and $94 \%$ of the Lake Victoria population in the province of Nyanza. The small upper northern part of the Kenyan Lake Victoria region belongs to Western province and is mainly populated by the Luhya ethnic group who also represent almost the entire remaining population of Nyanza (17\%). Distinguishing between ethnic groups within the Lake Victoria region, enables us to disentangle the effect of geographical and cultural factors. The Luo exhibit significantly worse outcomes in several proximate factors of child mortality than the other ethnic groups around Lake Victoria. $26 \%$ of all Luo mothers are tested HIV positive. Compared to a national average of $8.8 \%$ and to an average of $10.6 \%$ for the remaining ethnic groups in the Lake Victoria region, the result clearly points at a strong relationship between cultural habitus of the Luo and HIV infection, as already described in section 3.4. Moreover, the size of the differences between ethnic groups in the Lake Victoria region astonishes. While circumcision practices have been pointed out as one of the potential reasons for higher HIV rates among the Luo, the difference in most DHS reports for SSA countries between circumcised and uncircumcised adults was substantially lower and usually amounts to $4 \%-7 \%$.

A similar picture emerges from maternal factors and the pre and post natal behavior of the Luo. Luo mothers seem to start much earlier with bearing children than Luhya and most other ethnic groups. In addition, birth intervals between consecutive children are considerably shorter reflecting higher total fertility rates among Luo than among any other ethnicity (CBS, 2004). Moreover, short preceding birth intervals are often caused by the death of the previous child. Short succeeding birth intervals result in termination of breastfeeding. Indeed, both indicators, previous dead child and breastfeeding, are found to be especially under performing for Luo. Furthermore, the average number of child deliveries and caesarean sections as well as pre-birth visits in official health centers all show the lowest value for Luo. Besides of cultural habits of the Luo, these outcomes may also point to discriminatory practices against the Luo from the national level resulting in limited access to public funds and hence to lower public health expenditures. Such an interpretation is supported by the data on the Kenyan health care sector obtained from Nyanjom (2006). This data indicates that Nyanza receives the lowest amount of public health expenditures per capita and further on has the highest ratio of inhabitants per physician among all Kenyan provinces.

The descriptive findings are in line with the considerations undertaken in section 3.4. Thus, geographical, cultural and political factors seem to contribute jointly to the high mortality rates in the Lake Victoria region compared to the rest of Kenya. Due to the simultaneous occurrence of all three of these factors in the 
Lake Victoria region, it is difficult to establish the influence of a certain factor on our anthropometric and mortality outcomes when relying on bivariate statistics and analysis. Therefore in the following section, we will use regression analysis to examine causal relationships going from the observed covariates to the malnutrition and mortality outcomes, thereby putting a special emphasis on geographic and cultural factors.

\subsubsection{Method}

In order to investigate the determinants of undernutrition we rely on a linear regression model, while in the context of under-5 mortality we use a (non linear) Cox proportional hazard model. We will use multilevel extensions of the respective models for various reasons. Multilevel modeling allows for efficient and, for non linear models, consistent estimation in the case of significant intragroup clustering. Beyond, we will particularly exploit the variance decomposition, inherent in multilevel modeling. As we will see later on, the modeling helps in demonstrating the relevance of the family and community environment for individual outcomes and also to assess the contribution of observed covariates on the withinlevel variation.

The survey design of the KDHS 2003 involves hierarchical collection of data at the family and community level which results in clustering of undernutrition and mortality outcomes. In regression analysis, clustering is problematic if it is not only due to observed but also to unobserved household and community factors. For instance, net of observed factors, people within the same community are more alike than people across other communities since they are likely to share similar latent characteristics. Therefore, the statistical assumption of independence of the error term is violated and as a consequence confidence intervals are underestimated leading to false statistical inference. Typical unobserved factors at the family level are shared genetic factors, social practices or the pre and post natal behavior of the mother (Bolstad and Manda, 2001). Likewise, shared environmental factors may lead to clustering at the community level. ${ }^{13}$ To illustrate the extent of clustering in the KDHS 2003 data, we focus on the observed under-5 mortality outcomes. In our sample for all of Kenya 2915 (67\%) of the 4346 interviewed women did not experience any child deaths, while $941(21.7 \%)$ women had to suffer from the death of exactly one child. Just 184 (4.2\%) women experienced three or more child deaths in the five years preceding the survey. These $4.2 \%$ account for more than $30 \%$ of all deaths, showing a substantial amount of correlated outcomes and clustering within families. A similar pattern arises at the

\footnotetext{
${ }^{13}$ See section 3.3 or Sastry (1997a) for a detailed overview over potential unobserved family and community characteristics.
} 
community level. $62 \%$ of the 400 clusters under consideration contribute to $26 \%$ of all dead children, while on the other hand $21 \%$ of the communities account for more than $50 \%$ of all child deaths.

Due to the large clustering of outcomes on the mother as well as on the community level, we use multilevel mixed effects variations in the respective regression models. We use three-level models, controlling for correlated outcomes among siblings, i.e. on the household level, and among communities. ${ }^{14}$ In these models the error term is decomposed into a single error term on each level, capturing unobserved heterogeneity at each level. We will especially exploit the option of variance decomposition to measure the explanatory power of the covariates on the between family and community variation and, even more, to shed light on the specific contribution of each variable on the overall variation of malnutrition and mortality outcomes across families. ${ }^{15}$

\section{The linear multilevel model for malnutrition}

To analyze the driving factors of malnutrition, we use a linear multilevel regression model. As mentioned above multilevel models are applied to control for clustering caused by unobserved heterogeneity and to increase the precision of the estimated coefficients of the covariates. For an introduction into linear multilevel models see section 2.3.2.

Since we control for family as well as for community specific effects, our three level random intercept model reads:

$$
y_{i j k}=\left(\gamma+\beta_{1} x_{i j k}\right)+\left(v_{k}+u_{j k}+\varepsilon_{i j k}\right),
$$

with $i=1, \ldots, I$ individuals, $j=1, \ldots, J$ households and $k=1, \ldots, K$ communities. $U_{j k}$ is the household random effect, $v_{k}$ the community random effect. $Y$ is a vector of stunting and wasting outcomes, respectively and $X$ is a vector of observed covariates.

The models are estimated using the "xtmixed" command implemented in Stata (Stata, 2007).

\section{The multilevel Cox frailty model for under-5 mortality}

Cox proportional hazard models, proposed by Cox (1972), are the standard models used in child mortality analysis (Cox and Oakes, 1984; Cameron and Trivedi,

\footnotetext{
${ }^{14}$ Using a two-level model by neglecting correlations on the community level would lead to an overestimation of the family random effect.

${ }^{15}$ It should be noted that multilevel modeling may not control for omitted variable bias in case of linear models. Fixed effects models would be required. However, panel data on the respective household information is not available and panel estimation therefore infeasible. 
2005). In a proportional hazard model, the hazard rate is the instantaneous risk of death in $t$ conditional on survival up to $t$ :

$$
\lambda(t \mid x)=\lambda_{0}(t) \exp \left(x^{\prime} \beta\right) .
$$

$\lambda_{0}$ is the baseline hazard only depending on $t$. On the contrary, $\exp \left(x^{\prime} \beta\right)$ depends only on $x$. The idea is that all hazard functions are proportional to the baseline hazard, just being shifted by the scale factor $\exp \left(x^{\prime} \beta\right) . \lambda(t \mid x)$ is the hazard of child death at time $t$ given $x$. The advantage of the semi-parametric Cox proportional hazard model over parametric models is that no functional form of the underlying hazard function has to be assumed.

The multivariate kindred frailty model has been developed by Vaupel (1989, 1990). The two-level model was applied to study the effect of unobserved shared family characteristics on survival. Sastry (1997b) and later on Bolstad and Manda (2001) in a full Bayesian approach and Pankratz et al. (2005) extended the model to the multilevel case. ${ }^{16}$ In a standard frailty model, a frailty, $z$, is an unobserved random effect which works multiplicatively on the hazard function.

$$
h(t \mid x, z)=z \lambda(t \mid x) .
$$

In economic survival analysis, the frailty is usually referred to as a shared frailty since it is a random effect which is the same for all members of a group, for example a family or a community effect (Anderson et al., 2007). Transferred to the three level model with unobserved frailty on the household and community level, the hazard function reads:

$$
h_{i j k}\left(t \mid x_{i j k}, u_{j k}, v_{k}\right)=u_{j k} v_{k} \lambda\left(t \mid x_{i j k}\right),
$$

with individuals $i=1, \ldots, I, j=1, \ldots, J$ households and $k=1, \ldots, K$ communities. $U_{j k}$ is the household random effect, $v_{k}$ the community random effect. The individual (child) frailty is absorbed in the baseline hazard. The unobserved frailty is assumed to be independently distributed of all covariates and to follow a Gamma distribution with mean 1 and variance $\theta$ (Sastry, 1997b).

The family frailty effect measures the variation in family specific exposure to risk across families after controlling for observed variables. Children of families with a large frailty have, ceteris paribus, a larger risk of dying. This excess risk would be triggered by unobserved behavioral or genetic family specific factors. Insignificant variation of the family frailty effect would mean that there are no unobserved family specific characteristics. In this case, survival chances of siblings would be uncorrelated.

The model is estimated using the "coxme" command of the kinship package in R (Therneau, 2006).

${ }^{16}$ Guo and Zhao (2000) give a good overview over multilevel modeling for binary data. Johannes Gräb - 978-3-653-00480-9 


\subsubsection{Regression Results}

In all of our regressions, we start with a conventional single level regression specification, called Model I or the 'Standard Model'. In Model II a family random (frailty) effect is incorporated into the model. Finally, we add in Model III a community random (frailty) effect. Likelihood ratio tests are applied to test for significant random effects in the linear as well as in the non linear model (Goldstein, 2003). Since Model III performs - based on the likelihood ratio tests - significantly better than model II and I for all of our analyses, we will discuss our regression results based on this model. We will, however, compare the results of Model III with the inferior models to justify multilevel modeling.

\section{Undernutrition}

Results of stunting and wasting regressions are shown in Table 3.5 and 3.6, respectively.

\section{Stunting}

In Model III, stunting values are significantly better for girls than for boys which has been a common finding in the literature on SSA countries (Svedberg, 1990; Klasen, 1996; Wamani et al., 2007). Being a twin shows a significant negative effect which seems reasonable given the higher nutrition competition and the circumstance that twins are smaller and lighter at birth compared to single births. Children of better nourished mothers, as indicated by a higher body mass index, have, ceteris paribus, a higher height for age score. Moreover, the higher wealth, educational attainment of the mother, and the better the access to water, the better the stunting values of a child. On the other hand, the age of women at birth as well as living in rural areas, or having access to better sanitation facilities does not seem to have an effect. ${ }^{17} 18$

Interestingly, a child's disease status (hiv, diarrhea, malaria) does not have a significant impact on a child's height for age status. Diseases have a rather shortterm impact on children's health outcomes. The long-term indicator stunting may therefore fail in displaying any significant negative relationship.

\footnotetext{
${ }^{17}$ The significant positive coefficient of the variable "people per physician" is most likely driven by the tremendous share of people per physician in the North Eastern province which exhibits, however, very good stunting outcomes.

${ }^{18}$ Once again, standard errors of all estimates mentioned above slightly increase from Model I to Model III. The significance of the variables, first born child, birth interval and Luo vanishes when controlling for unobserved heterogeneity in the multilevel specifications. Neglecting highly correlated outcomes of children within families and communities leads to false statistical inference, justifying the usage of multilevel models.
} 
Children residing in close distance to Lake Victoria are showing significantly more favorable height for age outcomes. As pointed out in sections $3.2 \& 3.5 .2$ the high availability of fish in combination with very advantageous agricultural conditions are likely to be the main reason for this puzzling result and the extraordinary low prevalence of stunted children in the region.

These results endorse, once again, our afore mentioned theoretical considerations. Adverse geographic factors in the Lake Victoria region - malaria suitability, HIV endemicity and possibly unsafe drinking water - may affect the excessive risk of death for children in the region. They do, however, not affect a child's stunting status. Quite the opposite, the region around Lake Victoria exerts positive influences on children's nutritional outcomes. Fertile soils, a high level of food security and high protein availability (fish) foster the growth process of children. This result does not question the clear epidemiological relationship between a child's disease status and its mortality outcome. But the findings challenge the adequacy of inferring children's health and mortality outcomes from children's height status. Environmental factors - as found in the Lake Victoria region - can substantially modify the relationship between children's growth and health status.

\section{Wasting}

In contrast to stunting, wasting outcomes of children are much stronger affected by short term factors and, hence, are subject to much larger fluctuations. A poor wasting status is often a consequence of having recently suffered from illness or from insufficient food intake (Fawzi et al., 1997; UNICEF, 2008). As a consequence of these short term variations and missing information on important factors wasting regressions are usually not considered by demographers and economists. Technically speaking, a lot of the variation in wasting indicators among children is due to short-term variation, e.g. whether a child got sick or not, which can not be captured appropriately by the set of variables obtained from common household surveys. Therefore, determinants of anthropometric outcomes in wasting regressions are much more difficult to establish than in stunting regressions. Nonetheless, section 3.4.1 provides a priori reasons to believe that there are very crucial geographical factors at play that should be able to be verified even in the multivariate case. In particular, the observation that food insecurity constantly increases throughout the country, the further away Lake Victoria, demands to be investigated. Instead of using a dummy variable referring to the Lake Victoria region, we introduce a continues variable measuring the distance to the Lake Victoria in $\mathrm{km}$. Regression results are presented in Table 3.6. 


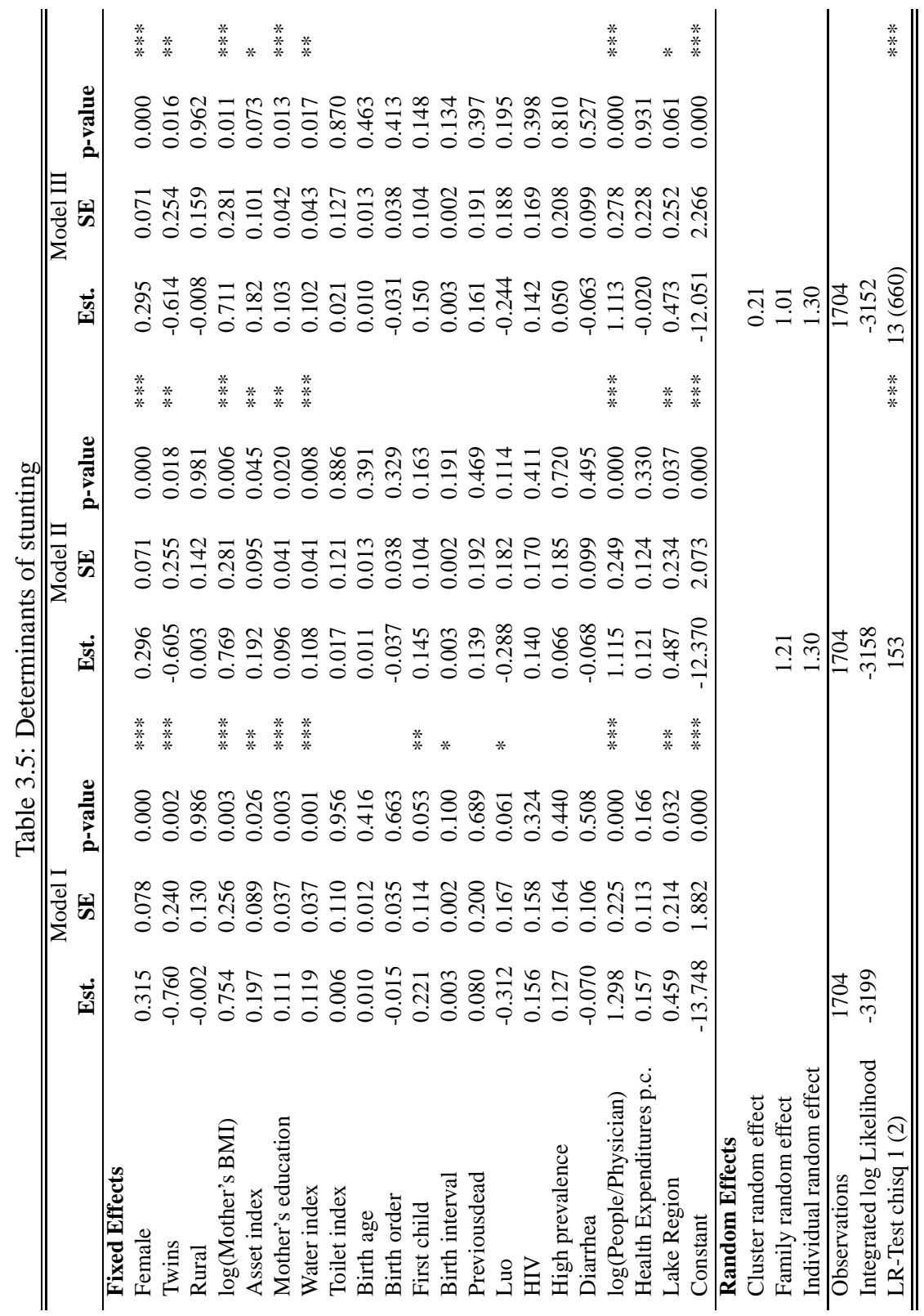




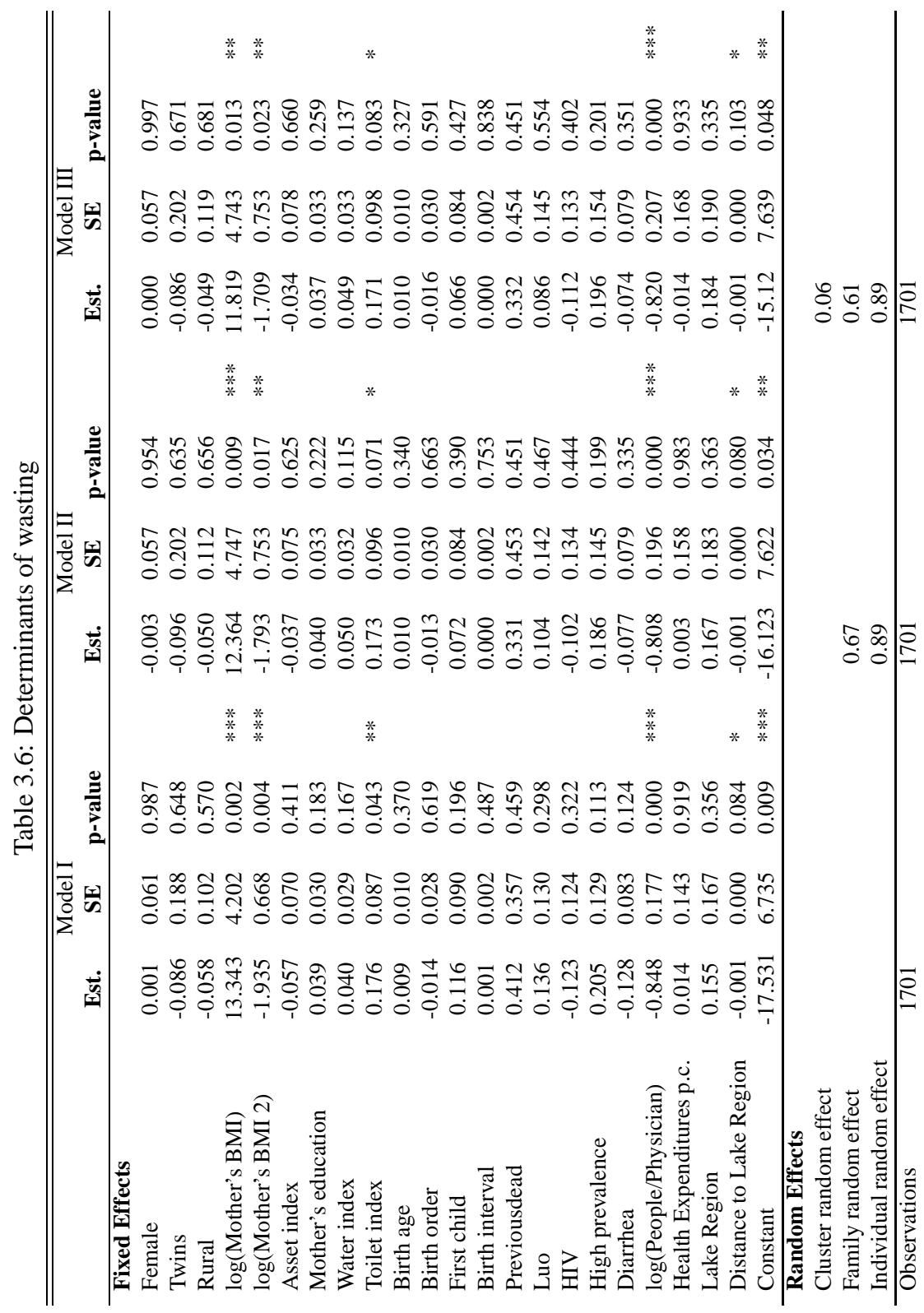




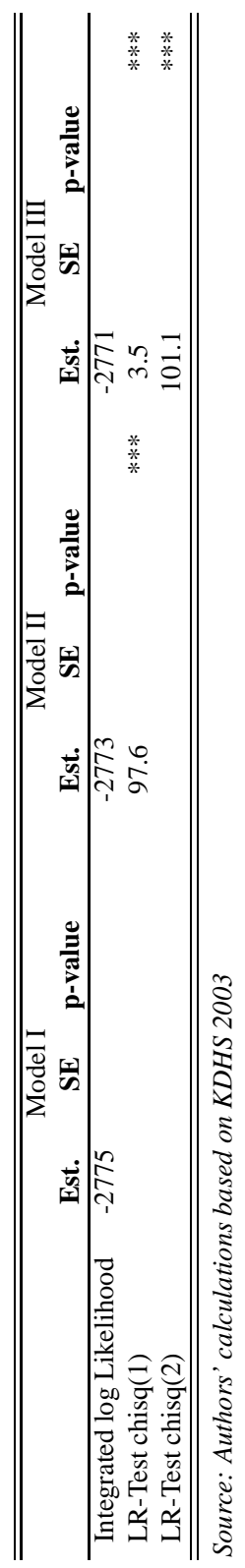

Johannes Gräb - 978-3-653-00480-9 
As expected, most of the variables fail in explaining wasting differentials. In our final model, Model III, coefficients of mother's educational status, the water supply index and the first child dummy have the expected sign but are slightly insignificant. Just four variables appear significant. Mother's BMI, itself an indicator of nutritional status, is positively affiliated with children's weight. The quality of the sanitation facility effects positively the weight-for-age status.

Once again, geographic and political factors seem to play a major role in determining nutritional and anthropometric outcomes. The lower a province's ratio of people per physician, i.e. the easier the access to medical care in that province, the higher the children's wasting score. The closer Lake Victoria, the higher average z-values of wasting. ${ }^{19}$

\section{Mortality}

Results of the under-5 mortality regressions are presented in Table 3.7. ${ }^{20}$ Once again, Model I is the standard single level regression model. Model II incorporates a household level random effect. In Model III a community effect is included in addition to the household effect. Regression results will again be reported based on the statistically superior model, Model III.

\footnotetext{
${ }^{19}$ As explained in section 3.4.1 we would expect the distance effect to vary continuously with respect to wasting outcomes. Robustness checks which are not reported here confirm that distance to Lake Victoria only seems to matter when captured continuously. In the full model the distance effect when included as a dummy variable always turned out to be statistically insignificant.

${ }^{20} \mathrm{Hazard}$ rates, the probability of death in $t$ conditional on survival up to $t$, are to be interpreted in relation to 1 . Thus, a hazard ratio of 1.2 implies a $20 \%$ higher risk of death. 


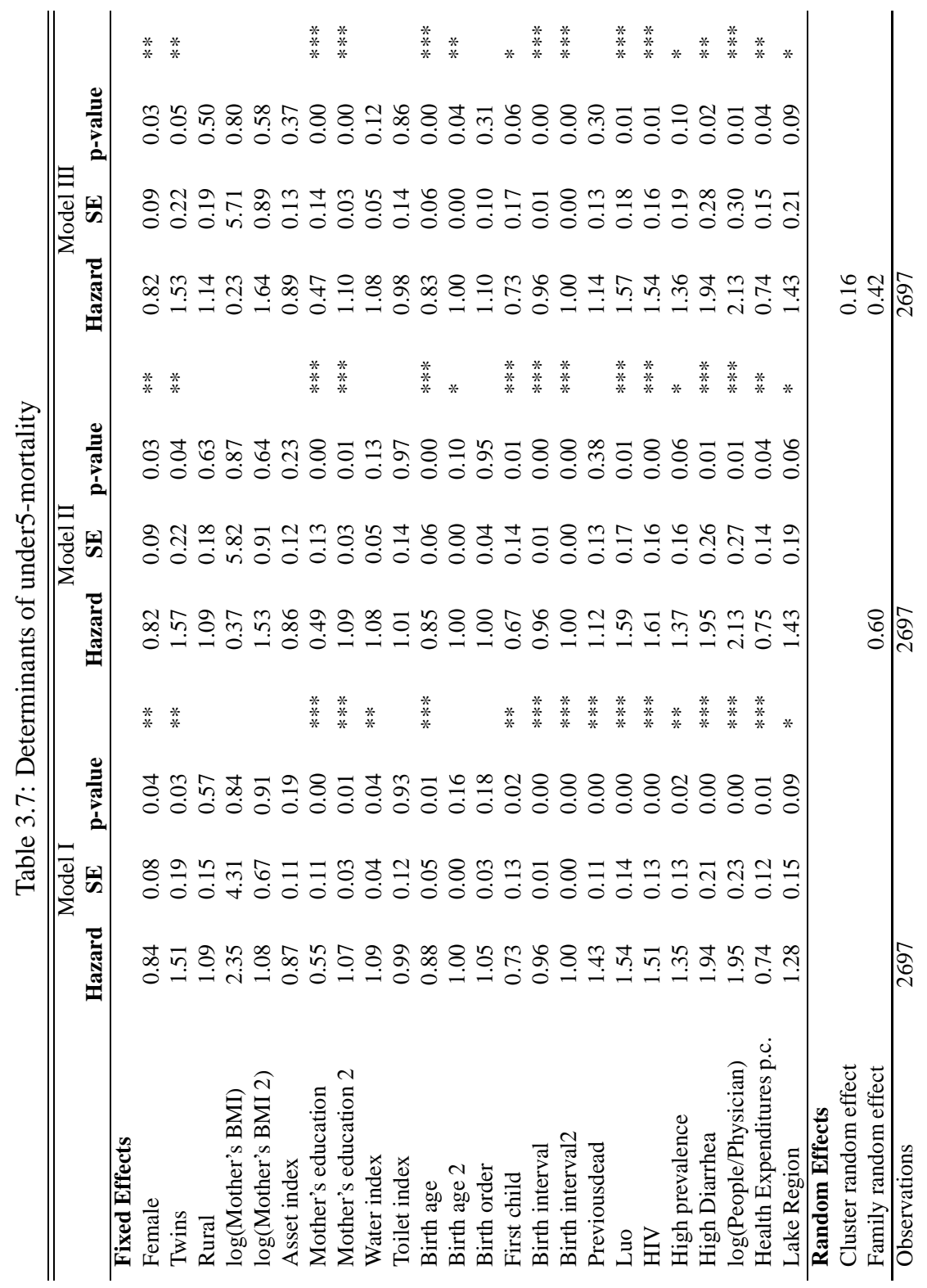




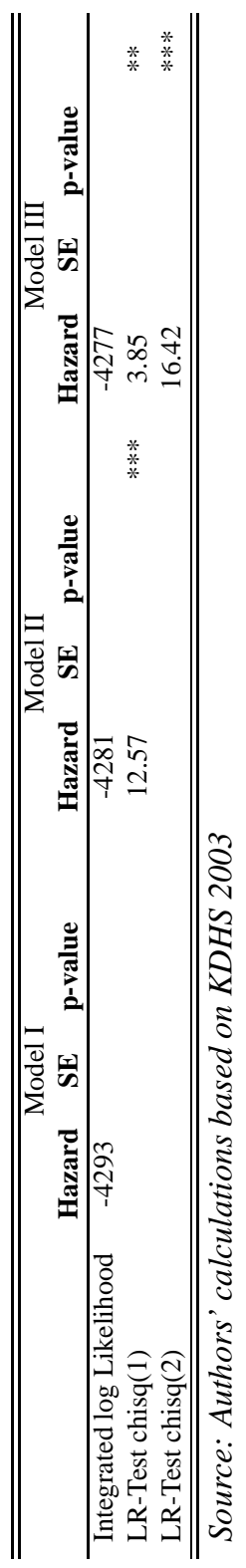

Johannes Gräb - 978-3-653-00480-9 
In Model III, coefficients on all variables that are commonly included in under5 mortality regressions show a reasonable economic size, take the expected signs and are in line with the empirical literature. The hazard rate is approximately $20 \%$ lower for girls than for boys. Being a twin increases the probability of death by $50 \%$. The location of the family, in terms of rural or urban, does not have a significant impact on survival chances. Interestingly, the same is true for the body mass index of the mother. Children stemming from better nourished mothers do not exhibit a lower risk of death. Children from richer households do, ceteris paribus, not seem to have a lower probability of death. ${ }^{21}$ The educational background of a mother evolves as highly significant, showing that children's survival chances are higher, the better educated the mother. Neither the quality of the water source nor the hygienic status of the household seems to have a considerable impact on under-5 mortality in Kenya. Moreover, a higher age of the mother at birth, a longer preceding birth interval and being the first born child in a family, increases a child's survival probability. Significant squared terms with reversed signs implicate diminishing returns of the factors.

Before examining the context specific determinants of under-5 mortality, it is useful to briefly discuss the benefits obtained from the adopted multilevel modeling strategy in this particular case. Comparing the highly significant family random effect of 0.60 in Model II with the family random effect of the unreported regression model without covariates but only the family random effect, of 0.90 , allows to conclude that the observed covariates were capable of explaining approximately $33 \%$ of the variation in child deaths across families. The family random effect of 0.60 is, however, likely to pick up unobserved heterogeneity on the community level; meaning that variation in child mortality across families could partly be explained by disparities in unobserved characteristics across communities. Indeed, the family random effect decreases by another $30 \%$ to 0.42 in Model III. The significant unobserved heterogeneity on the community level of 0.16 might be due to differences in unobserved child or family factors across communities. Since we included a large set of variables on both levels, we are, however, interpreting this effect as stemming from unobserved community, i.e. geographic or infrastructure, characteristics, that play a major role in explaining differences in mortality rates across Kenya. As expected, after the inclusion of family and community random effects standard errors of almost all covariates increase, lowering their statistical significance. Moreover, we do not only observe changes in the standard errors, but as well differences when comparing the hazard estimates of the models. Consistent with previous literature on multilevel frailty models (Omariba et al., 2007; Sastry, 1997a), effects of socioeconomic household level variables (assets, edu-

${ }^{21}$ This missing significance might to some extent result from the high correlation $(0.47)$ of the asset index with the educational status of the mother. 
cation) increase, while individual risk variables (birth order, previous child died) decrease. Especially striking is the decreasing effect of the variable that indicates whether the previously born child is still alive. This variable, highly correlated among siblings, is a clear indictor of families with higher mortality risk. The higher the unobserved frailty effect, the higher the probability that a sibling has already died. Neglecting the frailty leads to an overestimation of this effect. Contrary, factors independent of the unobserved frailty and among siblings, e.g the sex of the child, or the age of the mother, show only slight changes. ${ }^{22}$

Taking into account the specific cultural, geographic and political context we included variables on ethnicity, HIV-status, malaria and diarrhea prevalence, the Lake Victoria region, the number of people per physician and per capita health expenditures. Given the remarkable correlation of these factors, a simultaneous inclusion seems crucial to get unbiased estimates. All of these variables turn out to be significant.

Even after the inclusion of behavioral characteristics, being born to a Luo mother seems to have an adverse effect on survival chances. Controlling for geographical factors and the HIV-status, this result strongly points to an influence of adverse cultural practices (pre and post natal behavior), which were either unobserved or could not be considered in the regression. The potential influence of the latter has been illustrated in section 3.5.2 by the low share of Luo bearing their child in official health centers and the low vaccination rates of Luo's children signifying the awkward pre and post natal behavior of Luo. Omariba et al. (2007) undergo a similar study on child mortality in Kenya neglecting, however, HIV-infection and geographic factors. They conclude an excess risk of 10.5 for Luo children compared to children from Kikuyu ${ }^{23}$ mothers. Their much higher coefficient suggests an exaggeration of the Luo effect based on missing but highly correlated variables in their study.

The HIV status of the mother has severe negative impact on the survival status of a child. We estimate a hazard rate of around 1.5 bearing in mind that the coefficient is likely to be underestimated. ${ }^{24}$

Based on the results in Model III, the excess risk of living in a high risk malaria area is around $35 \%$ and therefore we confirm that malaria prevalence has a strong

\footnotetext{
${ }^{22}$ Underestimation of socioeconomic variables is a standard result in hazard models that are not controlling for unobserved heterogeneity. The mathematical foundation can be in found Guo and Rodriguez (1992)

${ }^{23}$ Living primarily in Nairobi, Central province and the Rift Valley, the Kikuyu represent the largest ethnic group in Kenya.

${ }^{24}$ In a longitudinal survey for rural Tanzania, Ng'weshemi et al. (2003) report a child death hazard ratio for maternal HIV infection of 2.3. Zaba et al. (2005) find in a cohort study for Uganda, Tanzania, and Malawi a hazard rate of 2.9. In a retrospective panel data study for Burkina Faso, Becher et al. (2004) estimate the hazard rate of mother's survival status to be 5.4 for children aged $1-5$.
} 
impact on mortality rates. Since to our knowledge no studies exist that tried to explicitly capture malaria prevalence as a covariate in a comparable setting we are not able to compare the magnitude of the coefficient to other studies directly. Nonetheless, recent studies from the medical literature (Snow et al., 1998; Omumbo et al., 2004; Ndugwa et al., 2008) commonly underscore the still massive impact of malaria on mortality in Kenya and Sub-Saharan Africa as well.

Besides malaria and HIV, diarrhea is presumed to be one of the most important drivers of child mortality. Our estimation suggests that living in a high risk diarrhea community almost doubles the excess risk of dying. This effect is possibly triggered by poor water quality. Even though we controlled for the type of water source, no direct indicator of water quality was available.

The negative effects of limited access to public funds are eminent. Children residing in provinces that receive lower public health expenditures and suffer under a higher share of people per physician exhibit significantly higher risk exposure. Political discrimination seems to be an important factor of the spatial variation in under-5 mortality rates.

Despite controlling for geography, ethnicity and political outcomes, we still obtain a positive and significant coefficient for the Lake Victoria region on under5 mortality. This demonstrates the unique misanthropic environment of the region, which could not be sufficiently captured by the variables at hand. ${ }^{25}$ The positive dummy might also pick up the natural malaria barrier of the East African rift valley - arising only some kilometers away from Lake Victoria - reducing the risk of malaria drastically.

The regression results confirm the theoretical considerations and descriptive findings of sections 3.4 and 3.5.2, respectively. The vast mortality rates of the Lake Victoria region rest upon a simultaneous impact of unfavorable geographic, cultural and political factors. There might be other regions in Sub Saharan Africa showing severe water pollution, elevated climate suitability for malaria transmission and high susceptibility for HIV infection. In the case of the Lake Victoria region of Kenya, these adverse geographic conditions are, however, found in a territory which is not only primarily populated by an ethnicity demonstrating adverse pre and post natal behavior but which is also suffering from political discrimination leading to underdeveloped access to health infrastructure.

\section{Family clustering decomposition}

The regression analyses conducted above provided insights on causal effects of potential individual malnutrition and mortality drivers. From a policy perspec-

\footnotetext{
${ }^{25}$ Recall, that the risk exposure to diarrhea and malaria was measured on the community and district level, respectively. Moreover, data on public health expenditures was available on the provincial level only.
} 
tive it is of interest to know those covariates that explain most of the variation in malnutrition and mortality outcomes across families or communities. Which covariates link children's risk of malnutrition or children's survival chances within a family or community? Gaining knowledge of these key variables allows policy makers to improve the targeting performance of poverty alleviation programmes.

An insight to this question can be derived by observing the variation of the family or community random effect (frailty) when each covariate is included exclusively in the null model or omitted exclusively from the full model. If the specific variable accounts for a large part of the overall variation, the variance of the random effect should decrease substantially when included in the null model and increase substantially when omitted from the full model. We will conduct this test for each variable for the stunting, wasting and mortality regression. To keep things clear we will limit this analysis for the clustering of malnutrition and mortality outcomes within families. Therefore we will use the Model II specification.

The results are shown in Table 3.8. The first line depicts the variation of the family random effect when no covariate is included (null model), and when all covariates are included (full model). The results indicate that the entire set of covariates accounts for $27 \%, 39 \%$ and $34 \%$ of the amount of family clustering, i.e. the between family variation, for the stunting, wasting and mortality regression, respectively.

Concerning the stunting regression, a mother's HIV and children's diarrhea status account for the largest proportion of the between family variation in stunting outcomes. This result holds for the inclusion of the variables in the null model and their exclusion from the full model. The same variables appear to be largely responsible for the family level clustering of wasting outcomes. The BMI of the mother emerges as another important factor for the between family variation in wasting rates. As for the regression results, a different pattern emerges when considering the under-5 mortality within family clustering. Mother's education, the survival status of the previous child and being the child of a Luo mother matters the most when explaining differences in mortality outcomes between families in Kenya.

These results should not be confounded with the regression results of Tables 3.5 - 3.7. For example, being a female or living around Lake Victoria has a significant impact on a child's nutritional and mortality outcome. Those variables are, however, better suited to explain the between sibling and between region variation respectively, than the between family variation. 


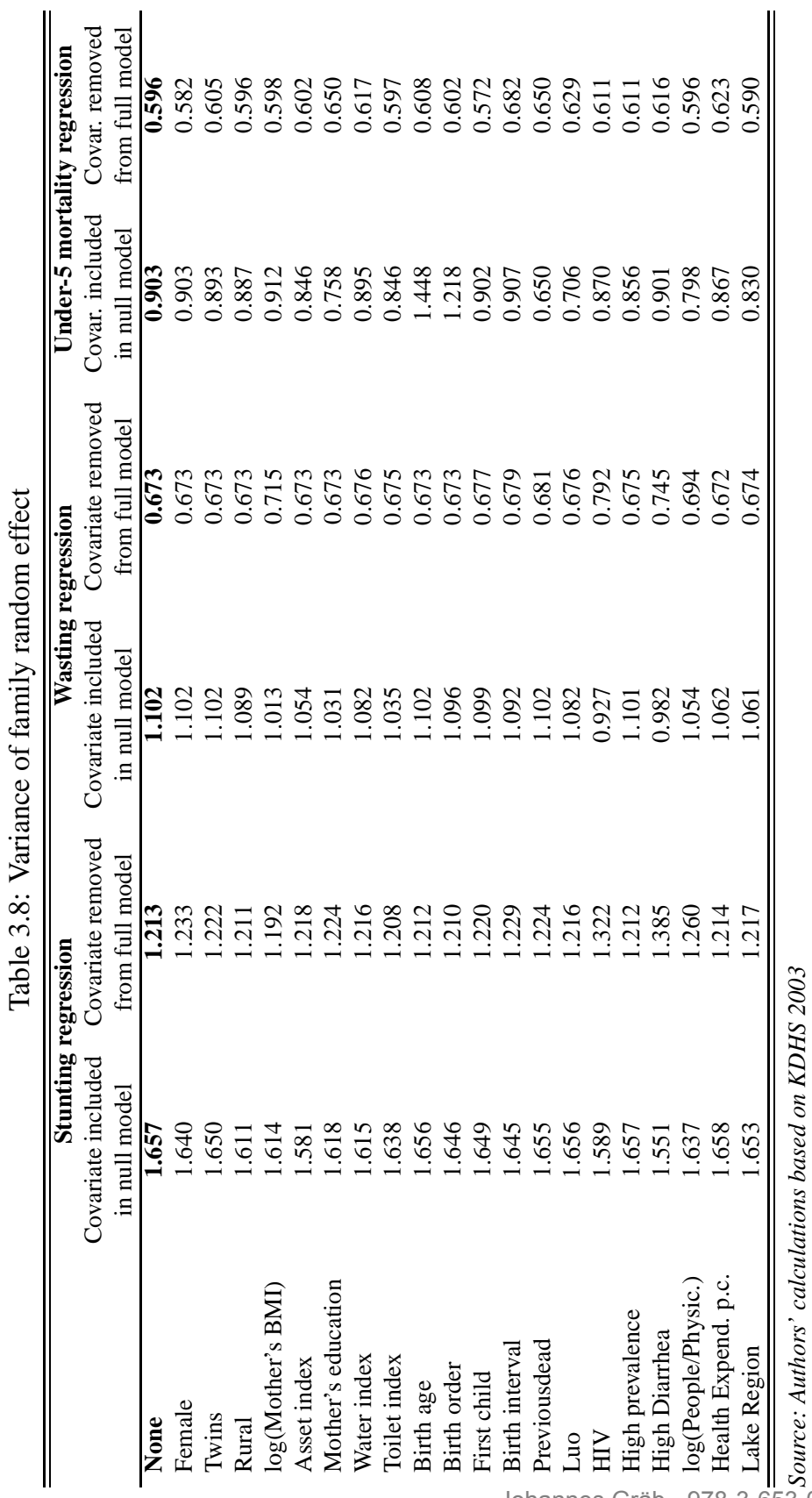




\subsection{Conclusion}

Kenya's Lake Victoria region is marked by an interesting puzzle. Under-5 mortality rates are by far the highest in the country, while at the same time anthropometric indicators of children show remarkable good values. The extent of this abnormity becomes even more astonishing when comparing the Lake Victoria area to other regions in Sub-Saharan Africa. Nowhere else in the whole of SSA we find such a strong disconnection of anthropometric and mortality outcomes.

In order to examine and understand the causes of this unusual phenomenon we undertake the uncommon step to analyze the determinants of mortality and undernutrition jointly. Moreover, to reduce the likelihood of obtaining biased and inefficient estimates in our multivariate regressions we construct a new set of context specific variables that supplements the conventional DHS data in addition to the application of suitable multilevel modeling techniques.

Our findings point to a unique interplay of cultural, geographical and political factors in the Lake Victoria region which are responsible for causing the described paradox. Concerning the under-5 mortality pattern in Kenya and around Lake Victoria we find that a salient disease environment characterized by extremely high malaria prevalence, polluted water sources and high rates of infectious diseases like HIV/AIDS is one of the key drivers of the massive under-5 mortality rates in the lake region. Furthermore, we see that even after controlling for mother's age at birth, birth spacing, birth order and HIV-status an ethnic specific effect remains. Being born to a Luo mother affects survival chances adversely, most likely based upon unfavorable unobserved pre and post natal behavior. Political discrimination does also seem to be an important factor of the spatial variation in under-5 mortality rates. Children residing in provinces that receive lower public health expenditures - such as Nyanza province - exhibit significantly higher risk exposure. In addition, the results indicate that even after inclusion of a rich set of covariates and controlling for clustering in unobserved characteristics, we are still confronted with an unusual high mortality rate in the Lake Victoria region that remains unexplained by the covariates and that is most likely to be attributed to insufficiently captured geographical and political factors.

A similar interplay of geographic conditions and cultural factors is found to constitute the extremely low incidence of stunting and wasting in the Lake Victoria region. While fish consumption in combination with an overall food secure situation spurs the growth process of children close to the lake and therefore leads to the much higher body height of children in the Lake Victoria area, the food security situation per se leads to ceteris paribus better wasting rates in the area.

Although these results are already very important for policy making we further examined which single factors contribute most to explaining differences in malnutrition and mortality between Kenyan families. Our analysis reveals that the Johannes Gräb - 978-3-653-00480-9 
hiv status of the mother and children's diarrhea status explain the largest part in the variation of stunting outcomes between families while the educational attainment of the mother, the survival status of the previous child and being a Luo turn out to be the most important sources in explaining mortality differentials between families.

Our findings demonstrate the relevance of considering and understanding the country specific context, when data on child mortality and malnutrition is analyzed. We do not challenge the epidemiological literature in the sense that we do not question that on the individual level a causal relationship between nutritional and mortality outcomes exists. The analysis raises a serious concern when using children's height status as a reliable proxy for health or income. This is only advisable when geographic, cultural and political contexts are comparable and this is often unlikely to be the case in cross-country or cross-regional analysis. 


\section{Appendix}

Table A.9: Regional Codes

\begin{tabular}{ll}
\hline \hline Code & Region \\
\hline 1 & Hauts Bassins \\
2 & Boucle de Mouhoun \\
3 & Sahel \\
4 & Est \\
5 & Sud-Ouest \\
6 & Centre Nord \\
7 & Centre Ouest \\
8 & Plateau Central \\
9 & Nord \\
10 & Centre Est \\
11 & Centre (Ouagadougou) \\
12 & Cascades \\
13 & Centre Sud \\
\hline \hline
\end{tabular}

Figure A.7: Map of Burkina Faso

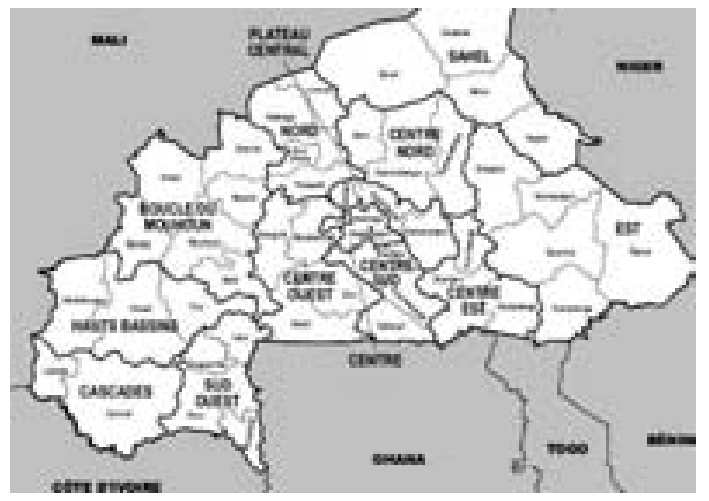

Source: ORC (2004) 
Figure A.8: BLUP of HH Size - 1994

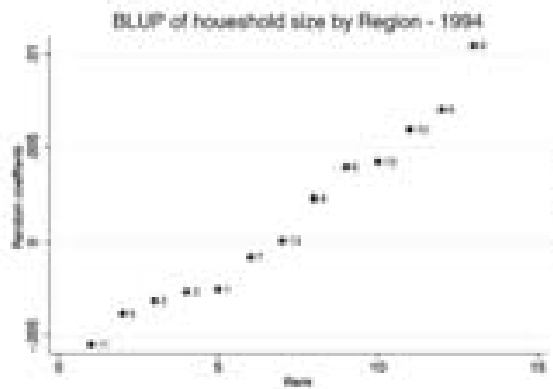

Figure A.10: BLUP of HH Size - 2003

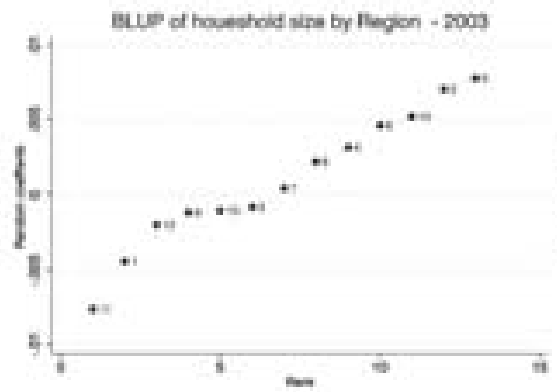

Figure A.12: Children per Adult - 1998

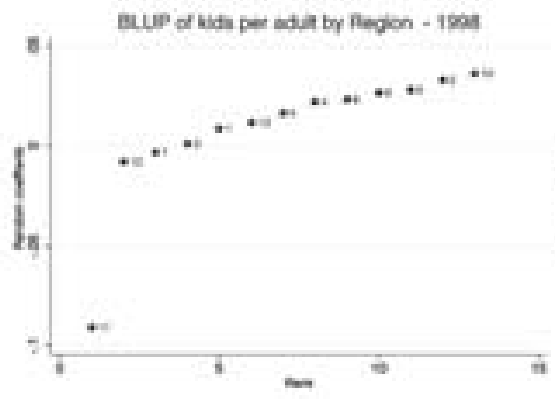

Figure A.9: BLUP of HH Size - 1998

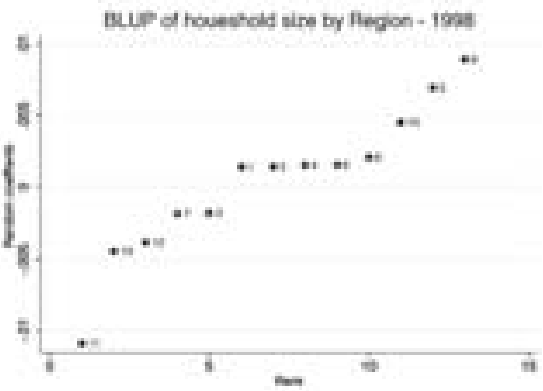

Figure A.11: Youth per Adult - 1994

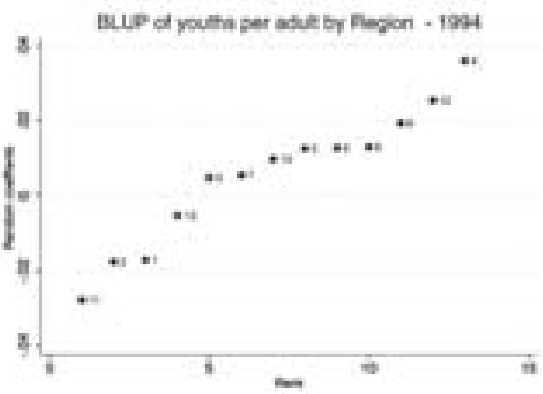

Figure A.13: Youth per Adult - 2003

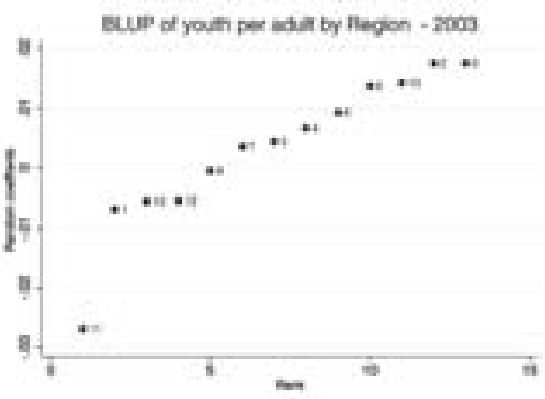


Figure A.14: Education - 1994

Figure A.15: Education - 1998
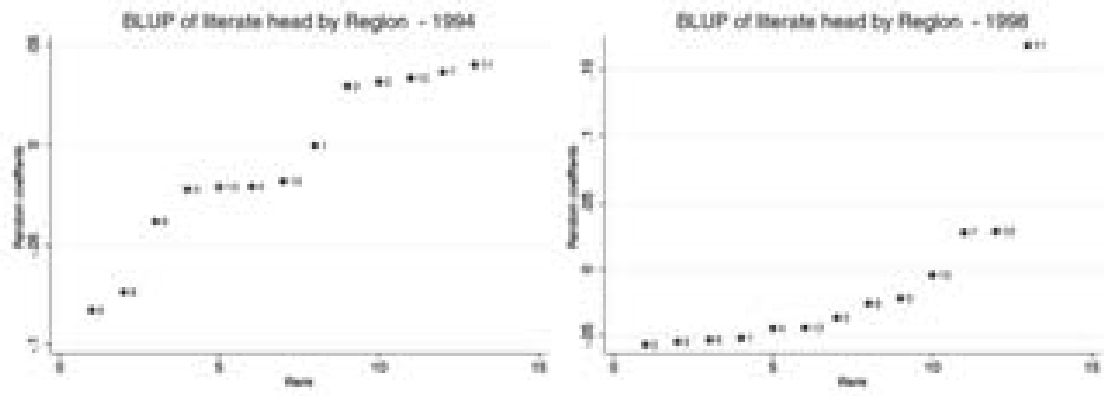

Figure A.16: Education - 2003

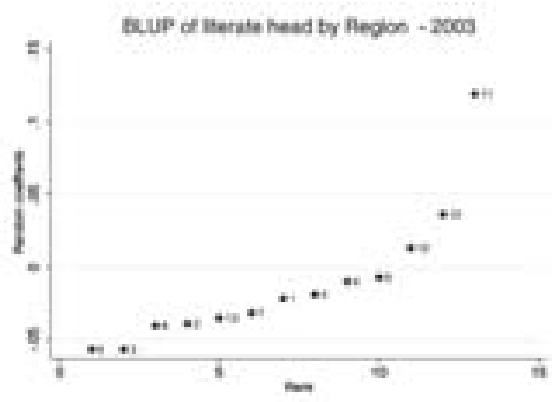


Johannes Gräb - 978-3-653-00480-9

Downloaded from PubFactory at 01/11/2019 02:31:01AM

via free access 


\section{Bibliography}

Aassve, Arnestein and Bruno Arpino, "Dynamic Multi-Level Analysis of Households' Living Standards and Poverty: Evidence from Vietnam," 2007. ISER working papers No 2007-10.

Acemoglu, Daron, Simon Johnson, and James A. Robinson, "The Colonial Origins of Comparative Development: An Empirical Investigation," American Economic Review, 2001, 91, 1369-1401.

Akin, Johan, David Guilkey, and Barry Popkin, "A child health production function estimated from longitudinal data," Journal of Development Economics, 1992, 38, 323-351.

Alwy, Alwiya and Susanne Schech, "Ethnic Inequalities in Education in Kenya," International Education Journal, 2004, 5 (2), 266-274.

Anderson, Carl A., David L. Duffy, Nicholas G. Martin, and Peter M. Visscher, "Estimation of Variance Components for Age at Menarche in Twin Families," Behavior Genetics, 2007, 37, 668-677.

Angeles, Gustavo and Stanislav Kolenikov, "The Use of Discrete Data in Principal Component Analysis: Theory, Simulations, and Application to Socioeconomic Indices," Proceedings of the American Statistical Association, 2004.

Atkinson, Anthony B., The Economics of Inequality, 2 ed., Oxford: Claredon Press, 1983.

_ , "On the Measurement of Poverty," Econometrica, July 1987, 55 (4), 749-764.

_ and Francois Bourguignon, "The Comparison of Multi-Dimensioned Distributions of Economic Status," Review of Economic Studies, April 1982, 49 (2), $183-201$.

Banerjee, Abhijit V., "New Development Economics and the Challenge to Theory," Economic Policy Weekly, December 2005, 40. 
Bardhan, Pranab, "Theory or Empirics in Development Economics," Economic Policy Weekly, December 2005, 40.

Basu, Kaushik, "The New Empirical Development Economics: Remarks on Its Philosophical Foundations," Economic Policy Weekly, December 2005, 40.

Becher, Heiko, Olaf Müller, Albrecht Jahn, Adjima Gbangou, Gisela KynastWolf, and Bocar Kouyaté, "Risk factors of infant and child mortality in rural Burkina Faso," Bulletin of the World Health Organization, April 2004, 82 (4), 265-273.

Behrman, Jere R. and Anil B. Deolalikar, "Health and Nutrition," in H. Chenery and T.N. Srinivasan, eds., Handbook of Development Economics No. 1, Elsevier 1988, pp. 631-711.

Béné, Christophe and Sonja Merten, "Women and Fish-for-Sex: Transactional Sex, HIV/AIDS and Gender in African Fisheries," World Development, 2008, 36 (5), 875-899.

Benson, Todd, Jordan Chamberlin, and Ingrid Rhinehart, "An investigation of the spatial determinants of the local prevalence of poverty in rural Malawi," Food Policy, 2005, 30 (5-6), 532-550.

Besley, Tim and Louise Cord, Delivering on the promise of pro-poor growth: Insights and lessons from country experiences, New York: Palgrave Macmillan, 2007.

Bigman, David, Stefan Dercon, Dominique Guillaume, and Michel Lambotte, "Community Targeting for Poverty Reduction in Burkina Faso," World Bank Economic Review, 2000, 14 (1), 167-193.

Bolstad, William M. and Samuel O. Manda, "Investigating Child Mortality in Malawi Using Family and Community Random Effects: A Bayesian Analysis," Journal of the American Statistical Association, March 2001, 96 (453), 12-19.

Borgoni, Riccardo, Ulf-Christian Ewert, and Alexia Fürnkranz-Prskawetz, "How important are household demographic characteristics to explain private car use patterns? A multilevel approach to Austrian data," MPIDR Working Papers WP-2002-006 Feb 2002.

Bossert, Walter, Conchita D'Ambrosio, and Vito Peragine, "Deprivation and Social Exclusion," Economica, November 2007, 74 (296), 777-803. 
_ , Satya R. Chakravarty, and Conchita D'Ambrosio, "Poverty and Time," 2008. ECINEQ, Society for the Study of Economic Inequality, Working Papers No.87.

Bound, John, Charles Brown, and Nancy Mathiowetz, "Measurement error in survey data," in J.J. Heckman and E. Learner, eds., Handbook of Econometrics, Vol. 5, Amsterdam: Elsevier Science, 2001, pp. 3705-3843.

Bourguignon, François and Satya R. Chakravarty, "Multidimensional poverty orderings," Delta Working Paper 2002-22, 2002.

_ and _ , "The measurement of multidimensional poverty," Journal of Economic Inequality, 2003, 1, 25-49.

Bozzoli, Carlos, Angus Deaton, and Climent Quintana-Domeque, "Adult height and childhood disease," Demography, forthcoming 2009.

Breen, Richard and Pasi Moisio, "Poverty dynamics corrected for measurement error," Journal of Economic Inequality, 2004, 2 (3), 171-191.

Cameron, A. Colin and Pravin K. Trivedi, Microeconometrics, Cambridge University Press, 2005.

Caulfield, Laura E., Stephanie A. Richard, and Robert E. Black, "Undernutrition as an Underlying Cause of Malaria Morbidity and Mortality in Children Less Than Five Years Old," The American Journal of Tropical Medicine and Hygiene, 2004, 71 (Suppl 2), 55-63.

CBS, MOH, and ORC Macro, Final Report on the Kenyan Demographic and Health Survey 20032004.

Chattopadhyay, Raghabendra and Esther Duflo, "Women as Policy Makers: Evidence from a Randomized Policy Experiment in India," Econometrica, 2004, 5, 1409-44.

Chesoni, Atsango, "Ethnic Chauvinism, Xenophobia and Gender Inequality in Kenya," In: Reading on Inequality in Kenya, vol.1, Society for International Development, 2006, pp. 195-252.

Chronic Poverty Research Center, The Chronic Poverty Report 2004-05 Institute for Development Policy and Management, University of Manchester 2004.

Cohen, John M., "Ethnicity, Foreign Aid, and Economic Growth in Sub-Saharan Africa: The Case of Kenya," Harvard - Institute for International Development Working Paper no. 520, 1995. 
Colony and Protectorate of Kenya, Annual Medical Report ending 31st Decmeber of the year 1922, 1923.

Cox, David R., "Regression models and life tables," Journal of the Royal Statistical Society Series B, 1972, 34, 187-220.

- and David Oakes, Analysis of Survival Data, New York: Chapman and Hall, 1984.

Cruces, Guillermo, "Income Fluctuations, Poverty and Well-Being Over Time: Theory and Application to Argentina," 2005. Discussion Paper No. DARP 76.

Cutler, David, Angus Deaton, and Adriana Lleras-Muney, "The determinants of Mortality," Journal of Economic Perspectives, 2006, 20 (3), 97-120.

De Vreyer, Philippe, Javier Herrera, and Sandrine Mesplé-Somps, "Consumption Growth and Spatial Poverty Traps: An Analysis of the Effect of Social Services and Community Infrastructures on Living Standards in Rural Peru," in F. Nowak-Lehmann and S. Klasen, eds., Poverty, Inequality, and Policy in Latin America, MIT Press, February 2009, pp. 129-156.

Deaton, Angus, "Height, health, and inequality: the distribution of adult height in India," American Economic Review, 2008, 98 (2), 468-474.

Dercon, Stefan, "Growth and Shocks: Evidence from rural Ethopia," Journal of Development Economics, 2004, 74 (2), 309-329.

DiPrete, Thomas A. and Jerry. D. Forristal, "Multilevel Model: Methods and Substance," Annual Review of Sociology, 1994, 20, 331-357.

Duclos, Jean-Yves, Abelkrim Araar, and John Giles, "Chronic and Transient Poverty: Measurement and Estimation, with Evidence from China," 2006. IZA Discussion Paper No. 2078, IZA, Bonn.

_ , David Sahn, and Stephen D. Younger, "Robust Multidimensional Poverty Comparisons," Economic Journal, 2006, 116 (514), 943-968.

_ , _ , and _ , "Robust Multidimensional Spatial Poverty Comparisons in Ghana, Madagascar, and Uganda," World Bank Economic Review, 2006, 20 (1), 91113.

Ecob, Russel, "A Multilevel Modelling Approach to Examining the Effects of Area of Residence on Health and Functioning," Journal of the Royal Statistical Society, 1996, 159 (1), 61-75. 
Elbers, Chris, Jean O. Lanjouw, and Peter Lanjouw, "Micro-level Estimation of Poverty and Inequality," Econometrica, 2003, 71, 355-364.

Fawzi, Wafaie W, M Guillerino Herrera, Donna L Spiegelman, Alawia El Amin, Penelope Nestel, and Kainal A Mohamed, "A prospective study of malnutrition in relation to child mortality in the Sudan," The American Journal of Clinical Nutrition, 1997, 65, 1062-9.

Fearn, H., An African Economy : A Study of the Economic Development of the Nyanza Province of Kenya, 1903-1953, Oxford University Press, 1961.

FEWS-Net, "Greater Horn of Africa Food Security Bulletin," Technical Report, FEWS-Net August 2004.

Filmer, Deon and Lant Pritchett, "Estimating Wealth Effects withour Expenditure Data - or Tears: An Application to Educational Enrollments in States of India," Demography, 2001, 38 (1), 115-132.

Fogel, Robert, "Economic Growth, Population Theory, and Physiology: The Bearing of Long-Term Processes on the Making of Economic Policy," American Economic Review, 1994, 84 (3), 369-395.

Foster, James E., "A Class of Chronic Poverty Measures,” 2007. Vanderbilt University WP No. 07-W01.

- and Anthony F. Shorrocks, "Inequality and Poverty Orderings," European Economic Review, 1988, 32, 654-662.

_ and _, "Poverty Orderings," Econometrica, 1988, 56, 173-177.

_ and _ , "Poverty Orderings and Welfare Dominance," Social Choice and Welfare, 1988, 5, 179-189.

- and Jim Jin, "Poverty orderings for the Dalton utility-gap measures," in S. Jenkins, A. Kapteyn, and B. van Praag, eds., The Distribution of Welfare and Household Production: International Perspectives, London: Cambridge University Press, 1996, pp. 268-285.

_, Joel Greer, and Erik Thorbecke, "A Class of Decomposable Poverty Measures," Econometrica, 1984, 52, 761-776.

Frisch, Ragnar, "Editorial," Econometrica, 1933, 1, 1-4.

Gallup, John L., Jeffrey D. Sachs, and Andrew D. Mellinger, "Geography and Economic Development," 1998. NBER Working Paper 6849, NBER Cambridge, MA. 
Geheb, Kim, Sarah Kallocha, Modesta Medardb, Anne-Therese Nyapendic, Carolyne Lwenyac, and Mercy Kyangwad, "Nile perch and the hungry of Lake Victoria: Gender, status and food in an East African fishery," Food Policy, 2008, 33 (1), 85-98.

Goldstein, Harvey, Multilevel Statistical Models, Vol. 3, London: Edward Arnold, 2003.

Grimm, Michael, "Removing the anonymity axiom in assessing pro-poor growth," Journal of Economic Inequality, 2007, 5 (2), 179-197.

- and Isabel Günther, "Growth and Poverty in Burkina Faso. A Reassessment of the Paradox," Journal of African Economies, 2007, 16, 70-101.

- and Stephan Klasen, "Geography vs. Institutions at the Village Level," Center for European, Governance and Economic Development Research (cege) Discussion Papers 70, University of Goettingen Feb 2008.

_ , Andy McKay, and Stephan Klasen, Determinants of Pro-Poor Growth: Analytical Issues and Findings from Country Cases, London: Palgrave-Macmillan, 2007.

Grootaert, Christiaan and Ravi Kanbur, "The Lucky Few amidst Economic Decline: Distributional Change in Côte d'Ivoire as seen through Panel Datasets, 1985-1988," Journal of Development Studies, 1995, 31 (4), 603-619.

Guo, Guang and German Rodriguez, "Estimating a multivariate proportional hazards model for clustered data using the EM algorithm, with an application to child survival in Guatemala," Journal of the American Statistical Association, 1992, 87 (420), 969-976.

- and Hongxin Zhao, "Multilevel Modeling For Binary Data," Annual Review of Sociology, 2000, 26, 441-62.

Hall, Rober I. and Charles I. Jones, "Why do some countries produce so much more output than others?," Quarterly Journal of Economics, 1999, 114, 83-116.

Harttgen, Kenneth and Mark Misselhorn, "A Multilevel Approach to Explain Child Mortality and Undernutrition in South Asia and Sub-Saharan Africa," Ibero-America Institute Working Paper No. 152, 2006.

Hendry, David, "Econometrics: Alchemy or Science?," Economica, November 1980, 47, 387-406. 
Heston, Alan, Robert Summers, and Bettina Aten, "Penn World Table Version 6.1," 2002. Center for International Comparisons at the University of Pennsylvania (CICUP).

Hill, Kenneth, Boaz Cheluget, Siân Curtis, George Bicego, and Mary Mahy, "HIV and Increases in Childhood Mortality in Kenya in the Late 1980s to the Mid-1990s," 2004.

Hox, Joop J., Applied Multilevel Analysis, Amsterdam: TT-Publikaties, 1995.

Hoy, Michael and Buhong Zheng, "Measuring Lifetime Poverty," 2007. University of Colorado WP No. 07-01.

Hulme, David and Andrew Shepherd, "Conceptualizing Chronic Poverty," World Development, 2003, 31 (3), 403-424.

- and Andy McKay, "Identifying and Measuring Chronic Poverty: Beyond Monetary Measures," 2005. Mimeo, Institute for Development Policy and Management, University of Manchester.

Jacoby, Hanan G., "Access to Markets and the Benefits of Rural Roads," The Economic Journal, July 2000, 110, 713-737.

Jalan, Jyotsna and Martin Ravallion, "Transient Poverty in Postreform Rural China," Journal of Comparative Economics, 1998, 26, 338-357.

_ and _ , "Geographic Poverty Traps? A Micro Model of Consumption Growth in Rural China," Journal of Applied Econometrics, 2002, 17 (4), 329-346.

Jiang, Jiming and P. Lahiri, "Mixed Model Prediction and Small Area Estimation," Test, 2006, 15 (1), 1-96.

Kanbur, Ravi, “Goldilocks Development Economics," Economic Policy Weekly, December 2005, 40.

Kenya National Bureau of Statistics, "Basic Report on Well-being in Kenya," Technical Report 2006.

Keynes, John M., "Professor Tinbergen's Method,” Economic Journal, 1939, 49 (195), 558-577.

Klasen, Stephan, "Nutrition, Health, and Mortality in Sub Saharan Africa: Is there a Gender Bias?," Journal of Development Studies, 1996, 32 (6), 913-932. 
_ , "Poverty, undernutrition, and child mortality: Some inter-regional puzzles and their implications for research and policy," Journal of Economic Inequality, 2008, 6 (1), 89-115.

Leibenstein, Harvey, Economic Backwardness and Economic Growth, New York: Wiley, 1957.

Lombardía, María J. and Stefan Sperlich, "Multi-level Regression Between Fixed Effects and Mixed Effects Models," 2007. Center for Statistics WP ZfS14.2007.

MARA, “Mapping Malaria Risk in Africa,” Technical Report, MARA 2004.

Marques, Rejane C., Jos Garrofe Drea, Jos V. E. Bernardi, Wanderley R. Bastos, and Olaf Malm, "Maternal fish consumption in the nutrition transition of the Amazon Basin: Growth of exclusively breastfed infants during the first 5 years," Annals of Human Biology, 2008, 35 (4), 363-377.

Merlo, Juan, Basile Chaix, Min Yang, John Lynch, and Lennart Rastam, "A brief conceptual tutorial on multilevel analysis in social epidemiology: interpreting neighbourhood differences and the effect of neighbourhood characteristics on individual health," Journal Epidemiol. Community Health, 2005, 59, $1022-1029$.

${ }_{-},,_{-},,_{-}$, and _ , "A brief conceptual tutorial on multilevel analysis in social epidemiology: investigating contextual phenomena in different groups of people," Journal Epidemiol. Community Health, 2005, 59, 729-736.

Miguel, Edward, "Tribe or Nation? Nation Building and Public Goods in Kenya versus Tanzania," World Politics, 2004, 56, 327-362.

- and Mary Kay Gugerty, "Ethnic diversity, social sanctions, and public goods in Kenya," Journal of Public Economics, 2005, 89 (11-12), 2325-2368.

- and Michael Kremer, "Worms: Identifying Impacts on Education and Health in the Presence of Treatment Externalities," Econometrica, January 2004, 72 (1), 159-217.

Mookherjee, Dilip, "Is there too little theory in development economics today?," Economic Policy Weekly, December 2005, 40, 4328-4333.

Moradi, Alexander, "Towards an Objective Account of Nutrition and Health in Colonial Kenya: A Study of Stature in African Army Recruits and Civilians, 1880-1980," Journal of Economic History, forthcoming 2009. 
Mosley, W. Henry and Lincoln C. Chen, "An Anyalytical Framework for the Study of Child Survival in Developing Countries," Population and Development Review, 1984, 10, 25-45.

Muhula, Raymond, "Horizontal Inequality and Ethno-Regional Politics in Kenya," Unpublished Working Paper, 2008.

Nandy, Shailen, Michelle Irving, David Gordon, S.V. Subramanian, and George Davey Smith, "Poverty, child undernutrition and morbidity: new evidence from India," Bulletin of the World Health Organization, March 2005, 83 (3).

Ndugwa, Robert P, Heribert Ramroth, Olaf Müller, Momodou Jasseh, Ali Sié, Bocar Kouyaté, Brian Greenwood, and Heiko Becher, "Comparison of all-cause and malaria-specific mortality from two West African countries with different malaria transmission patterns," Malaria Journal, 2008, 71 (15).

Ng'weshemi, Japheth, Mark Urassa, Raphael Isingo, Gabriel Mwaluko, Juliana Ngalula, Ties Boerma, Milly Marston, and Basia Zaba, "HIV Impact on Mother and Child Mortality in Rural Tanzania," Journal of Acquired Immune Deficiency Syndromes, 2003, 33, 393-404.

Nyanjom, Othieno, "Inequality in Kenya's Health Sector," In: Reading on Inequality in Kenya, vol.1, Society for international development, 2006, pp. 97 156.

Ochumba, Peter B. O. and David I. Kibaara, "Observations on blue-green algal blooms in the open waters of Lake Victoria," African Journal of Ecology, 1989, $27,23-34$.

Oguttu, Hannington W., Fred W. B. Bugenyi, Heinz Leuenberger, Markus Wolf, and Reinhard Bachofen, "Pollution menacing Lake Victoria : Quantification of point sources around Jinja Town, Uganda," Water SA, 2008, 34 (1), 89-98.

Omariba, D. Walter Rasugu, Roderic Beaujot, and Fernando Rajulton, "Determinants of infant and child mortality in Kenya: an analysis controlling for frailty effects," Population Res. Policy Review, 2007, 26, 299-321.

Omumbo, Judith A, Simon I Hay, Carlos A Guerra, and Robert W Snow, "The relationship between the Plasmodium falciparum parasite ratio in childhood and climate estimates of malaria transmission in Kenya," Malaria Journal, June 2004, 3. 
Omwega, Reuben N., Richard Abila, and Caroyline Lwenya, "Fishing and poverty levels around Lake Victoria (Kenya)," Kenya Marine and Fisheries Research Institute Working Paper, 2003, 10, 25-45.

Oniang'o, R. K. and A. Komokoti, "Food Habits in Kenya: The Effects of Change and Attendant Methodological Problems," Appetite, 1999, 32, 93-96.

ORC Macro International Inc., Burkina Faso Enquête Démographique et de Santé. Rapport de synthèse 2004.

Oster, Emily, "HIV and Sexual Behavior Change: Why not Africa ?," NBER Working Paper No. 13049, 2007.

_ , "Routes of Infection: Exports and HIV Incidence in Sub-Saharan Africa," NBER Working Paper No. 13610, 2008.

Pankratz, V. Shane, Mariza de Andrade, and Terry M. Therneau, "RandomEffects Cox Proportional Hazards Model: General Variance Components Methods for Time-to-Event Data," Genetic Epidemiology, 2005, 28, 97-109.

Pelletier, D.L., E.A. Frongillo, D.G. Schroeder, and J.-H-Habicht, "The Effects of Malnutrition on Child Mortality in Developing Countries," Bulletin of the World Health Organization, 1995, 73 (4), 443-448.

Peters, Caroline and Rudo Niemeijer, "Protein-Energy Malnutrition and the Home Environment: A study among children in Coast Province, Kenya," University of Leiden - African Studies Centre Report no. 22/1987, 1987.

Ramalingaswami, Vulimiri, Urban Jonsson, and Jon Rode, "The Asian enigma," in Peter Adamson, ed., The Progress of Nations, 1996: The Nations of the World Ranked According to Their Achievements in Child Health, Nutrition, Education, Family Planning, and Progress for Women., 1996.

Ravallion, Martin and Quentin Wodon, "Poor Areas, or Only Poor People?," Journal of Regional Science, November 1999, 39 (4), 689-711.

_ , Shaohua Chen, and Prem Sangraula, "Dollar a day revisited," Policy Research Working Paper Series 4620, The World Bank May 2008.

Ray, Debray, "Introduction to development theory," Journal of Economic Theory, 2007, 137 (1), 1-10.

Rosenstein-Rodan, Paul, "Problems of industrialization of eastern and southeastern Europe," Economic Journal, 1943, 53, 202-211. 
Rosenzweig, Mark R. and Kenneth I. Wolpin, "Heterogeneity, Intrafamily Distribution, and Child Health," The Journal of Human Resources, 1988, 13 (4), 437-461.

Sachs, Jeffrey and Pia Malaney, "The Economic and Social Burden of Malaria," Nature, 2002, 415, 680-685.

Sahn, David E. and David Stifel, "Exploring Alternative Measures of Welfare in the Absence of Expenditure Data," Review of Income and Wealth, 2003, 49 (4), 463-489.

Sala-i-Martin, Xavier X., "The classical approach to convergence analysis," Economic Journal, 1996, 106, 1019-1036.

Sastry, Narayan, "Family-level clustering of childhood mortality risk in Northeast Brazil,” Population Studies, 1997, 51, 245-261.

_ , "A nested frailty model for survival data, with an application to the study of child survival in Northeast Brazi," Journal of the American Statistical Association, 1997, 92 (438), 426-435.

Scheren, P. A. G. M., H. A. Zanting, and A. M. C. Lemmens, "Estimation of water pollution sources in Lake Victoria, East Africa: Application and elaboration of the rapid assessment methodology," Journal of environmental management, 2000, 58 (4), 235-248.

Sen, Amartya, On Economic Inequality, Oxford: Clarendon Press, 1973.

_, "Poverty: an ordinal approach to measurement," Econometrica, 1976, 44, 219-231.

_ , Standard of Living, New York: Cambridge University Press, 1987.

Smith, Lisa C. and Lawrence Haddad, "How Potent Is Economic Growth in Reducing Undernutrition? What Are the Pathways of Impact? New Cross-Country Evidence," Economic Development and Cultural Change, October 2002, 51 (1), $55-76$.

Snow, Russel W., Eleanor Gouws, J. Omumbo, B. Rapuoda, M. H. Craig, and J. Ouma, "Models to predict the intensity of Plasmodium falciparum transmission: applications to the burden of disease in Kenya," Transactions of the Royal Society of Tropical Medicine and Hygiene, 1998, 92, 601-606.

Stata, Stata statistical software: Release 10.0 Stata Corp. 2007. 
Steckel, Richard, "Stature and the Standard of Living," Journal of Economic Literature, 1995, 3 (4), 1903-1940.

Strauss, John and Duncan Thomas, "Health, Nutrition, and Economic Development," Journal of Economic Literature, June 1998, 36 (2), 766-817.

_, Kathleen Beegle, Bondan Sikoki, Agus Dwiyanto, Yulia Herawati, and Firman Witoelar, "The Third Wave of the Indonesia Family Life Survey (IFLS3): Overview and Field Report," 2004. WR-144/1-NIA/NCHID, RAND Corporation.

Svedberg, Peter, "Undernutrition in Sub-Saharan Africa: Is there a Gender Bias?," Journal of Development Studies, 1990, 26 (3), 469-486.

-, Poverty and Undernutrition: Theory, Measurement and Policy, Oxford: Clarendon Press, 2000.

Therneau, Terry M., On mixed-effect Cox models, sparse matrices, and modeling data from large pedigrees. 2006.

Trussel, James and Germán Rodriguez, "Heterogeneity in demographic research," in Julian Adams, David A. Lam, Albert I. Hermanlin, and Peter E. Smouse, eds., Convergent Issues in Genetics and Demography, London: Oxford University Press, 1990, pp. 111-132.

Tseloni, Andromachi, "Multilevel modelling of the number of property crimes: household and area effects," Journal of the Royal Statistical Society A, 2006, 169 (2), 205-233.

UNDP, Human Development Report 2007/2008 UNDP 2007.

UNICEF, "The State of the World's Children," United Nations Children's Fund, 2008.

Vaupel, James W., Kindred lifetimes: frailty models in population genetics, New York: Oxford University Press,

_ , "Relatives' risks: frailty models of life history data," Theoretical Population Biology, 1990, 37 (1), 220-234.

Villamor, Eduardo, Lara Misegades, Maulidi R Fataki, Roger L Mbise, and Wafaie W Fawzi, "Child mortality in relation to HIV infection, nutritional status, and socio-economic background," International Journal of Epidemiology, January 2005. 
Wainaina, Gituro, "An Inequality Perspective of Education Structure and Performance in Kenya," In: Reading on Inequality in Kenya, vol.1, Society for international development, 2006, pp. 157-194.

Wamani, Henry, Anne Nordreghaug, Stefan Peterson, James K. Tumwine, and Thorkild Tyllesk, "Boys are more stunted than girls in Sub-Saharan Africa: A meta-analysis of 16 demographic and health surveys," BMC Pediatrics, 2007, 7 (17).

Watts, Harold W., "An Economic Definition of Poverty," in D.P. Moynihan, ed., On Understanding Poverty, New York: Basic Books, 1968.

Weinreb, Alexander A., "First Politics, Then Culture: Accounting for Ethnic Differences in Demographic Behaviour in Kenya," Population and Development Review, September 2001, 27 (3), 437-467.

Whitworth, Alison and Rob Stephenson, "Birth spacing, sibling rivalry and child mortality in India," Social Science and Medicine, 2002, 55, 2107-2119.

WHO, "WHO Child Growth Standards," WHO - Backgrounder2, 2006.

Williamson, Jeffrey G., Coping with city growth during the British Industrial Revolution, Cambridge; New York and Melbourne: Cambridge University Press, 1990.

Zaba, Basia, Jimmy Whitworth, Milly Marston, Jessica Nakiyingi, Anthony Ruberantwari, Mark Urassa, Raphaeli Issingo, Gabriel Mwaluko, Sian Floyd, Andrew Nyondo, and Amelia Crampin, "HIV and Mortality of Mothers and Children - Evidence From Cohort Studies in Uganda, Tanzania, and Malawi," Epidemiology, 2005, 16, 275-280.

Zheng, Buhong, "Aggregate Poverty Measures," Journal of Economic Surveys, 1997, 11, 123-163.

_ , "Poverty Orderings," Journal of Economic Surveys, 2000, 14 (4), 427-466. 


\title{
Göttinger Studien zur Entwicklungsökonomik Göttingen Studies in Development Economics
}

\author{
Herausgegeben von/Edited by Hermann Sautter
} und/and Stephan Klasen

Die Bände 1-8 sind über die Vervuert Verlagsgesellschaft (Frankfurt/M.) zu beziehen.

Bd./Vol. 9 Hermann Sautter / Rolf Schinke (eds.): Social Justice in a Market Economy. 2001.

Bd./Nol.10 Philipp Albert Theodor Kircher: Poverty Reduction Strategies. A comparative study applied to empirical research. 2002.

Bd./Vol.11 Matthias Blum: Weltmarktintegration, Wachstum und Innovationsverhalten in Schwellen ländern. Eine theoretische Diskussion mit einer Fallstudie über „Argentinien 1990-1999". 2003.

Bd./Vol.12 Jan Müller-Scheeßel: Die Privatisierung und Regulierung des Wassersektors. Das Beispiel Buenos Aires/Argentinien. 2003.

Bd./Vol.13 Ludger J. Löning: Economic Growth, Biodiversity Conservation, and the Formation of Human Capital in a Developing Country. 2004.

Bd./Nol.14 Silke Woltermann: Transitions in Segmented Labor Markets. The Case of Brazil. 2004.

Bd./Vol.15 Jörg Stosberg: Political Risk and the Institutional Environment for Foreign Direct Investment in Latin America. An Empirical Analysis with a Case Study on Mexico. 2005.

Bd./Vol.16 Derk Bienen: Die politische Ökonomie von Arbeitsmarktreformen in Argentinien. 2005.

Bd./Vol.17 Dierk Herzer: Exportdiversifizierung und Wirtschaftswachstum. Das Fallbeispiel Chile. 2006.

Bd./Vol.18 Jann Lay: Poverty and Distributional Impact of Economic Policies and External Shocks. Three Case Studies from Latin America Combining Macro and Micro Approaches. 2007.

Bd./Vol.19 Kenneth Harttgen: Empirical Analysis of Determinants, Distribution and Dynamics of Poverty. 2007.

Bd./Vol. 20 Stephan Klasen / Felicitas Nowak-Lehmann: Poverty, Inequality and Migration in Latin America. 2008.

Bd./Vol. 21 Isabel Günther: Empirical Analysis of Poverty Dynamics. With Case Studies from Sub-Saharan Africa. 2007.

Bd./Vol.22 Peter Dung: Malaysia und Indonesien: Wirtschaftliche Entwicklungsstrategien in zwei Vielvölkerstaaten. 2008.

Bd./Vol. 23 Thomas Otter: Poverty, Income Growth and Inequality in Paraguay During the 1990s. Spatial Aspects, Growth Determinants and Inequality Decomposition. 2008.

Bd./Vol. 24 Mark Misselhorn: Measurement of Poverty, Undernutrition and Child Mortality. 2008.

Bd./Vol. 25 Julian Weisbrod: Growth, Poverty and Inequality Dynamics. Four Empirical Essays at the Macro and Micro Level. 2008.

Bd./Vol. 26 Johannes Gräb: Econometric Analysis in Poverty Research. With Case Studies from Developing Countries. 2009.

www.peterlang.de 


\section{Three Essays on the}

\section{Econometric Analysis in}

\section{Poverty Research}

Dissertation

zur Erlangung des wirtschaftswissenschaftlichen Doktorgrades der Wirtschaftswissenschaftlichen Fakultät der Universität Göttingen

vorgelegt am 17.022009

von Johannes Gräb

aus Göttingen 
Erstgutachter: Prof. Michael Grimm

Zweitgutachter: Prof. Stephan Klasen, Ph.D.

Datum der Disputation: 24.04.2009 